Portland State University

PDXScholar

Fall 12-5-2016

\title{
Vulnerability and Protective Factors of Stress- Related Drinking: an Exploration of Individual and Day-Level Predictors of Alcohol Involvement
}

Cameron Trim McCabe

Portland State University

Follow this and additional works at: https://pdxscholar.library.pdx.edu/open_access_etds

Part of the Psychology Commons, and the Social Psychology and Interaction Commons Let us know how access to this document benefits you.

\section{Recommended Citation}

McCabe, Cameron Trim, "Vulnerability and Protective Factors of Stress-Related Drinking: an Exploration of Individual and Day-Level Predictors of Alcohol Involvement" (2016). Dissertations and Theses. Paper 3287.

https://doi.org/10.15760/etd.3278

This Dissertation is brought to you for free and open access. It has been accepted for inclusion in Dissertations and Theses by an authorized administrator of PDXScholar. Please contact us if we can make this document more accessible: pdxscholar@pdx.edu. 
Vulnerability and Protective Factors of Stress-Related Drinking:

An Exploration of Individual and Day-Level Predictors of Alcohol Involvement

by

Cameron Trim McCabe

A dissertation submitted in partial fulfillment of the requirements for the degree of

Doctor of Philosophy

in

Applied Psychology

Dissertation Committee:

Cynthia D. Mohr, Chair

Matthew Carlson

Leslie Hammer

Greg Townley

Portland State University

2016 
(C) 2016 Cameron Trim McCabe 


\begin{abstract}
Problem alcohol use has far-reaching economic, intra-, and interpersonal consequences. One particularly hazardous form of drinking pertains to the consumption of alcohol as a means of regulating stress, or drinking to cope. As such, it is critical to identify pathways through which stress-related alcohol use occurs, as well as protective factors which may mitigate the aforementioned consequences. To achieve this, I conducted three studies examining these topics at multiple levels of analysis among two at risk populations for engaging in problematic drinking: College students and military service members. Study 1 is a published manuscript examining the association between personality, a known vulnerability factor, and daily alcohol use among college students. This study tested whether these associations were mediated by the utilization of daily coping behaviors. Study 2 is an exploration of the association between of post-traumatic stress symptoms (PTSS) and alcohol involvement among employed service members. I conducted conditional process analysis to determine whether the indirect association of PTSS on alcohol involvement through coping motivations was conditional on one's perceived level of social support. Finally, Study 3 examined how daily experiences of occupational stressors influence alcohol consumption using a subsample of married and cohabiting participants from Study 2. I tested the moderating roles of coping motives and more adaptive, support-based coping strategies on work stress-daily drinking associations. Together, these studies help elucidate why individuals typically drink when stressed, who may be more apt to do so, and under what conditions these effects hold true.
\end{abstract}




\section{ACKNOWLEDGEMENTS}

I would first like to thank my advisor, Dr. Cynthia Mohr, for inviting me into her lab, setting me up for success, and cultivating high standards and expectations within all her students. To my committee for shaping this document into what it is, providing encouragement, and instilling the confidence to aim high. To the SERVe team who has inspired me with their passion, drive, and what they've accomplished. I am honored to say I have contributed to your efforts. To my "coheart" and lab mates for the ups and downs, laughter and anxiety, successes and struggles, enlightening conversations and utterly absurd ones.

To my family for encouraging me to set goals and stick with them, and for understanding when I forget to return calls.

To my wife who has supported me fully for the past 11 years. You've selflessly postponed your own plans as I pursued this goal, and endured moves up and down this coast. I can never repay your patience, love, and support, but will continue to make the effort every day. I couldn't have made it here without you.

To my daughter, you are my everything. You provide clarity and perspective on good days and bad. Your smile, laughter, and penchant for sarcasm at such a young age fill my heart. I cannot wait for the great adventures to come.

Finally, to the men, women, and families of the U.S. Armed Forces, I thank you for your service and sacrifice. I entered this field hoping to improve the lives of our warfighters, and hope my efforts will one day contribute in some measure. 


\section{Table of Contents}

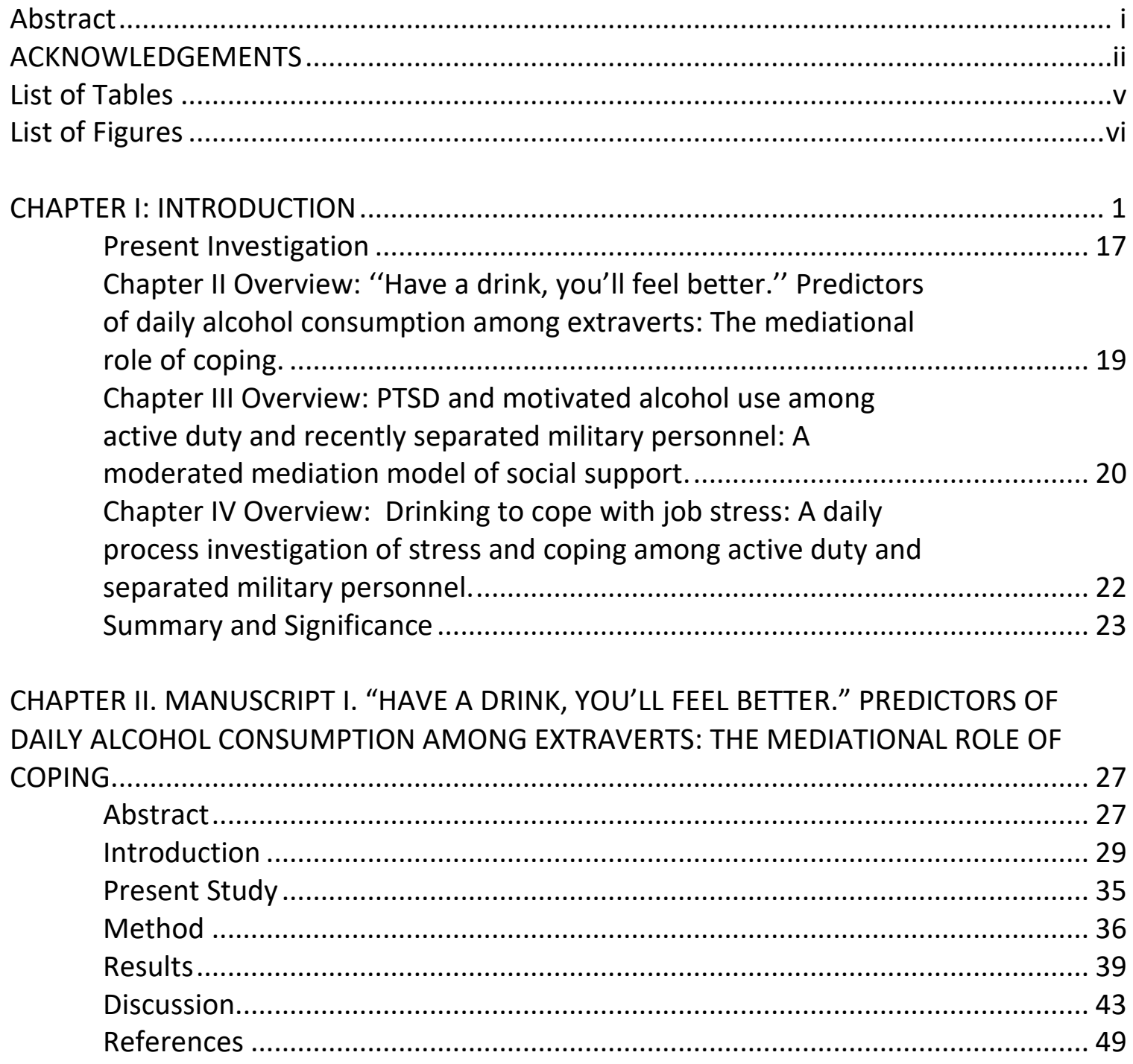

CHAPTER III. MANUSCRIPT II. PTSD SYMPTOMOLOGY AND MOTIVATED ALCOHOL USE AMONG MILITARY SERVICE MEMBERS: A CONDITIONAL INDIRECT EFFECT MODEL OF

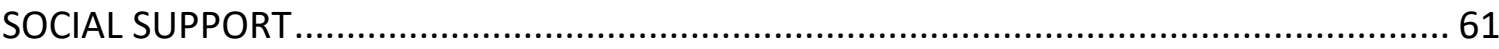

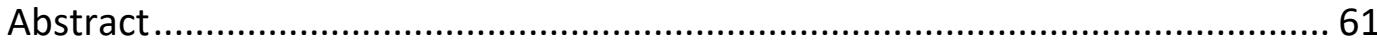

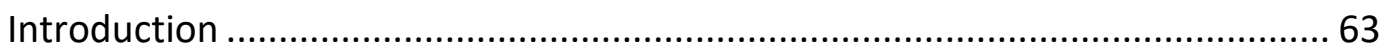

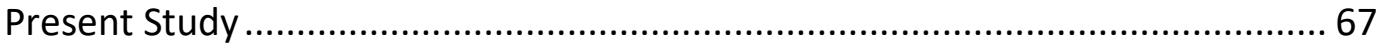

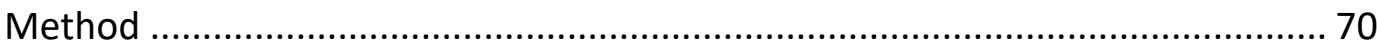

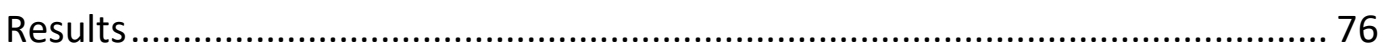

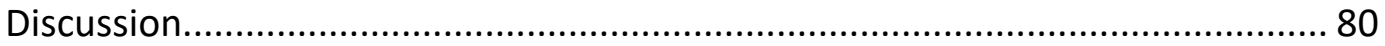

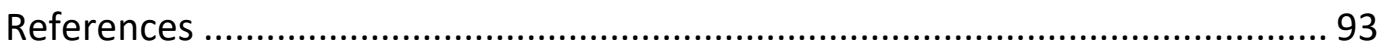


CHAPTER IV. MANUSCRIPT III. DRINKING TO COPE WITH JOB STRESS: A DAILY PROCESS INVESTIGATION OF STRESS AND COPING AMONG ACTIVE DUTY AND SEPARATED

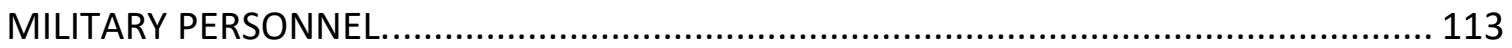

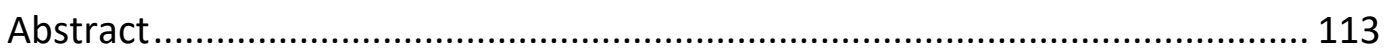

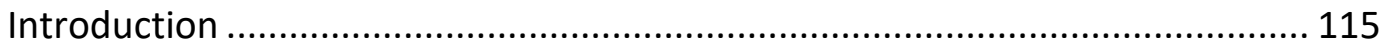

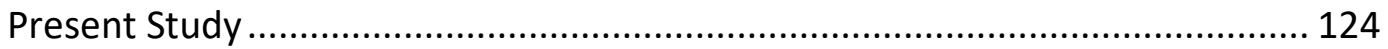

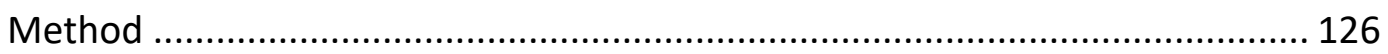

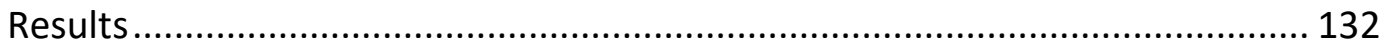

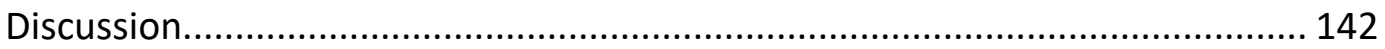

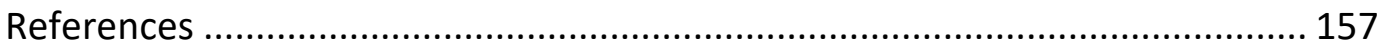

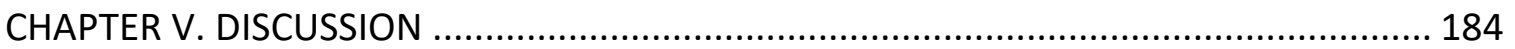

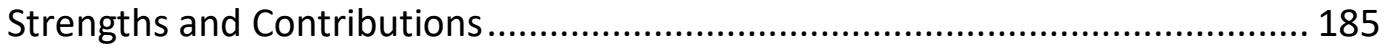

What Have We Learned About Stress and Alcohol Use?................................. 190

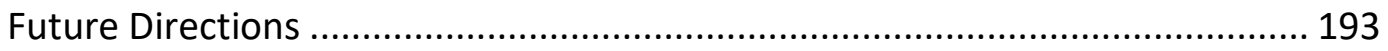

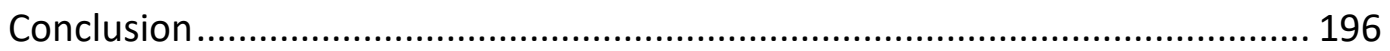

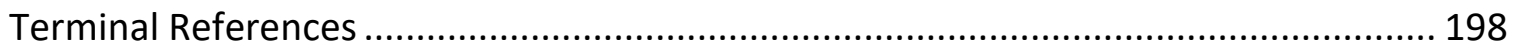

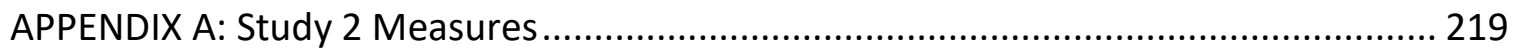

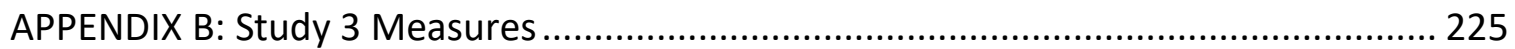


List of Tables

CHAPTER II. MANUSCRIPT I. "HAVE A DRINK, YOU'LL FEEL BETTER.” PREDICTORS OF DAILY ALCOHOL CONSUMPTION AMONG EXTRAVERTS: THE MEDIATIONAL ROLE OF COPING.

Table 2.0. Predictions of alcohol consumption from coping and extraversion in bivariate multilevel models.

CHAPTER III. MANUSCRIPT II. PTSD AND MOTIVATED ALCOHOL USE AMONG ACTIVE DUTY AND RECENTLY SEPARATED MILITARY PERSONNEL: A MODERATED MEDIATION MODEL OF SOCIAL SUPPORT

Table 3.0. Descriptive statistics for study variables.

Table 3.1. Conditional Process Analyses of the Association Between

Posttraumatic Stress Symptoms and Alcohol Involvement.

CHAPTER IV. MANUSCRIPT III. DRINKING TO COPE WITH JOB STRESS: A DAILY PROCESS INVESTIGATION OF STRESS AND COPING AMONG ACTIVE DUTY AND SEPARATED MILITARY PERSONNEL.

Table 4.0. Descriptive Statistics and Inter-Correlations Among Study Variables

Table 4.1. Multilevel Regression Results for Role Overload, Interpersonal Conflict at Work, Emotional Support Coping, and Alcohol Use 175 Table 4.2. Multilevel Regression Results for Role Overload, Interpersonal Conflict at Work, Instrumental Support Coping, and Alcohol Use 


\author{
List of Figures
}

\title{
CHAPTER I: INTRODUCTION
}

Figure 1.0. Mediation model of the association between extraversion and daily alcohol use through daily coping behaviors (problem-focused and social support coping)....

Figure 1.1. Hypothesized model of the conditional indirect effect of PTSD

symptoms on alcohol involvement through drinking to cope motives. 26

CHAPTER II. MANUSCRIPT I. "HAVE A DRINK, YOU'LL FEEL BETTER." PREDICTORS OF DAILY ALCOHOL CONSUMPTION AMONG EXTRAVERTS: THE MEDIATIONAL ROLE OF COPING.

Figure 2.0. Mediational pathway: Extraversion predicting alcohol mediated by problem-focused and social support coping.

CHAPTER III. MANUSCRIPT II. PTSD AND MOTIVATED ALCOHOL USE AMONG ACTIVE DUTY AND RECENTLY SEPARATED MILITARY PERSONNEL: A MODERATED MEDIATION MODEL OF SOCIAL SUPPORT

Figure 3.0. Hypothesized model of the conditional indirect effect of PTSD symptoms on alcohol involvement through drinking to cope motives. 109

Figure 3.1. Moderating effect of perceived support from friends (FRIENDS) on PTSD symptom-coping motives association - AUDIT model... 110

Figure 3.2. Moderating effect of perceived support from family (FAMILY) on the association between coping motives (DTC) and average number of drinks consumed..

CHAPTER IV. MANUSCRIPT III. DRINKING TO COPE WITH JOB STRESS: A DAILY PROCESS INVESTIGATION OF STRESS AND COPING AMONG ACTIVE DUTY AND SEPARATED MILITARY PERSONNEL.

Figure 4.0. Hypothesized model of role overload (RO) predicting evening alcohol use and decision to drink as moderated by drinking to cope motives (DTC) and emotional support coping (ES)

Figure 4.1.Hypothesized model of role overload (RO) predicting evening alcohol use and decision to drink as moderated by drinking to cope motives (DTC) and instrumental support coping (IS)

Figure 4.2. Hypothesized model of interpersonal conflict at work (IPC) predicting evening alcohol use and decision to drink as moderated by drinking to cope motives (DTC) and emotional support coping (ES)

Figure 4.3. Hypothesized model of interpersonal conflict at work (IPC) predicting evening alcohol use and decision to drink as moderated by drinking to cope motives (DTC) and instrumental support coping (IS) 
Figure 4.4. Marginally significant cross-level interaction between daily role overload and coping motives predicting level of evening consumption.

Figure 4.5. Marginally significant three-way cross-level interaction between daily role overload, coping motives, and average emotional support coping predicting level of evening consumption.

Figure 4.6. Three-way cross-level interaction between daily role overload, coping motives, and average instrumental support coping predicting level of evening consumption.

Figure 4.7. Marginally significant cross-level interaction between daily interpersonal conflict at work and coping motives predicting level of evening consumption..

Figure 4.8. Marginally significant three-way cross-level interaction between daily role overload, coping motives, and average emotional support coping predicting probability of drinking that evening...

\section{CHAPTER V. DISCUSSION}

Figure 5.0. Theoretical model of self-medication process... 


\section{CHAPTER I: INTRODUCTION}

Heavy and problematic alcohol consumption represents a significant public health concern. In 2012, roughly $7 \%$ of individuals 18 years or older reported heavy or problematic levels of drinking in the past month (i.e., 5 or more drinks on at least 5 occasions for men, 4 or more drinks for women; SAMHSA, 2012) with men, Caucasians, younger adults, and those with higher socioeconomic status being more likely to report heavy and binge drinking than their respective counterparts (Paul, Grubaugh, Frueh, Ellis, \& Egede, 2011). Although research has documented some benefits of moderate levels of consumption for cardiovascular health and well-being (see Kaplan et al., 2012; NIAAA, 2000), major epidemiological studies suggest there are no such benefits for heavy alcohol use. Frequent heavy drinking has been shown to contribute to poor mental and physical health functioning (NIAAA, 2000), strain within social relationships (Levitt \& Cooper, 2010; Testa \& Derrick, 2014), higher morbidity and mortality rates (Carrao, Bagnardi, Zambon, \& La Vecchia, 2004; Patra et al., 2010; Rehm, Gmel, Sempos, \& Trevisan, 2002), and an increased likelihood of engaging in other risky behaviors (Cherpitel, 1993; Cooper, 2002). The potential consequences of drinking to excess extend beyond individual health and well-being and may impact multiple ecological levels, affecting peer, family, and occupational functioning, and generating a significant economic burden (Bouchery, Harwood, Sacks, Simon, \& Brewer, 2011).

One particularly pernicious form of drinking commonly discussed in the literature pertains to the consumption of alcohol to manage or regulate stress. 
According to Conger's (1956) tension-reduction hypothesis (TRH), individuals commonly consume alcohol in order to achieve a state of reduced stress, anxiety, or tension. Indeed much work suggests alcohol may suppress or offset the psychological and physiological experience of stress (Levenson, Sher, Grossman, Newman, \& Newlin, 1980; Sher, Bartholow, Peuser, Erickson, \& Wood, 2007). Furthermore, this drive is reinforced through repeated use of alcohol to dampen the effects of stress and accompanying negative affect, thereby increasing the likelihood of drinking in similar situations. This process of drinking as a means of drive reduction contributes to the development of more abusive and problematic forms of drinking over time and simultaneously diminishes one's capacity to cope adaptively with stressors in future situations (Maisto, Carrey, \& Bradizza, 1999).

Although the claims of TRH are intuitive in nature, its efficacy in practice has been suspect to criticism. Without properly accounting for personal, historical, situational, and cognitive influences within the immediate context, the link between stress and alcohol consumption remains relatively weak (Cox \& Klinger, 1988; Sadava \& Pak, 1993). Stark differences may exist in the extent to which individuals drink in response to traumatic events and more chronic forms of stress relative to lower intensity forms of stress which may fluctuate on an ongoing basis (McCreary \& Sadava, 1998). Researchers have thus called for a more thorough accounting of the type of stressor being experienced, as well as the underlying mechanisms and conditions under which stressful experiences influence alcohol involvement (Frone, 1999; Sayette, 2000). 
It is clear much work is needed to reduce the burden of problematic alcohol use on individuals and society, and psychologists are in a unique position to contribute to this goal. In addition to traditional treatment modalities, including maintenance of more severe forms alcohol dependence (e.g., relapse prevention, cognitive behavioral therapies, medication management), primary and secondary prevention efforts that are theory driven and evidence based remain critical to provide necessary resources and education for those who have not yet begun to exert a pattern of problematic drinking and those who may be at risk for the development of future drinking problems. One area of fruitful research has identified intrapersonal factors such as motivations as key determinants of drinking behavior (Cox \& Klinger, 1988; Cooper, Russell, Skinner, \& Windle, 1992b). Such beliefs and motives which underlie and drive one's consumption in various contexts represent some of the strongest and most proximal predictors of drinking behavior (Cooper, Frone, Russell, \& Mudar, 1995; Kuntsche et al., 2005). Efforts to better understand motivational underpinnings of alcohol use and involvement will hopefully enrich our theoretical and practical understanding of the antecedent processes which influence alcohol use. To achieve this aim, and of central focus to this dissertation, a more thorough understanding is needed of (a) the underlying reasons which motivate why and how individuals are drinking, (b) individual differences in who may be at risk for engaging in problematic forms of drinking as well as (c) the availability of resources and behaviors which may serve to inhibit or reduce the likelihood of problematic forms of drinking. In this dissertation, I explored these 
issues among two such "at risk" groups for which a heavy drinking norm exists: College students, and members of the U.S. armed forces, most of whom are veterans.

Much of what is known about the psychological underpinnings of alcohol use originates from research conducted with young adult and college student populations. Recent estimates indicate that more than $40 \%$ of college student drinkers report recent binge drinking (Wechsler \& Nelson, 2008) and about $15 \%$ of college students engage in heavy drinking (NIAAA, 2014). The prevalence of these problematic patterns of use seem to be unique to the college students experience, as rates of binging and heavy drinking exceed those of other young adults who are not in college (SAMHSA, 2006). For many college students, this period represents an important life transition where they may be experiencing increased autonomy and a lack of supervision for the first time. Although attending college or university constitutes a prototypically normative progression among emerging adults in the U.S., it can be inherently stressful as individuals seek to establish their respective identities. Pressures to succeed, get good grades, make friends and fit in, and ultimately find a job or career may lead individuals to engage in risk-taking behaviors, including binge and heavy drinking. Consequently, these patterns of consumption among college students have been associated with a myriad of adverse physical, psychological, academic, and social problems unique to their experience (e.g., non-fatal injuries, risk of overdose, low self-esteem, impaired school performance, risky sexual behaviors) (Baer, 2002; Kushner \& Sher, 1993; Wechsler et al., 1998). 
Another group for which high levels of alcohol consumption represents a significant concern are military service members. In 2012, the Institute of Medicine (IOM) released a report declaring alcohol misuse in the military represents a "public health crisis" (IOM, 2012). Rates of binge and heavy drinking among service members remains significantly higher compared to their age and gender-matched non-military counterparts (Bray et al., 1991). Approximately $43 \%$ of the U.S. armed forces engaged in binge drinking in the past month (Stahre et al., 2009), and about 20\% reported heavy drinking (Bray et al., 2010), with younger, male, and members of the National Guard and Reserve being at particularly high risk for the development of heavy drinking patterns and associated problems (Jacobson et al., 2008; Milliken, Auchterlonie, \& Hoge, 2007). Alcohol has historically been used both during and following combat to help service members cope with heavy demands and stress (Jones \& Fear, 2011), and despite continued efforts to reduce high levels of consumption, has been reinforced through cultural norms, practices, and policies which increase access to alcohol and promote its use (Ames \& Cunradi, 2005). Moreover, the unique demands service members are exposed to during deployment may lead to the development of mental health symptoms (e.g., PTSD, depression, anxiety), and self-reports indicate that alcohol is often used to help mitigate these effects (Cuciarre, Darrow, \& Weingardt, 2011; Jacobson et al., 2008). High levels of alcohol use for this purpose may ultimately achieve the opposite effect over time and worsen existing mental health issues (Heslin et al., 2012), and has been implicated with impaired job performance (Adleret al., 2011; 
Fisher, Hoffman, Austin-Lane, \& Kao, 2000), increased levels of aggression (Wright, Foran, Wood, Eckford, \& McGurk, 2012), and relationship conflict including intimate partner violence (Blow et al., 2013; Foran, Heyman, Slep, \& Snarr, 2012).

\section{Motivated Drinking Patterns}

Motivational models of drinking assert that one's underlying reasons or motivations for engaging in drinking behavior (e.g., I am going to drink tonight because I want $X$ ) represent a "final common pathway" in the decision to drink and comprise a more proximal antecedent of alcohol-related outcomes relative to other factors such as positive or negative mood, stress, personality, or past family history of alcohol abuse (Cooper, 1994; Cox \& Klinger, 1988; Kuntsche et al., 2005). Indeed researchers have repeatedly demonstrated that individual motives for drinking mediate or explain the association between stressful or emotional experiences and quantity/frequency of alcohol use and associated problems (Clerkin, Werntz, Magee, Lindgren, \& Teachman, 2014; Cooper et al., 1995; Kuntsche, Wiers, Jansse, \& Gmel, 2010). Although models exist which delineate several intra- and interpersonally oriented motives for drinking such as drinking to socialize with others, to promote and extend positive experiences, to cope with stress, or to conform to situational expectations (Cooper, 1994), these motives are "phenomenologically distinct" with respect to the etiology of alcohol use, associated outcomes, and their potential consequences (Cooper et al., 1992b; Cooper, Kuntsche, Levitt, Barber, \& Wolf, in press). Of central focus to this dissertation is the important role of drinking to cope with stressful experiences. This is of critical concern 
among vulnerable populations such as college students and service members who may be experiencing a significant and stressful transitionary period in life, have been exposed to significant and traumatic stressors as a function of their occupation, or both.

Consistent with the basic tenets of TRH (Conger, 1956), one of the most widely studied motives for drinking pertains to the complex relationship between stress and subsequent alcohol use, or drinking to cope (DTC). This is perhaps unsurprising, as DTC (e.g., to forget your problems, because it helps when you feel depressed or anxious; Cooper, 1994) represents a maladaptive pattern of consumption where alcohol is consumed as a form of emotion regulation in an effort to achieve this state of diminished awareness (Hull, 1981) or to suppress/avoid aversive emotions. Collectively, motivational models have been useful in explaining the trajectory and progression of drinking patterns consistent with drinking to cope into more problematic forms of alcohol abuse and dependence. Through continued reinforcement, such behavior transitions from a volitional decision to consume alcohol to an automatic response tendency when one is exposed to events or stimuli perceived to be stressful. Some cross-sectional accounts suggest DTC is associated with heavy episodic drinking (Cooper et al., 1995; Kuntsche et al., 2005) whereas others have failed to establish this pattern, suggesting instead that DTC is associated with comparatively lower levels of drinking and drunkenness relative to other motives for drinking (e.g., positive experience enhancement) (Kuntsche et al., 2014). To the extent that one's goal in consuming alcohol is to reduce self-awareness rather than to get drunk per se, it is likely that one 
might achieve this state with low or moderate levels of consumption. Instead, drinking to cope motives are associated with a more hazardous pattern of abuse, where irrespective of drinking level, consumption of alcohol to reduce stress and negative experiences have been reliably linked with drinking alone (Cooper et al., 1992b; Mohr et al., 2001) and alcohol-related problems (Cooper et al., 1988; 1995; Kassel, Jackson, \& Unrod, 2000; Simons, Gaher, Correia, Hansen, \& Christopher, 2005). Not surprisingly, ongoing consumption in this manner produces a steep economic and personal cost in the form of deficits in physical health, relationships, and occupational functioning.

\section{Individual Differences in Stress-Related Drinking}

\section{Stressor-Vulnerability Model}

As stated earlier, experimental and cross-sectional evidence supporting the principles inherent in TRH have varied widely (Greeley \& Oei, 1999; McCreary \& Sadava, 1998; Sadava \& Pak, 1993; Sayette, 2000). Researchers have continued to refine this theory by specifying the mediating mechanisms and conditions under which stress influences drinking (Frone, 1999). One line of inquiry has shown that some individuals may be more susceptible than others to using alcohol as a form of emotion regulation (Armeli, Carney, Tennen, Affleck, \& O’Neil, 2000; Cooper et al., 1992a; Kushner, Sher, Wood \& Wood, 1994). Rooted in earlier tension-reduction theories (e.g., Conger, 1956), Cooper and colleagues (1992a) proposed a stressor-vulnerability model, identifying three individual characteristics which contribute to or increase the likelihood that one will engage in this problematic form of drinking: (1) insufficient coping skills and 
resources, (2) male gender, and (3) positive beliefs about the outcomes or

consequences of alcohol use. Along with individual-level drinking motives (e.g., coping motives), researchers have since extended Cooper's initial proposition to include certain personality traits such as neuroticism and extraversion or sensation-seeking (Cooper, Agocha, \& Sheldon, 2000), as well as low levels of social support (Catanzaro \& Laurent, 2004).

\section{Personality and Drinking}

In addition to the individual difference factors already described, research and theory suggest other individual traits, such as personality type, may predispose one to engage in high levels of drinking. The higher-order dimensions of neuroticism $(\mathrm{N})$ and extraversion (E) and their lower-ordered facets are most commonly linked with maladaptive and adaptive coping behaviors respectively, as well as drinking behaviors (Cooper et al., 2000; Feil \& Hasking, 2008). High levels of $\mathrm{N}$ are characterized by negative emotionality, and inability to effectively regulate ones emotions (David \& Suls, 1999; O'Brien \& DeLongis, 1996). Not surprisingly, these individuals may be more apt to use alcohol as a method of dampening feelings of stress and negative mood (Cooper et al., 2000; Stewart \& Devine, 2000). Conversely, individuals high in E experience higher levels of positive affect, are sociable and outgoing, and tend to engage in more direct, problem-focused forms of coping (Amirkhan, Risinger, \& Swickert, 1995; McCrae, 1992). Despite a general tendency to perceive high $\mathrm{E}$ as a ubiquitously positive trait, evidence suggests these individuals are sensitive to reward cues in their environment which may 
motivate them to engage in risk-taking behaviors, including high levels of drinking (Cooper et al., in press).

Coping Skills and Resources. According to Cooper and colleagues (1992a), "alcohol use serves as a general coping mechanism invoked when other presumably more effective coping responses are unavailable." In general, when investigating stressdrinking relationships, researchers have emphasized two major forms of coping: Approach- or problem-focused coping and avoidance coping. Individuals who engage in problem-focused coping tend to make more direct attempts at preventing, mitigating, or changing a stressful experience (Lazarus \& Folkman, 1984). This involves planning ahead, problem-solving, and seeking out new sources of information or support from others which may help alleviate stress. It is not surprising, then, that those who utilize these types of coping strategies more frequently would be less inclined to drink when stressed, as consuming alcohol would likely hinder one's performance or pursuit of a goal, and could ultimately increase stress as a result.

Conversely, individuals who engage in more avoidant strategies, such as suppressing negative emotions may be more likely to turn to alcohol use when stressed (Cooper et al., 1992a). From this perspective, alcohol may be used with the explicit intent of reducing one's level of self-awareness (Hull, 1981), thereby dampening the negative emotional impact of one's response to a stressor. Similarly, McCreary and Sadava (1998) argue that in order to resolve the discrepancy between one's current emotional state and the state they desire to achieve, the determining factor in one's 
decision to drink is based partly on the availability of alternative resources to cope with such stressors. To the extent that one lacks the appropriate skills, or they are insufficient to meet the demands of the situation, one is more likely to engage in more avoidant forms of coping, including drinking to cope (McCreary \& Sadava, 1998).

Social support resources. Social relationships represent some of the strongest resources for combatting stress. That social support has benefits for individual health and well-being is now beyond question (Cohen \& Wills, 1985; Coyne \& DeLongis, 1986; Thoits, 1995). The presence of others in times of need affords one an important source of advice and information, strategies for approaching and resolving stressful situations, or more generally a kind ear through which one can gain emotional support and understanding in times of stress. Further, the source from which the support originates (e.g., family, friends, coworkers, unit member) may be a critical determinant in the success (or failure) of supportive efforts (Halbesleben, 2006; Helgeson, 2003). For example, Catanzaro and Laurent (2004) demonstrated that although there was no direct association between support and drinking outcomes, support was shown to buffer drinking to cope behaviors among individuals who held strong beliefs that alcohol could help mitigate stress.

In addition to aiding in the effective management of minor stressors, social support may similarly reduce drinking during times of high stress (Steptoe, Wardle, Pollard, Canaan, \& Davies, 1996). In their seminal longitudinal study of college students, Steptoe and colleagues (1996) uncovered that students perceiving high levels of support 
at baseline also reported higher levels of drinking relative to lower support individuals. They explained this effect as a function of "normal" social drinking among college students. However, when examined again during a time of high stress (i.e., just before exams), perceptions of support were associated with significant reductions in reported drinking, whereas a lack of support was associated with increased drinking behavior. Similarly, among service members, emotional support has been shown to contribute to post-deployment recovery and functioning (King et al., 1998; Pietrzak et al., 2010; Wilcox, 2010), whereas lack of such support, inability to relate to others, and feelings of isolation have been associated with greater reintegration difficulties, including higher rates of posttraumatic stress disorder (PTSD) as well as increased alcohol consumption (Demers, 2011; Sayer et al., 2010).

Yet despite the benefits various forms of social support have for reducing certain harmful health behaviors, such as alcohol use (McCreary \& Sadava, 1998; Mohr, Averna, Kenny, \& Del Boca, 2001), attempts at providing support are not ubiquitously effective and may ultimately promote negative health behaviors such as alcohol use (Cohen \& LeMay, 2007; Helgeson, 2003; Shrout et al., 2006). Among groups such as college students and military service members where high levels of alcohol use are considered normative (Aldridge-Gerry et al., 2011; Neighbors, Lee, Lewis, Fossos, \& Larimer, 2007; Russell et al., 2016) supportive exchanges may revolve less around the provision of direct resources and advice, and more around the consumption of alcohol as a means of venting frustration or simply avoiding stress and negative mood for a 
short time. However, the vast majority of past research and theory does not support this view. All things being equal, perceptions, receipt, and use of social resources to cope are associated with higher well-being and health (Cohen \& Wills, 1985; Taylor, 2010). Therefore, to the extent that college students and service members are integrated into a supportive network, social support in this dissertation is believed to (a) provide critical perceptions that one is valued and cared for, and (b) provide coping resources which may aid in stress and emotion regulation. Perceptions that one is cared for, belongs, and is generally supported may buffer or protect individuals against harmful stressors by altering one's initial appraisal of the event or subsequent reaction to it (Cohen \& Wills, 1985; Taylor, 2010), or may benefit health and well-being through increases in positive mood and affect which may build further support resources and similarly aid in one's attempts at coping (Fredrickson, 2001). By their very nature, such resources may be an important source of advice and information for students and service members trying to navigate the social world.

\section{Methodological Considerations for Measuring Alcohol Involvement}

Although studies adopting a motivational approach to examining stress-drinking associations generally anticipate a positive association between DTC motives and alcohol involvement, this evidence remains mixed. Additional inconsistencies have emerged when attempting to capture these processes at the daily level, as nonsignificant findings which seem to contradict theory and past research are not uncommon (Grant, Stewart, \& Mohr, 2009; Hussong, Galloway, \& Feagans, 2005). 
Several methodological and design concerns prevent researchers from drawing important conclusions about DTC relationships, particularly with respect to conditions under which these patterns emerge. Although a majority of information on this topic has been gleaned from single time-point self-report assessments, these methods restrict the type of inquiry which can be conducted, and are unable to address key questions inherent in tension-reduction and motivational models of drinking: That is, do individuals drink more in response to experienced stress, patterns which are consistent with drinking to cope? As described earlier, implicit in transactional models of coping (e.g., Lazarus \& Folkman, 1984) as well as motivational models of drinking is the idea that individuals are responsive and reactive to the immediate situational context (Mohr et al., 2005; Tennen et al., 2000). As such, many of the topics discussed throughout this dissertation are inherently process-oriented, and cannot be accurately portrayed using cross-sectional methodologies. Rather than reflecting on past experience and behavior, the influence of stressful experiences on subsequent behaviors may be best captured in situ, as they occur.

Significant technological, statistical, and methodological developments over the past three decades have drastically altered the types of designs and approaches which can be implemented in the field. Use of ecological momentary assessments (EMAs; Stone \& Shiffman, 1994) and other daily process methods which allow for the repeated measurement of study constructs across or within days has increased rapidly as result. These methods allow for the simultaneous modeling of phenomena at multiple levels of 
analysis and have been frequently used to explore between- and within-person relationships in drinking among college students, but have only recently been applied to work with service members (see Lehavot et al., 2014; Simpson et al., 2014). By asking about phenomena in close proximity to their occurrence, these methods drastically reduce retrospection among participants, thereby increasing the reliability and accuracy of accounts (Bolger, Davis, \& Raffaeli, 2003). Additionally, daily process methods are ideally suited to capture the temporal sequencing of events such as the association between daily experiences of stress and subsequent alcohol consumption. Such temporal ordering is much more difficult to achieve using a single assessment. Importantly, when determining which level of analysis is best to address a given research question, it is perhaps most important to note that processes which occur at the between-person level do not reflect or may even disguise relationships and patterns which occur within-person or within days (Stone et al., 1998; Tennen et al., 2000). While both levels can be used to capture important information about how stressful experience relates to drinking, these levels of analysis address substantively different research questions whose effects may differ in both direction and size, thus leading to entirely different interpretations of anticipated results (Kenny, Kashy, \& Bolger, 1998). Cross-sectional and daily tests of the Stressor Vulnerability Model provide a strong illustration of these differences. In Cooper and colleagues' (1992a) seminal introduction of this model among a random sample of adults, dispositional forms of avoidant emotion-focused coping were shown to interact with both gender and alcohol use 
expectancies to predict alcohol involvement. That is, avoidant coping was significantly associated with drinking problems among men and those who held positive beliefs (i.e., expectancies) about the effects of alcohol use. Similar results have been observed by others using cross-sectional methods to examine stress-related drinking (e.g., Catanzaro \& Laurent, 2004). However, authors such as Armeli and colleagues (2000; 2007) have not found this same pattern at the daily level. Authors explained this apparent discrepancy by suggesting that when participants are asked to reflect on past coping and drinking experiences, they recall the most salient and perhaps traumatic experiences and adjust their responses accordingly (Armeli et al., 2007). To the extent that highly impactful past experiences do not match the intensity or severity of day-to-day stressors or hassles, it should not be surprising that dispositional and daily accounts of coping behavior do not align. Collectively, these studies strengthen earlier claims for the need to differentiate between- and within-person processes, as these methods describe related but distinct processes.

This discussion is not meant to suggest a lack of utility in between-person measures, rather a need to adopt more appropriate assessments when one is interested in modeling rapidly fluctuating processes such as mood, stress, and drinking behavior. Within the context of this dissertation, more stable experiences, such as major life stressors (e.g., going away to college, experiencing a traumatic event) may be more impactful on more global forms of behavior, well-being, and functioning, thus may be best captured at the person-level. In contrast, day-to-day experiences and stressors 
may be more apt to impact immediate behaviors and functioning in the moment, and may be better captured at the day level using daily process methods. As such, one of the aims of the present dissertation is to disentangle the complex associations which can be obtained by examining these experiences at multiple levels of analysis (e.g., person level, day level).

\section{Present Investigation}

Current evidence suggests individual (e.g., personality, coping resources and support, drinking motives) and contextual factors may interact to predict different forms and patterns of drinking which may be more or less problematic. These factors, particularly drinking motives (e.g., drinking to cope), represent important points for alcohol abuse prevention and intervention. However, further research is needed to explore what role these motives play in influencing everyday behavior among at risk

groups such as college students and service members. Daily process methods, wherein individuals report on their moods and behaviors at least once per day across several days are ideally suited to achieve this aim.

While much of what is known about drinking motives is based on evidence gleaned from studies with adolescents (Cooper, 1994), young adults and college students (Kuntsche et al., 2005; Mohr et al., 2005), and community-dwelling adults (Crutzen \& Kuntsche, 2013), until recently, theoretically derived models of drinking to cope have not been systematically investigated among military populations. Indeed, while evidence suggests that service members consume alcohol at rates higher than the 
general public, comparatively little is known about the psychological precipitants of such use. Self-report evidence has demonstrated that service members are cognizant of and acknowledge drinking to cope with deployment and life stress, yet with few exceptions (e.g., Lehavot et al., 2014; McDevitt-Murphy et al., 2015a; Simpson et al., 2014) it remains unclear to what extent drinking motivations, particularly coping motives influence alcohol involvement among service members. Moreover, efforts which have been undertaken have relied almost exclusively on clinical samples of treatment seeking service members.

The following three studies sought to address critical questions about alcohol involvement by exploring person- (e.g., personality, supports, mental health symptoms) and day-level (e.g., stress, coping choice) predictors of alcohol use to identify (1) which individuals may be more/less vulnerable for engaging in problematic forms of drinking, (2) how drinking to cope motivations may both explain and alter the relationship between these factors and drinking behavior. Moreover, by examining perceptions of support and support coping processes, the present studies attempted to (3) elucidate potential protective factors for more problematic forms of drinking (e.g., high quantity and frequency of use, drinking to cope, binge drinking). As heavy alcohol use and subsequent problems are prevalent across age groups (e.g., young adults and college students) and occupations (e.g., military service members; Bray et al., 2010; SAMHSA, 2006), this information will allow researchers and practitioners to better answer the question of why individuals typically drink, who may be more apt to do so, and under 
what conditions these effects hold true to develop more specific and tailored treatment modalities which incorporate and address underlying reasons for one's use.

\section{Chapter II Overview: "Have a drink, you'll feel better." Predictors of daily alcohol consumption among extraverts: The mediational role of coping.}

Study 1 is a published manuscript in the peer-reviewed journal, Anxiety, Stress, \& Coping, and examined two potential vulnerability factors for drinking, individual personality and coping behaviors. Using a daily process design, Study 1 tested the applicability of Bolger and Zuckerman's (1995) differential coping choice model to explain daily drinking behavior among college students. This model posits that, based on personality type, individuals are more or less likely to engage in certain types of coping behaviors, which lead to specific outcomes. Based on past research and theory (Bolger \& Eckenrode, 1991; DeLongis \& Holtzman, 2005), we examined the association between the personality dimension of extraversion (E) and daily drinking patterns as being mediated by two commonly reported coping strategies: (1) problem-focused coping and (2) social support. While an abundance of evidence exists with respect to the stress-buffering effects of social support (see Cohen \& Wills, 1985), socialization represents one of the primary motives for drinking among college students (Carey \& Correia, 1997; Mohr et al., 2005). As such, it was thought that support-seeking within the context of a college drinking environment may provide an opportunity for more avoidant behaviors, including heavy alcohol consumption (Aldridge-Gerry et al., 2011). 
Consistent with Bolger and Zuckerman's (1995) framework and stressorvulnerability models (see Cooper et al., 1992b), this study presented a model (see Figure 1.0) whereby the association between the personality dimension of extraversion and daily drinking patterns was mediated by individual coping choice. Specifically, it was hypothesized that the use of more direct, problem-focused strategies would be negatively associated with daily drinking, whereas social support seeking would be positively associated with daily drinking.

Chapter III Overview: PTSD and motivated alcohol use among active duty and recently separated military personnel: A moderated mediation model of social support.

Using baseline survey data from the Study for Employment Retention of Veterans (SERVe), Study 2 explored similar motivational processes to those of study 1 within a sample of military service members employed in a civilian role. The types and intensity of stressors service members experience as a function of their occupation differ markedly from those experienced by other populations, such as college student drinkers in terms of duration, type, and intensity (Nillini et al., 2014; Russell et al., 2014). Further, although drinking to cope has been identified as a prominent motivator for drinking among many service members (Cucciare et al., 2011; Jacobson et al., 2008), until recently, few have explored its direct role in influencing or determining alcohol involvement.

Study 2 represents a secondary data analysis that examined the relationship between a prominent stressor experienced by service members, namely symptoms of 
posttraumatic stress disorder (PTSS) and person-level drinking outcomes among employed military personnel. We proposed that these associations could be best explained as a function of individual level motivations for drinking, specifically drinking to cope, claims consistent with the tension-reduction model of drinking (Conger, 1956) as well as motivational models of drinking (Cooper et al., 1992b; Cox \& Klinger, 1988). Therefore, models specified that the association between PTSS experienced in the past month, and alcohol involvement (quantity and frequency of past month drinking, binge drinking) were mediated by drinking to cope motives.

An additional aim of this study was to explore potential protective factors which could ameliorate these problematic associations. A wealth of research suggests that perceptions of support can buffer the deleterious effect of stress and reduce the likelihood of one engaging in certain negative health behaviors, including alcohol use (Cohen \& LeMay, 2007; Steptoe et al., 1996). To achieve this, study 2 utilized conditional process analysis, similar to moderated mediation, to determine whether different forms of support (e.g., family and friends) moderate or buffer the link between PTSS, drinking to cope motives, and drinking outcomes (see Figure 1.1). I hypothesized that in general, social support from friends and family would have a strong influence on three underlying processes: (a) the reasons why one is drinking (e.g., to forget about their problems), (b) the strength of association between those reasons (motives) and drinking behavior, and (c) the relationship between PTSS and alcohol involvement. 
Data for this study were collected as part of the Study for Employment Retention of Veterans (SERVe), a randomized controlled trial designed to improve supports for separated service members and reservists in the workplace. Baseline data collection began in April 2014 and concluded in November 2015.

Chapter IV Overview: Drinking to cope with job stress: A daily process investigation of stress and coping among active duty and separated military personnel.

As is also described in study 2, recent evidence within the military psychology literature has emphasized the role of traumatic stress in relation to alcohol use, and have consistently shown positive associations between them at the person level (e.g., Bray et al., 2010; Jacobson et al., 2008; Kehle et al., 2012) and more recently at the daily level (e.g., Gaher et al., 2014; Lehavot et al., 2014; Simpson et al., 2014). However, much less is known about how service members react to other forms of stress they may face in their roles as civilians. As Americans spend more than half of their waking hours working (Bureau of Labor Statistics, 2014), stressful events experienced at work are likely to have a large impact on individual health and well-being (Ganster \& Rosen, 2013; Sonnentag \& Frese, 2013). To our knowledge, no studies have explored the role of daily experiences of occupational stress as they relate to daily drinking behavior among service members, and only four other studies (see Lehavot et al., 2014; McDevittMurphy et al., 2015a; Simpson et al., 2014; Whiteman \& Barry, 2011) have applied Cooper's (1994) framework to explore the role of drinking motives among military service members. 
Using a sample of married or cohabiting service members from Study 2, Study 3 utilized secondary data from a 32-day sub-study of military couples (SERVe Daily Family Study) to examine how two forms of occupational stress (i.e., role overload and interpersonal conflict at work) influenced daily drinking behaviors among an employed military sample. Moreover, this study sought to determine whether these associations varied as a function of person-level drinking to cope motive. Based on past research, it was hypothesized that coping motives would moderate stress-alcohol associations, such that daily stress-drinking associations would be stronger among those endorsing higher levels of coping motives.

Along with studies 1 and 2, and consistent with past research and theory, study 3 similarly explored the protective role of interpersonal relationships in this process. Specifically, I achieved this by modeling the extent to which seeking emotional support or advice from others to solve a problem (i.e., instrumental support) to cope across the study period buffered stress-drinking associations. Three-way, cross-level interactions were tested between occupational stress, support coping, and drinking to cope motives predicting daily alcohol use.

\section{Summary and Significance}

Collectively, these studies provide evidence about the interplay of stress, individual traits, and psychosocial factors on health behaviors at multiple levels of analysis (i.e., person-level and day-level). Study 1 examined the role of coping strategies in explaining observed associations between personality type and daily alcohol use 
among college students. Studies 2 and 3 in particular extend the existing knowledgebase with respect to alcohol use among military service members by exploring stressrelated drinking in response to more chronic (e.g., PTSS) and acute forms of stress (e.g., daily job stress) at the between- and within-person levels respectively. Study 2 sought to test a sophisticated model through which the commonly observed association between PTSS and alcohol involvement (IOM, 2014; Thomas et al., 2010) could be explained as a function of service member endorsement of drinking to cope motives. Furthermore, I examined psychological processes of support buffering (Cohen \& Wills, 1985) to determine whether these associations varied as a function of one's perceived availability of support. Although the study was cross-sectional in nature, such work offers a potential explanation for why rates of comorbidity between PTSD and alcohol misuse are so high among service members, and for whom these associations hold. Similar to study 2 , study 3 built on past research describing the role of drinking to cope motives in influencing daily job stress-drinking associations by applying these theoretically and empirically validated frameworks to a new population, military service members. Much interest has remained in reducing problematic drinking this population despite the relative dearth of evidence with respect to motivated drinking patterns. This study is among the first to explore the role of person-level motives as they relate to the daily drinking patterns, and the first to explore such issues as a product of occupational stressors using a community sample of service members employed in a civilian role. 
In addition to the theoretical significance of these studies, further elucidation of the role of support coping and drinking motivations in daily behavior of service members has important implications for prevention and intervention (Cooper et al., in press; Jacobson et al., 2008; Kuntsche et al., 2005). Drinking motives are a final pathway through which mental health, stress, and other socioemotional factors influence behavior (Cox \& Klinger, 1988), thus represent important targets for treatment efforts. 


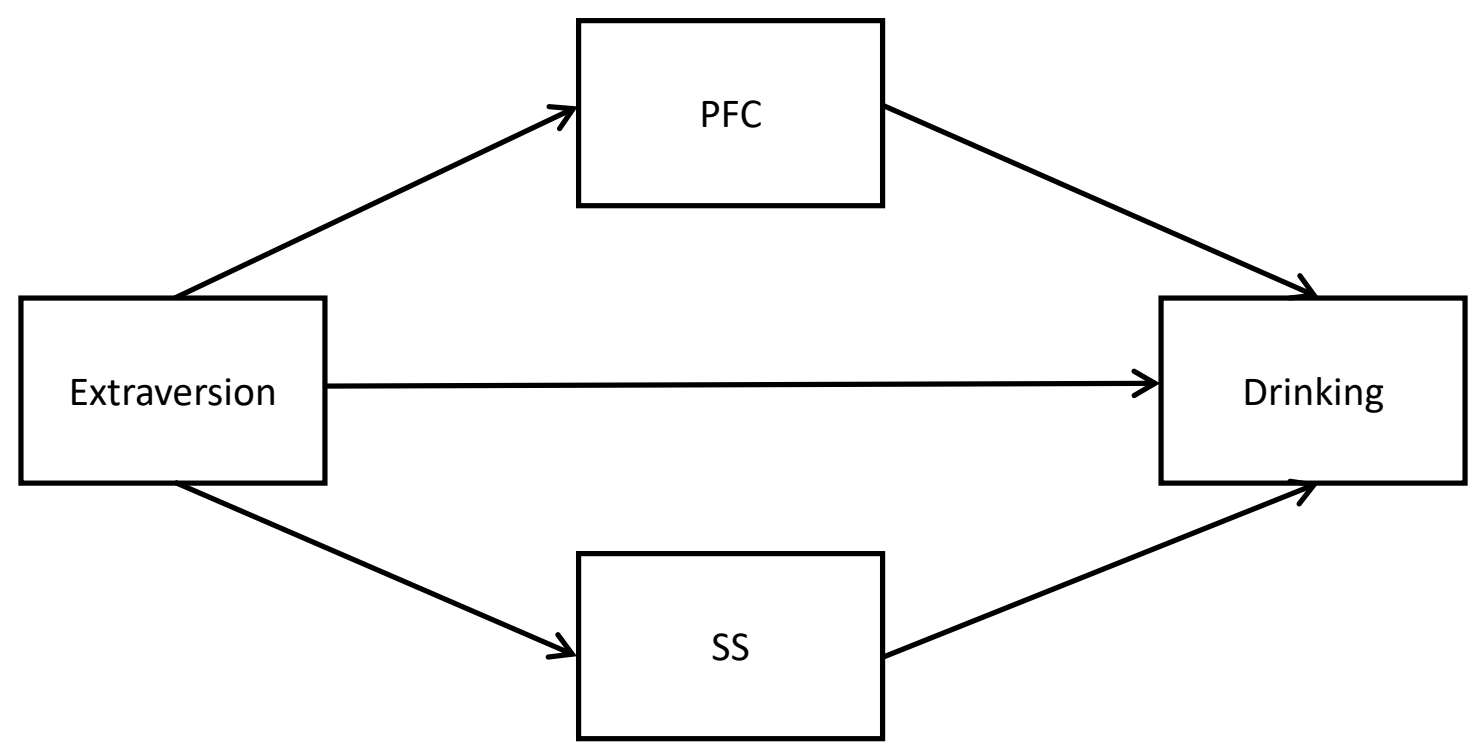

Figure 1.0. Mediation model of the association between extraversion and daily alcohol use through daily coping behaviors (problem-focused and social support coping)

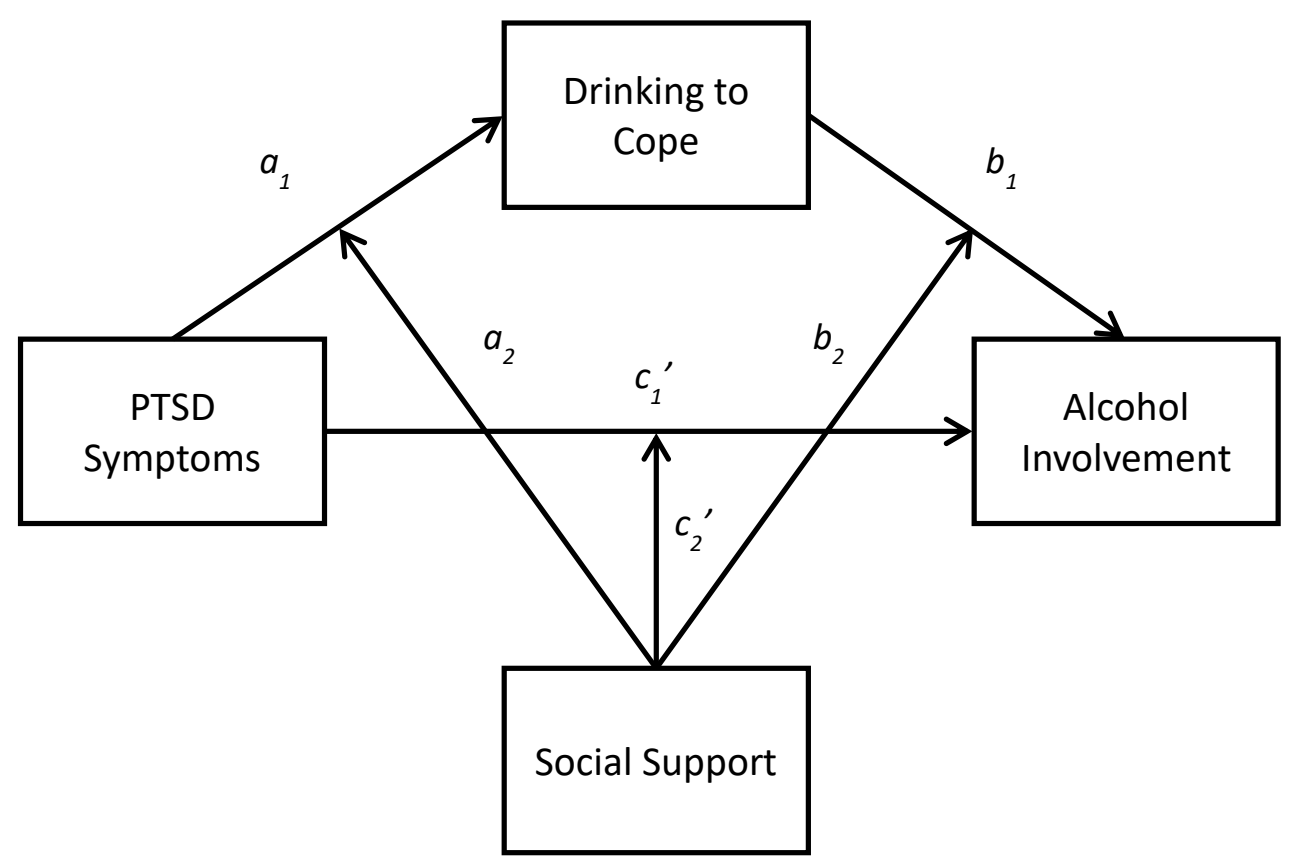

Figure 1.1. Hypothesized model of the conditional indirect effect of PTSD on alcohol involvement through drinking to cope motives. 
VULNERABILITY AND PROTECTIVE FACTORS OF STRESS-DRINKING

CHAPTER II. MANUSCRIPT I. "HAVE A DRINK, YOU'LL FEEL BETTER." PREDICTORS OF DAILY ALCOHOL CONSUMPTION AMONG EXTRAVERTS: THE MEDIATIONAL ROLE OF COPING.

\begin{abstract}
An abundance of information exists pertaining to individual differences in college drinking behaviors with much attention being provided to the role of personality. However, plausible explanations for what prompts engagement in or avoidance of these behaviors have remained largely ambiguous or underexplored, particularly with respect to extraversion. Research has since explored how coping behaviors contribute to these associations. The present study built on this research by evaluating differences in daily alcohol consumption as a function of coping choice. The mediational effects of two specific strategies frequently observed in high extraversion individuals (i.e., problemfocused coping and social support) were examined. Using a daily diary approach, 365 undergraduates reported their most stressful experience, how they coped with it, and the number of drinks consumed for five consecutive days. Resulting multilevel-models were consistent with hypotheses indicating the relationship between extraversion and alcohol consumption was partially mediated by problem-focused and support-seeking strategies. The use of problem-focused coping by high extraversion individuals was associated with lower levels of daily alcohol consumption, suggesting this strategy may play a protective role in influencing drinking behaviors. Conversely, the positive effect observed for social support approached significance $(p=.054)$ and was indicative of a potential risk-factor for daily alcohol consumption.
\end{abstract}


Keywords: college students, alcohol consumption, coping, extraversion, daily diary 


\section{Introduction}

College students have been shown to consume more alcohol relative to similar age groups who did not attend college (Substance Abuse and Mental Health Services Administration, 2006) and may be at higher risk for several physical, psychological, academic, and social problems (Baer, 2002; Kushner \& Sher, 1993; Stewart \& Devine, 2000). Despite the abundance of existing information on individual differences in college drinking behaviors, plausible explanations for what prompts engagement in or avoidance of these risky behaviors have remained largely ambiguous or underexplored, particularly with respect to the dimension of extraversion (E) who may be susceptible to heavy alcohol use and other risky behaviors given the social and impulsive aspects of their personality (Jackson \& Matthews, 1988). Researchers have since explored individual motivations behind drinking as a potential link between personality traits and alcohol consumption (Cooper, 1994; Cooper, Frone, Russell, \& Mudar, 1995; Cox \& Klinger, 1988), particularly the motivation to cope with stress (see Britton, 2004; Cooper, Russell, Skinner, Frone, \& Mudar, 1992; Feil \& Hasking, 2008 among others). Most studies linking personality and alcohol consumption, however have measured trait-based or dispositional coping behaviors (Connor-Smith \& Flachsbart, 2007; David \& Suls, 1999; Suls \& Martin, 2005) and few (if any) have addressed on how these individuals cope with daily stressors and how the selection or choice of coping strategies may serve to inhibit or increase levels of daily alcohol consumption. 


\section{Coping and Personality}

Coping has been defined simply as any attempt at regulating one's emotional state, regardless of the effectiveness of the attempts (Folkman et al., 1986). The transactional model of stress and coping views coping as a conscious process that varies with the demands of a given situation (Lazarus \& Folkman, 1987) and places less importance on trait-based dispositional forms of coping, rather the process of how individuals attend, appraise, and react to a given stressor (Suls \& David, 1996). Events perceived as controllable denote more proactive attempts towards stress reduction (problem-focused coping) while events seen as uncontrollable yield attempts to modify how one responds to a stressor (emotion-focused coping) (Carver \& Connor-Smith, 2010; David \& Suls, 1999; Lee-Baggley et al., 2005), highlighting the importance of situational and contextual determinants of behavior. However, consistency of the fit between appraisal and coping choice has been shown to vary significantly within persons, and may be more pronounced with problem-focused strategies (Park, Armeli, \& Tennen, 2004; Park, Folkman, \& Bostrom, 2001).

Of the five major personality dimensions (see McCrae \& Costa, 1987) one of the most intriguing but also misunderstood dimensions in the coping literature is that of extraversion (E). High $\mathrm{E}$ is often associated with positive emotionality, assertiveness, vibrancy, sociability, and optimism (McCrae, 1992; McCrae \& Costa, 1987; Robinson, Solberg, Vargas, \& Tamir, 2003). These high E individuals tend to appraise situations as challenging rather than threatening (Amirkhan, Risinger, \& Swickert, 1995; Carver \& 
Connor-Smith, 2010; Lee-Baggley et al., 2005), to engage in more proactive or problemfocused attempts at coping (Amirkhan et al., 1995; De Longis \& Holtzman, 2005) as well as adaptive forms of emotion-focused coping (Lee-Baggley et al., 2005; McCrae \& Costa, 1986), and tend to utilize fewer maladaptive and avoidant strategies (Amirkhan et al., 1995; Carver \& Connor-Smith, 2010; Lee-Baggley et al., 2005 . Moreover, high E individuals experience fewer stressors overall and do not react as strongly when they do (Connor-Smith \& Flachsbart, 2007; Gunthert, Cohen, \& Armeli, 1999; Suls \& Martin, 2005).

Given their high levels of sociability (McCrae \& Costa, 1987), it is no surprise that high E individuals approach others for support in times of duress (Amirkhan et al., 1995; Bolger \& Eckenrode, 1991; Swickert, 2009; Swickert, Rosentreter, Hittner, \& Mushrush, 2002). Social support has received significant attention in the coping literature regarding its role in the coping process; specifically as to which coping domain it falls under (problem or emotion-focused). In a recent review of personality and coping literature, Carver and Connor-Smith (2010) argued this distinction may be situationallydetermined. Social support seeking may be considered problem-focused if the goal is to obtain advice regarding how to approach a problem/stressor, or emotion-focused if the goal is to obtain emotional support following a problem/stressor (Carver \& ConnorSmith, 2010). Research has shown when the type of support provided does not match or is inappropriate given the stressor one is exposed to, social support may become 
ineffective or maladaptive, leading to certain risky behaviors including alcohol consumption (Cutrona, Shaffer, Wesner, \& Gardner, 2007; Thoits, 1995).

\section{Extraversion, Coping, and Alcohol Consumption}

According to the Tension-Reduction Hypothesis (Conger, 1956), alcohol consumption allows for temporary relief from daily stressors, thereby reinforcing certain faulty coping strategies (Mohr et al., 2001). Furthermore, consuming alcohol in an attempt to regulate negative affect (i.e., to cope) has been linked with solitary drinking, heavy episodic drinking, avoidance drinking, and alcohol related problems. Among college students, research has not only observed more alcohol related problems among those who drink to cope than those who drink for enhancement or social motives (Kassel, Jackson, Shannon, \& Unrod, 2000), but also higher quantities of drinks being consumed per occasion as well (Britton, 2004). Continued use of alcohol to cope with one's negative affect may deteriorate adaptive coping skills thereby predisposing them to drink more when stressed which can lead to future alcohol dependence (Cooper, 1994; Cooper et al., 1995; Field \& Quigley, 2009; Gonzalez et al., 2009; Stewart \& Devine, 2000).

The use of proactive coping strategies has been shown to be efficient methods by which individuals can reduce deleterious effects of stress on both health and wellbeing (Lazarus, 2006). Specifically as it relates to alcohol use, problem-focused coping has been linked with both lower levels of consumption (Feil \& Hasking, 2008) and also reported problems (McCreary \& Sadava, 1998). These direct forms of coping are among 
the most commonly employed by high E individuals (Amirkhan et al., 1995; Carver \& Connor-Smith, 2010; Lee-Baggley et al., 2005). This supports the idea that those high in E may be less likely to engage in stress drinking and are less likely to experience problems associated with heavy alcohol use. However, researchers claim that other features (i.e., sensation seeking and impulsivity; Baer, 2002; Grau \& Ortet, 1999; Jackson \& Matthews, 1988) observed in high E may lead to increased alcohol use. These individuals are motivated by social relationships (Hussong, 2003; Kuntsche, von Fischer, \& Gmel, 2008), and tend to seek out others when stressed. Within the college drinking environments, support seeking among high E individuals may provide means by which they can avoid a potential stressor, and has been linked with greater alcohol consumption (Aldridge-Gerry et al., 2011). Moreover, research has shown those high in E tend to drink more heavily in environments where drinking is condoned and social interaction is expected (Cooper et al., 1992; Fischer, Smith, Anderson, \& Flory, 2003; Hussong, 2003).

\section{Measuring Coping in Situ}

Criticisms of prior conceptualizations of coping have been that most studies have focused heavily on the experience of major life stressors (Bolger, DeLongis, Kessler, \& Schilling, 1989) or have looked primarily at dispositional measures of coping (trait-based coping) which do not accurately reflect the nuances and impacts of daily stressors, and relationships that should exist are often overshadowed (Connor-Smith \& Flachsbart, 2007). Moreover, these models are most often derived from cross-sectional reports 
which forces one to recall prior stressful experiences and how one has coped; the accuracy of which diminishes rapidly (Armeli, Todd, \& Mohr, 2005). Cross-sectional accounts may also fail to detect minor fluctuations which can occur between and also during onsets of stressful events (DeLongis \& Holtzman, 2005).

Recent research has inspired a shift towards the adoption of a model coping which is a state-based, contextually dependent process (Lazarus, 2006; Lazarus \& Folkman, 1986) and have attempted to disentangle these situational and contextual issues by measuring coping in situ using a daily diary methodology (e.g., DeLongis \& Holtzman, 2005; Lee-Baggley et al., 2005). Daily or minor stressors are not only more readily occurring than major life events, but also require more immediate attention (Bolger et al., 1989). Daily reports of stress and coping allow for an in-depth examination into underlying processes associated with coping (Mohr et al., 2003), place less strain on participants having to recall previous coping attempts, thus systematically reducing retrospective bias and subsequently yield more accurate results regarding the relationship between coping and personality (Connor-Smith \& Flachsbart, 2007; David \& Suls, 1999; Suls \& Martin, 2005). These daily assessments have helped clarify prior inconsistencies in the personality and coping literature such as $E$ being unrelated to problem-focused coping (O’Brien \& DeLongis, 1996) or adaptive emotion-focused coping (including social support; David \& Suls, 1999; O’Brien \& DeLongis, 1996). 


\section{Present Study}

Consistent with transactional models of coping behavior, Bolger and Zuckerman (1995) reasoned that mere exposure to varying types of stressors does not allow for adequate prediction of outcomes; rather individual reactions to stressful experiences provide the most useful information (see also Bolger \& Schilling, 1991). Evidence from their study supports a mediational model (i.e., coping choice) in which individuals (based on personality dimension) are predisposed to cope in certain ways, and those strategies lead to specific outcomes (Bolger \& Zuckerman, 1995).

Using a daily diary approach, the present study tested the applicability of Bolger and Zuckerman's (1995) differential coping choice model in a sample of undergraduate college students. Focusing on the dimension of extraversion, we expected high $\mathrm{E}$ individuals (relative to low E) to be more likely to employ problem-focused strategies and seek the support of their social network when faced with stressful events. Additionally, measuring alcohol consumption as our primary outcome, we expected to find evidence that coping mediated the relationship between $\mathrm{E}$ and alcohol use. More specifically, we believe individuals high in $E$ (relative to low $E$ ) who report using problem-focused coping will also report lower frequency of alcohol use. Conversely those high in $\mathrm{E}$ (relative to those lower in E) who use social support should report higher frequency of alcohol use. 


\section{Method}

\section{Participants}

Participants were college students recruited from a large western university. Three hundred and sixty-six participants completed all target measures (described below). There were more female than male participants (68.5\% vs. $31.5 \%)$ and ages ranged from 17 to 25 years $(M=20.14, S D=2.10)$. This multiethnic sample was composed of Caucasians (37.6\%), Asian Americans (30.6\%), Hispanics/Latinos (20.7\%), African Americans (9.1\%), and individuals who were either biracial or some other ethnic group (2\%). The sample also represented a cross-section of majors at the university, with larger percentages of Business (24.0\%) and Psychology (15.9\%) majors, respectively. Moreover, $51 \%$ of the participants were 1 st year students.

\section{Measures}

Daily diary pages assessed three primary variables: Stress, coping, and alcohol consumption. Personality and demographic variables were completed at one administration point.

Perceived stress/controllability. Participants were asked to first describe the most stressful or bothersome event that had occurred during each day using an openended format. These events were classified according to type of stressful event (e.g., academic, peer relationship, parent relationship). Next the participants rated the perceived stressfulness of the event using a 5-point rating scale $(1=$ very slightly to $5=$

extremely). Additionally, participants were asked their level of perceived controllability 
over this stressful experience ("How much do you feel you can control the outcome of this event?"), on a 5 -point scale ( 1 = no control to 5 = absolute control).

Coping. Daily coping was assessed with 28 items reflecting 14 specific coping strategies using a 4-point scale $(1=$ not at all to $4=a$ lot $)$. These items were taken from the Brief COPE (Carver, 1997), the Children's Coping Strategies Checklist and the How I Coped Under Pressure Scale (Ayers \& Sandler, 2000) and the Responses to Stress Questionnaire (Connor-Smith, Compas, Wadsworth, Thomsen, \& Saltzman, 2000). Two daily coping variables were used based on a recent multilevel factor analysis (see Roesch et al., 2010 for a full exposition of the use of this technique and derivation of the factors): (1) Social Support (mean $\alpha=.77$; composed of problem-focused and emotionfocused support items; e.g., talked to my friends about how I was feeling); and (2) Problem-Focused Coping (mean $\alpha=.80$; composed of problem solving and cognitive decision making items; e.g., thought about what I need to know to solve the problem).

Alcohol consumption. The total number of standard drinks consumed per day was calculated from daily reports of the number of drinks consumed. The scale was modeled after Armeli and colleagues (2006) daily measure of alcohol consumption. Prior to initiating daily reports, participants were familiarized with the concept of a standard drink and instructed about the volumes of different beverages and their equivalents to a standard drink (i.e., 12-oz beer, 4-oz glass of wine, or 1-oz glass of spirits). For convenience, instructions were also provided to participants on the daily questionnaire. 
Personality questionnaire. To assess the dimension of $E$, the 10 -item E scale from the International Personality Item Pool (IPIP; Goldberg, 1999) was used. Instructions asked participants to rate how accurately each of the items described them using a 5 -point scale ranging from $1=$ very inaccurate to $5=$ very accurate $(\alpha=.88)$ using statements like "I make friends easily" and "I have little to say".

\section{Procedure}

Participants were recruited via flyers, course/club presentations, and university seminars. Once an individual agreed to participate they received instructions (via email) on how to complete the web-based daily diary page over the course of five days. Webbased diary studies have been shown to yield similar results to both lab (Birnbaum, 2004) and paper-based studies (Green et al., 2006). Potential participants signed an electronic informed consent prior to participating in the study. Participants then completed the IPIP and the demographic questionnaire and were given instructions on how to complete the web-based daily diary page over the next 5 consecutive days. Participants were given a username and password (that they could change) to access the secured website in order to complete the diary page. These procedures are consistent with recent web-based daily diary studies (Nezlek, 2005; Park et al., 2004). Compliance with the diary page at the end of the day was high, with the modal response time of reporting being 9:43 PM and over $85 \%$ of observations reported after 7 PM. Participants were paid $\$ 25$ at the completion of the study. 
Preliminary Data Analyses. After preliminary data screening and cleaning, multilevel modeling was used to analyze the data according to the particular hypotheses specified (see Nezlek, 2001; Raudenbush \& Bryk, 2002). The primary analyses were conducted on two-level models. The measures of alcohol consumption, and coping from the daily diary page are considered lower-level (level-1) variables, whereas personality factors are considered higher-level (level-2) variables. Level-1 predictor variables were group-mean centered. Thus, aggregate versions of the target level-1 predictors were also added to the intercept equation at level-2 to account for between-individual variance. Time of day, day of week, generational status, gender, and perceived stress (from the daily diary page) were evaluated for use as covariates in the target analyses. All analyses were conducted using HLM 6.06.

\section{Results}

Descriptive Statistics A total of 1,760 daily diary observations were completed for the 365 participants, with close to $30 \%$ of observations falling on a weekend (defined as Friday through Sunday). Participants in this study had a mean extraversion score of $3.36(S D=.74)$. Exposure to daily stressors yielded an average perceived stressfulness of $3.57(S D=1.08)$ while the mean perceived controllability of those events was $2.91(S D=$ 1.18). Of the coping strategies of interest (i.e., problem-focused and seeking social support), participants primarily used problem-focused coping $(M=2.63, S D=0.84)$ with lesser use of social support strategies $(M=1.88, S D=0.82)$. One-hundred sixty five participants (approximately $45 \%$ ) reported consuming alcohol at least once during the 
five reporting days. Students consumed alcohol 322 of the 1,760 total reporting days (approximately $18 \%$ of observed days) with an average number of drinks consumed per occasion of $3.73(S D=3.26)$.

Multilevel Regression Models. Bivariate analyses were conducted to test for significant covariates with the target outcome variable (alcohol). Level-1 predictors included the day of assessment, perceived control over stressor, perceived stressfulness, and a dummy-coded variable comparing weekday vs. weekend (weekday referent group). Level-2 included dummy-coded versions of gender (male as referent group), ethnic comparisons of minority groups (Asian, Hispanic/Latino, African American) to Caucasians, and also age. All continuous covariate variables were grand mean centered and treated as fixed effects. A multilevel Poisson model was used given that alcohol is a count variable with a positive skew $(M=0.67, S D=1.99)$. A log-link function with an over-dispersion parameter was used due to the high standard deviation. Individual level results indicate that male gender and older age (event rate $[\mathrm{ER}]=0.70,95 \% \mathrm{Cl}[0.53,0.94], p=.017 ;$ and $\mathrm{ER}=1.11,95 \% \mathrm{Cl}[1.04,1.18], p=.002$ respectively) are predictive of greater alcohol consumption among undergraduates whereas minority group membership was associated with lower levels of alcohol consumption relative to Caucasians (African Americans, $\mathrm{ER}=0.46,95 \% \mathrm{Cl}[0.31,0.70], p$ $<.001 ;$ Hispanic/Latinos, $\mathrm{ER}=0.58,95 \% \mathrm{Cl}[0.44,0.78], p=.001 ;$ Asian Americans, $\mathrm{ER}=$ $0.55,95 \% \mathrm{Cl}[0.37,0.80], p=.002)$. Only weekend (vs. weekday) was significantly associated with daily alcohol consumption (ER $=3.42,95 \% \mathrm{Cl}[2.84,4.12], p<.001)$. 
These statistically significant covariates were included in all subsequent predictive models of alcohol consumption.

In order to reveal the mediational influence of coping on the extraversion/alcohol consumption relationship, a direct link was tested between extraversion and alcohol consumption controlling for the significant covariates. A significant direct effect for extraversion was found, such that those high in extraversion drank significantly more relative to those lower in extraversion, controlling for the significant covariates. This effect can be interpreted by evaluating the exponentiated log-link function of the regression equation.

$$
\begin{aligned}
& \ln (\text { Alcohol' })=B_{0}+B_{1} E \\
& e^{\ln \left(\text { Alcohol' }^{\prime}\right)}=e^{(-.570+.304 E)}
\end{aligned}
$$

This equation allows for the prediction of the quantity of alcohol an individual consumes based on their score from the extraversion scale. For example, a participant with an extraversion score of 4.1 (one standard deviation above the mean), is predicted to consume approximately two drinks per occasion (1.97), while someone with an extraversion score of 2.62 (one standard deviation below the mean) is predicted to drink approximately 1.25 drinks per occasion.

This same set of covariates was used to test the second portion of our mediational model, the association between our coping variables and extraversion (antecedent to mediator paths). While none of the individual-level predictors were associated with the use of problem-focused coping or social support, daily-level 
predictors such as higher perceived controllability $(B=0.23, p<.001)$ and higher perceived stress $(B=0.12, p<.001)$ were associated with greater use of daily problemfocused coping, whereas only higher perceived stress was associated with the use of social support $(B=0.13, p<.001)$. The use of problem-focused coping $(B=-0.05, p<$ $.001)$ and social support seeking $(B=-0.09, p<.001)$ decreased over the five assessment days. Once E was added to the model, the antecedent to mediator paths from both E to problem-focused coping $(B=0.12, p=.003)$ and social support $(B=0.14, p=.001)$, respectively, were statistically significant.

Finally, to test whether the mediator to outcome relationships were statistically significant, a final model was tested. Both individual level coping variables were specified as predictors of alcohol consumption; the corresponding aggregate (meanlevel) versions of coping were simultaneously entered into the model predicting alcohol consumption to remove any between-person variability. Thus, the individual level coping variables representing daily use (rather than aggregate use) of coping were included in these analyses. Daily coping variables were entered into the Level-1 equation (group-mean centered) while the corresponding aggregate variables were entered at Level-2 (grand-mean centered). E was also added as a level 2 predictor of alcohol consumption.

As shown in Table 2.0, significant individual level predictors of alcohol consumption included gender, all ethnic comparisons, age, and extraversion $(E R=1.40$, 95\% Cl $[1.16,1.69], p=.001)$, while mean-level problem-focused and social support 
coping were not significant ( $p=.542$ and .100 respectively). There was a statistically significant and negative association between daily level use of problem-focused coping predicting alcohol consumption for this sample $(\mathrm{ER}=.79,95 \% \mathrm{Cl}[.678, .921], p=.003)$. Conversely, the association of social support seeking with alcohol consumption was positive and approached significance $(\mathrm{ER}=1.16,95 \% \mathrm{Cl}[.998,1.35], p=.054$; see Figure 2.0 for a full description of effects).

\section{Discussion}

Results from this daily diary study add further to the literature suggesting a complex link between the personality dimension of extraversion, coping strategies, and alcohol consumption. While positive personality qualities of being optimistic, sociable and cheerful (Amirkhan et al., 1995; Lucas \& Baird, 2004; McCrae, 1992; O’Brien \& DeLongis, 1996) can typically predict positive outcomes, they may mask certain behaviors which leave those high in extraversion susceptible to risky behaviors. In the past, researchers have explained two primary traits of high extraversion which contribute to differing levels of alcohol consumption as well as the development of substance use: impulsivity and sociability (Acton, 2003). Moreover, impulsivity has been shown to be a vulnerability factor for alcohol use in both experimental and crosssectional studies (Acton, 2003; Cooper, Agocha, \& Sheldon, 2000; Grau \& Ortet, 1999; Mezquita et al., 2010). Overall, results from this study coincide with previous research that individuals who reported higher levels of extraversion consumed more alcohol per 
day relative to those reporting lower levels of extraversion (see also Acton, 2003; Baer, 2002; Fischer et al., 2003; Martsh \& Miller, 1997).

Positive appraisals of stress (Amirkhan et al., 1995; Carver \& Connor-Smith, 2010) and ample resources to defend against it suggest those high in extraversion are more likely to employ problem-focused coping strategies. As predicted, scores on the extraversion scale were positively associated with the use of these problem-focused strategies to cope with daily stressors. These strategies had a direct and negative association with alcohol consumption suggesting the use of problem-focused coping strategies at the daily level predict lower levels of same-day alcohol consumption. While evidence for full-mediation of the extraversion to alcohol relationship by coping was not supported in this study, results do suggest that problem-focused coping partially mediates this relationship and suggest that daily or ongoing use of this strategy may serve to buffer levels of alcohol consumption for high extraversion individuals.

Along with displaying more problem-focused strategies, researchers have consistently shown the importance of interpersonal relationships and support seeking among high extraversion individuals (Amirkhan et al., 1995; Connor-Smith \& Flachsbart, 2007; Lee-Baggley et al., 2005; Swickert et al., 2002). Results from this study coincide with previous research, indicating that scores on the extraversion scale were positively associated with the use of social support seeking strategies to cope with daily stressors. Although the use of social support coping and social engagement have most often been associated with positive outcomes, including better health outcomes (Ozer \& Benet- 
Martínez, 2006), this study found the association between social support and increased alcohol consumption was near significance. Although this effect failed to meet traditional statistical significance standards, this finding may be of practical importance as research has shown that peers provide a strong influence on drinking patterns, particularly among college students (Baer, 2002).

\section{Limitations}

Given the focused nature of the research questions asked in this study, several limitations should be addressed. While the primary focus of this paper was to present new evidence regarding how extraversion specifically relates to both coping and alcohol outcomes, future research may profit from evaluating all five dimensions of personality as research has shown that differences in how these dimensions present themselves and interact across situations may provide important substantive insight into the relationship between personality, coping, and alcohol use (DeLongis \& Holtzman, 2005; Gunthert et al., 1999; Mezquita et al., 2010).

Another limitation arose from the single measure of alcohol consumption. Although the total number of standard drinks consumed per occasion was modeled after a validated measure of alcohol consumption (see Armeli et al., 2006), it may have benefitted from additional measures related to overall alcohol use. Despite this limitation, the current alcohol use measure was selected to specifically assess levels of daily alcohol consumption. 
While the state-based effects of perceived stressfulness and controllability of stress were statistically controlled for in this study, the impact of their effects was not interpreted. These factors have been shown to play a role in the utilization of different coping strategies (David \& Suls, 1999; Folkman et al., 1986, Park et al., 2004).

Moreover, the type of stressor encountered may play a crucial role in the selection of coping strategy and its resulting effectiveness (Cutrona \& Russell, 1990, Cutrona et al., 2007; Troits, 1995). Future research should be mindful of the impact of these factors and may benefit by testing for their role as potential moderators of the coping-alcohol relationship.

Although not measured directly, given the college environment, the effect of social support on levels of alcohol consumption may be at least partially indicative of a motivation to socialize with others rather than a desire to alleviate stress, per se, although investigation of this theory is beyond the scope of the present paper. Future research might also benefit from the inclusion of these state-based items assessing contextual as well as motivational factors with the goal of teasing apart individual motivations for drinking from actuarial coping habits. This focus on context and situation has been implemented by researchers like Mohr and colleagues (2008) who discussed how drinking at home or alone is linked with increased use of alcohol to cope with stress and one's own negative affect (see also Christiansen, Vik, \& Jarchow, 2002; Cooper, 1994), whereas drinking elsewhere (e.g., at a party or bar) is most typically 
associated with enhancement and social motives for drinking (Cooper et al., 1992;

Cooper, 1994; Hussong, 2003; MacLean \& Lecci, 2000).

Another potential source of bias in this study was its use of self-report measures. However, according to Chan (2009), these reports are necessary to evaluate selfreferential perceptions (e.g., how one has coped) although could be supplemented by additional measures. Finally, the generalizability of these results to different samples should also be considered. Participants in this study represent a limited age range and demographic, and thus cannot be generalized to the population at large.

\section{Conclusion}

Overall, these findings add to the growing literature measuring coping on a day to day basis. While there is an abundance of literature pertaining to college drinking patterns, there is less information available regarding how these patterns are influenced by how one copes with daily stressors and even less as to how this relates to those who score high on extraversion specifically. Personality traits are enduring features which influence individual outlooks, beliefs, and behaviors. These "social-butterflies" have been depicted in a relatively positive light in personality and coping research, and indeed, according to this study effective use of problem-focused strategies towards

diminishing stress may serve to reduce alcohol consumption among college students high in extraversion. However, those who tend to seek out the company of friends while stressed may be predisposed to engage in certain risky behaviors, including increased alcohol consumption. Results from this study may be used to inform 
interventions and programs geared toward reducing college drinking. Particularly as it relates to those higher in extraversion, interventions should emphasize and promote problem-focused coping skills such as planful problem-solving or proactive coping strategies to diminish stress-related drinking. Interventions should also seek to provide individual support and guidance for regulating more impulsive behaviors which may supersede adaptive behaviors and lead to more stress related drinking.

\section{Acknowledgments}

This research was supported by National Institute of Child Health and Human Development grant F31HD056623 to Arianna A. Aldridge and National Institute of Mental Health grant MH065515 to Scott C. Roesch. 


\section{References}

Acton, G. S. (2003). Measurement of impulsivity in a hierarchical model of personality traits: Implications for substance use. Substance Use \& Misuse, 38, 67-83.

Aldridge-Gerry, A. A., Roesch, S. C., Villodas, F., McCabe, C., Leung, Q. K., \& Da Costa, M. (2011). Daily stress and alcohol consumption: Modeling between-person and within-person ethnic variation in coping behavior. Journal of Studies on Alcohol and Drugs, $72,125-134$.

Amirkhan, J. H., Risinger, R. T., \& Swickert, R. J. (1995). Extraversion: A “hidden” personality factor in coping? Journal of Personality, 63, 189-212.

Armeli, S., Feinn, R., Tennen, H., \& Kranzler, H.R. (2006). Effects of naltrexone on alcohol consumption and affect reactivity to daily interpersonal events among heavy drinkers. Experimental and Clinical Psychopharmacology, 14, 199-208.

Armeli, S., Todd, M., \& Mohr, C. (2005). A daily process approach to individual differences in stress-related alcohol use. Journal of Personality, 73 (6), 16571686.

Ayers, T., \& Sandler, I. N. (2000). The Children's Coping Strategies Checklist and The How I Coped Under Pressure Scale. Unpublished manual. Arizona State University.

Baer, J. S. (2002). Student factors: Understanding individual variation in college drinking. Journal of Studies on Alcohol, 14, 40-53.

Birnbaum, M. (2004). Human research and data collection via the internet. Annual Review of Psychology, 55, 803-832. 
Bolger, N., DeLongis, A., Kessler, R. C., \& Schilling, E. A. (1989). Effects of daily stress on negative mood. Journal of Personality and Social Psychology, 57, 808-818.

Bolger, N., \& Eckenrode, J. (1991), Social relationships, personality, and anxiety during a major stressful event. Journal of Personality and Social Psychology, 61, 440-449.

Bolger, N., \& Schilling, E. A. (1991). Personality and the problems of everyday life: The role of neuroticism in exposure and reactivity to daily stressors. Journal of Personality, 59, 355-386.

Bolger, N., \& Zuckerman, A. (1995). A framework for studying personality in the stress process. Journal of Personality and Social Psychology, 69, 890-902.

Brennan, A. F., Walfish, S., \& AuBuchon, P. (1986). Alcohol use and abuse in college students: I. A review of individual and personality correlates. International Journal of Addiction, 21, 449-474.

Britton, P. C. (2004). The relation of coping strategies to alcohol consumption and alcohol-related consequences in a college sample. Addiction Research and Theory, 12, 103-114.

Carver, C.S. (1997). You want to measure coping but your protocol's too long: Consider the Brief COPE. International Journal of Behavioral Medicine, 4, 92-100.

Carver, C. S., \& Connor-Smith, J. (2010). Personality and Coping. Annual Review of Psychology, 61, 679-704. 
Chan, D. (2009). So why ask me? Are self-report data really that bad? In C. E. Lance \& R. J. Vandenberg (Eds.), Statistical and methodological myths and urban legends: Doctrine, verity, and fable in the organizational and social sciences (pp. 303-336). New York, NY: Routledge.

Christiansen, M., Vik, P., \& Jarchow, A. (2002). College student heavy drinking in social contexts versus alone. Addictive Behaviors, 27, 393-404.

Conger, J. J. (1956). Alcoholism: Theory, problem and challenge: II. Reinforcement theory and the dynamics of alcoholism. Quarterly Journal of Studies on Alcohol, $17,296-305$.

Connor-Smith, J.K., Compas, B.E., Wadsworth, M.E., Thomsen, A.H., \& Saltzman, H. (2000). Responses to stress in adolescence: Measurement of coping and involuntary stress responses. Journal of Consulting and Clinical Psychology, 68, 976-992.

Connor-Smith, J. K., \& Flachsbart, C. (2007). Relations between personality and coping: A meta-analysis. Journal of Personality and Social Psychology, 6, 1080-1107.

Cooper, M. L. (1994). Motivations for alcohol use among adolescents: Development and validation of a four-factor model. Psychological Assessment, 6, 117-128.

Cooper, M. L., Agocha, V. B., \& Sheldon, M. S. (2000). A motivation perspective on risky behaviors: The role of personality and affect regulatory processes. Journal of Personality, 68, 1059-1088. 
Cooper, M. L., Frone, M. R., Russell, M., \& Mudar, P. (1995). Drinking to regulate positive and negative emotions: A motivational model of alcohol use. Journal of Personality and Social Psychology, 69, 990-1005.

Cooper, M. L., Russell, M., Skinner, J. B., Frone, M. R., \& Mudar, P. (1992). Stress and alcohol use: Moderating effects of gender, coping, and alcohol expectancies. Journal of Abnormal Psychology, 101, 139-152.

Cox, M., \& Klinger, E. (1988). A motivational model of alcohol use. Journal of Abnormal Psychology, 97, 168-180.

Cutrona, C. E., \& Russell, D. (1990). Type of social support and specific stress: Toward a theory of optimal matching. In I. G. Sarason, B. R. Pierce (Eds.), Social support: An interactional view (pp. 219-366). New York: Wiley.

Cutrona, C. E., Shaffer, P. A., Wesner, K. A., \& Gardner, K. A. (2007). Optimally matching support and perceived spousal sensitivity. Journal of Family Psychology, 21, 754758.

David, J. P., \& Suls, J. (1999). Coping efforts in daily life: Role of Big Five traits and problems appraisals. Journal of Personality, 67, 265-294.

DeLongis, A., \& Holtzman, S. (2005). Coping in context: The role of stress, social support, and personality in coping. Journal of Personality, 73, 2-24.

Feil, J., \& Hasking, P. (2008). The relationship between personality, coping strategies and alcohol use. Addiction Research and Theory, 16, 526-537. 
Field, M., \& Quigley, M. (2009). Mild stress increases attentional bias in social drinkers who drink to cope: A replication and extension. Experimental and Clinical Psychopharmacology, 17, 312-319.

Fischer, S., Smith, G. T., Anderson, K. G., \& Flory, K. (2003). Expectancy influences the operation of personality on behavior. Psychology of Addictive Behaviors, 17, 108114.

Folkman, S., Lazarus, R. S., Dunkel-Schetter, C., DeLongis, A., \& Gruen, R. J. (1986). Dynamics of a stressful encounter: Cognitive appraisal, coping, and encounter outcomes. Journal of Personality and Social Psychology, 50, 992-1003.

Goldberg, L.R. (1999). A broad-bandwidth, public-domain, personality inventory measuring the lower-level facets of several five-factor models. In I. Mervielde, I. Deary, F. De Fruyt, \& F. Ostendorf (Eds.), Personality Psychology in Europe: Vol. 7 (pp. 7-28). Tilburg, The Netherlands: Tilburg University Press.

Gonzalez, V. M., Bradizza, C. M., \& Collins, R. L. (2009). Drinking to cope as a statistical mediator in the relationship between suicidal ideation and alcohol outcomes among underage college drinkers. Psychology of Addictive Behaviors, 23, 443451.

Grau, E., \& Ortet, G. (1999). Personality traits and alcohol consumption in a sample of non-alcoholic women. Personality and Individual Differences, 27, 1057-1066.

Gray, J. A. (1981). A critique of Eysenck's theory of personality. In H. J. Eysenck (Ed.), A Model for Personality (pp. 246-276). NY: Springer. 
Green, A. S., Rafaeli, E., Bolger, N., Shrout, P. E., \& Reis, H. T. (2006). Paper or plastic? Data equivalence in paper and electronic diaries. Psychological Methods, 11, 87105.

Greeley, J., \& Oei, T. (1999). Alcohol and tension reduction. In K. E. Leonard \& H. T. Blane (Eds.), Psychological theories of drinking and alcoholism (2nd ed., pp. 14-53). New York: Guilford Press.

Gunthert, K. C., Cohen, L., \& Armeli, S. (1999). The role of neuroticism in daily stress and coping. Journal of Personality and Social Psychology, 77, 1087-1100.

Hotard, S. R., McFatter, R. M., McWhirter, R. M., \& Stegall, M. E. (1989). Interactive effects of extraversion, neuroticism, and social relationships on subjective wellbeing. Journal of Personality and Social Psychology, 57, 321-331.

Hussong, A. M. (2003). Social influences in motivated drinking among college students. Psychology of Addictive Behaviors, 17, 2, 142-150.

Jackson, C. P., \& Matthews, G. (1988). The prediction of habitual alcohol use from alcohol related expectancies and personality. Alcohol and Alcoholism, 23, 305314.

Kassel, J. D., Jackson, S. I., Shannon, I., \& Unrod, M. (2000). Generalized expectancies for negative mood regulation and problem drinking among college students. Journal of Studies on Alcohol, 61 (2), 332-357. 
Kuntsche, E., von Fischer, M., \& Gmel, G. (2008). Personality factors and alcohol use: A mediator analysis of drinking motives. Personality and Individual Differences, 45, 796-800.

Kushner, M. G., \& Sher, K. J. (1993). Comorbidity of alcohol and anxiety disorders among college students: Effects of gender and family history of alcoholism. Addictive Behaviors, 18, 543-552.

Larsen, R. J., \& Ketelaar, T. (1991). Personality and susceptibility to positive and negative emotional states. Journal of Personality and Social Psychology, 61, 132-140.

Lazarus, R. S. (2006). Emotions and interpersonal relationships: Toward a personcentered conceptualization of emotions and coping. Journal of Personality, 74, 946.

Lazarus, R.S., \& Folkman, S. (1987). Transactional theory and research on emotions and coping. European Journal of Personality, 1, 141-169.

Lee-Baggley, D., Preece, M., DeLongis, A. (2005). Coping with interpersonal stress: Role of Big Five traits. Journal of Personality, 73, 1141-1180.

Littlefield, A. K., Sher, K. J., \& Wood, P. K. (2009). Is "maturing out" of problematic alcohol involvement related to personality change? Journal of Abnormal Psychology, 118, 360-374.

Lucas, R. E., \& Baird, B. M. (2004). Extraversion and emotional reactivity. Journal of Personality and Social Psychology, 86, 473-485. 
MacLean, M. G., \& Lecci, L. (2000). A comparison of models of drinking motives in a university sample. Psychology of Addictive Behaviors, 14,83-87.

Martsh, C. T. \& Miller, W. R. (1997). Extraversion predicts heavy drinking in college students. Personality and Individual Differences, 23, 153-155.

McCrae, R. R. (Ed.). (1992). The five-factor model: Issues and applications [Special issue]. Journal of Personality, 60(2), 175-215

McCrae, R. R., \& Costa, P. T., Jr. (1986). Personality, coping, and coping effectiveness in an adult sample. Journal of Personality, 54, 385-405.

McCrae, R. R., \& Costa, P. T., Jr. (1987). Validation of the five-factor model of personality across instruments and observers. Journal of Personality and Social Psychology, $52,81-90$

McCreary, D. R., \& Sadava, S. W. (1998). Stress, drinking, and the adverse consequences of drinking in two samples of young adults. Psychology of Addictive Behaviors, $12,247-261$.

Mezquita, L., Stewart, S. H., \& Ruipérez, M. Á. (2010). Big-Five personality domains predict internal drinking motives in young adults. Personality and Individual Differences, 49, 240-245.

Mohr, C. D., Armeli, S., McCauley Ohannessian, C., Tennen, H., Carney, A., Affleck, G., \& Del Boca, F. K. (2003). Daily interpersonal experiences and distress: Are women more vulnerable? Journal of Social and Clinical Psychology, 22, 393-423. 
Mohr, C. D., Armeli, S., Tennen, H., Carney, M. A., Affleck, G., \& Hromi, A. (2001). Daily interpersonal experience, context, and alcohol consumption: Crying in your beer and toasting good times. Journal of Personality and Social Psychology, 80, 489500.

Mohr, C. D., Brannan, D., Mohr, J., Armeli, S., \& Tennen, H. (2008). Evidence for positive mood buffering among college student drinkers. Personality and Social Psychology Bulletin, 34, 1249-1259.

Nezlek, J. B. (2001). Multilevel random coefficient analyses of event- and intervalcontingent data in social and personality psychology research. Personality and Social Psychology Bulletin, 27, 771-785.

Nezlek, J. B. (2005).Distinguishing affective and non-affective reactions to daily events. Journal of Personality, 73, 1539-1568.

O’Brien, T. B., \&DeLongis, A. (1996). The interactional context of problem-, emotion-, and relationship-focused coping: The role of the Big Five personality factors. Journal of Personality, 64, 775-813.

Ozer, D. J., \& Benet-Martínez (2006).Personality and the prediction of consequential outcomes. Annual Review of Psychology, 57, 552-567.

Park, C.L., Armeli, S., \& Tennen, H. (2004). Appraisal-coping goodness of fit: A daily internet study. Personality and Social Psychology Bulletin, 30, 558-569. 
Park, C. L., Folkman, S., \& Bostrom, A. (2001). Appraisals of controllability and coping in caregivers and HIV+ men: Testing the goodness-of-fit hypothesis. Journal of Consulting and Clinical Psychology, 69, 481-488.

Raudenbush, S.W., \& Bryk, A. S. (2002). Hierarchical Linear Models: Applications and Data Analysis Methods ( $\left.2^{\text {nd }} \mathrm{Ed}\right)$. Thousand Oaks, CA: Sage Publications.

Robinson, M. D., Solberg, E. C., Vargas, P., \& Tamir, M. (2003). Trait as default: Extraversion, subjective well-being, and the distinction between neutral and positive events. Journal of Personality and Social Psychology, 85, 517-527.

Roesch, S.C., Aldridge, A.A., Stocking, S.N., Villodas, F., Leung, Q., Bartley, C.E., \& Black, L.J. (2010). Multilevel factor analysis of daily diary coping data: Modeling trait and state variation. Multivariate Behavioral Research, 45, 767-789.

Stewart, S. H., \& Chambers, L. (2000). Relationships between drinking motives and drinking restraint. Addictive Behaviors, 25, 269-274.

Stewart, S. H., \& Devine, H.(2000). Relations between personality and drinking motives in young adults. Personality and Individual Differences, 29, 495-511.

Substance Abuse and Mental Health Services Administration. (2006). Results from the 2005 National Survey on Drug Use and Health: National Findings, DHHS Publication No. SMA 06-4194. Rockville, MD: Office of Applied Studies.

Suls, J., \& David, J. P. (1996). Coping and personality: Third time's the charm? Journal of Personality, 64, 993-1005. 
Suls, J., \& Martin, R. (2005). The daily life of the garden-variety neurotic: Reactivity, stressor exposure, mood spillover, and maladaptive coping. Journal of Personality, 73, 1-25.

Swickert, R. J., Rosentreter, C. J., Hittner, J. B., \& Mushrush, J. E. (2002). Extraversion, social support processes, and stress. Personality and Individual Differences, 32, 877-891.

Swickert, R. (2009). Personality and social support processes. In P. J. Corr \& G. Matthews (Eds.), The Cambridge handbook of personality psychology, (pp. 524-540). New York, NY: Cambridge University Press.

Thoits, P. (1995). Stress, coping, and social support processes: Where are we? What next? Journal of Health and Social Behavior, extra issue, 53-79. 
Table 2.0

Predictions of alcohol consumption from coping and extraversion in bivariate multilevel models.

\begin{tabular}{lrrr}
\hline & & \multicolumn{2}{l}{ Event rate } \\
Predictor & $B(S E)$ & $p$ & \multicolumn{1}{l}{$[95 \% \mathrm{Cl}]$} \\
\hline Weekend vs. Weekday & $1.27(0.10)$ & $<.001$ & $3.54[2.93,4.28]$ \\
Gender & $-0.40(0.15)$ & .009 & $0.67[0.50,0.90]$ \\
Ethnic comparisons (vs. Caucasian) & & & \\
$\quad$ Af. American & $-0.72(0.20)$ & .001 & $0.48[0.33,0.72]$ \\
$\quad$ Hispanic/Latino & $-0.48(0.14)$ & .001 & $0.62[0.47,0.81]$ \\
$\quad$ Asian American & $-0.58(0.19)$ & .003 & $0.56[0.39,0.82]$ \\
Age & $0.14(0.03)$ & $<.001$ & $1.15[1.07,1.23]$ \\
Extraversion & $0.34(0.10)$ & .001 & $1.40[1.16,1.69]$ \\
Daily Problem-Focused Coping & $-0.24(0.08)$ & .003 & $0.79[0.68,0.92]$ \\
Daily Social Support & $0.15(0.08)$ & .054 & $1.16[1.00,1.35]$ \\
Person-level problem-focused coping & $-0.08(0.13)$ & .542 & $0.92[0.72,1.19]$ \\
Person-level social support & $0.17(0.10)$ & .100 & $1.19[0.97,1.45]$ \\
\hline
\end{tabular}

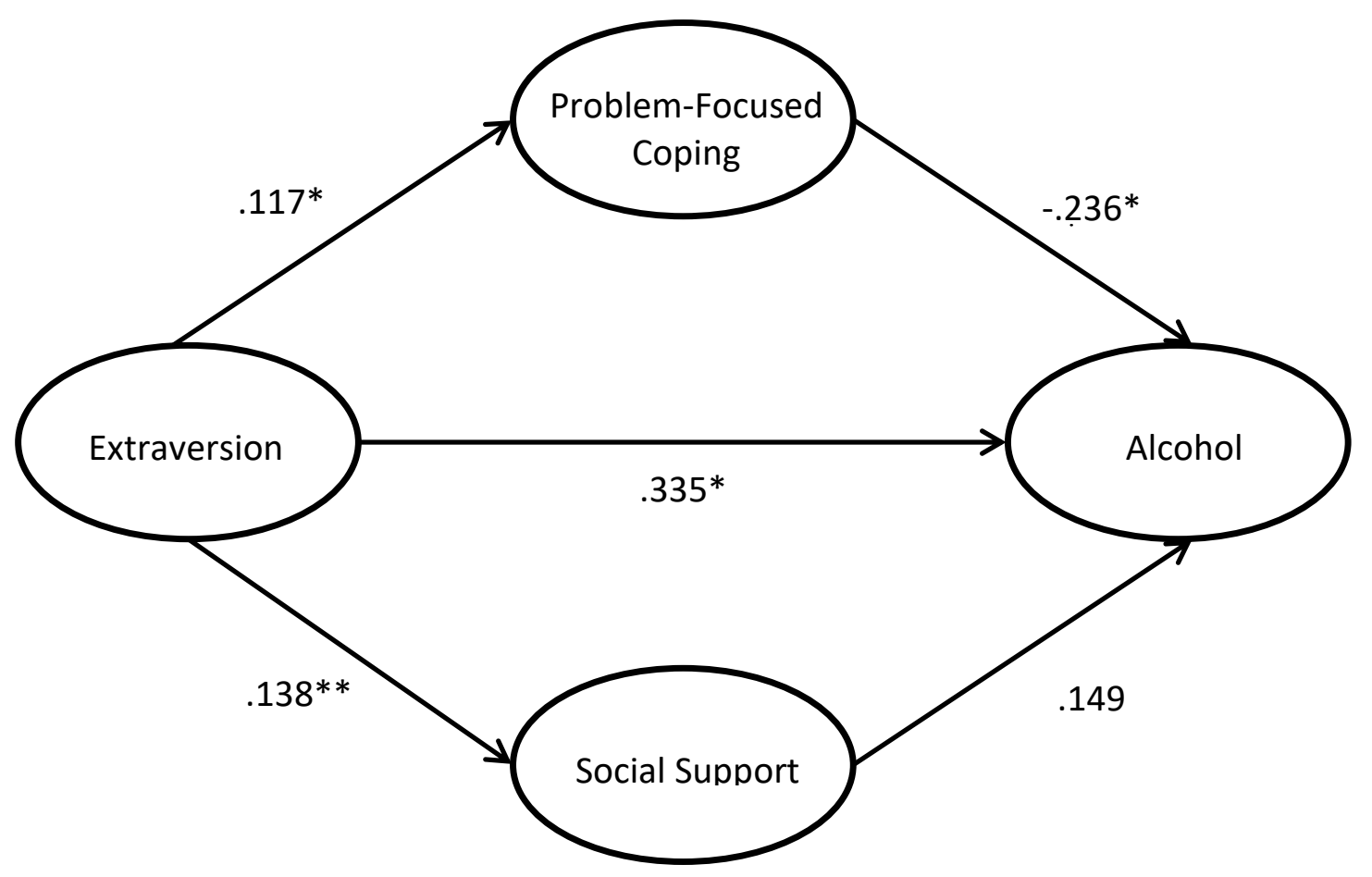

Figure 2.0. Mediational pathway: Extraversion predicting alcohol mediated by problem-focused and social support coping.

* Significant at the .01 level

** Significant at the .001 level 
VULNERABILITY AND PROTECTIVE FACTORS OF STRESS-DRINKING

CHAPTER III. MANUSCRIPT II. PTSD SYMPTOMOLOGY AND MOTIVATED ALCOHOL USE AMONG MILITARY SERVICE MEMBERS: A CONDITIONAL INDIRECT EFFECT MODEL OF SOCIAL SUPPORT

\begin{abstract}
Comorbidity between posttraumatic stress disorder (PTSD) and problematic alcohol use among military service members presents numerous challenges for health and wellbeing. With 2.7 million service members having served since 2002 , it is critical to understand both why these patterns emerge, and how they can be mitigated. One potential mechanism through which this association can be explained is the underlying motivations service members hold for drinking, particularly drinking to cope motives. The primary aim of the present study was to determine whether the association between PTSD symptoms (PTSS) and alcohol involvement (defined as quantity and frequency of use, alcohol problems, and binging) could be explained through the endorsement of drinking to cope motives. A secondary aim was to identify potential protective factors, such as social support, which may mitigate these associations. As part of a longitudinal project examining health and well-being among civilian-employed separated service members and reservists, the present study investigated these issues using a sample of 398 current drinkers. Using conditional process analysis, eight conditional indirect effect models examined whether PTSS-alcohol associations were indirect through the endorsement of coping motives, and whether this indirect effect was conditional upon perceptions of support from family and friends. Coping motives fully mediated associations between PTSS and alcohol involvement. Results of
\end{abstract}


conditional indirect effects revealed discrepant results whereby perceived support from friends weakened PTSS-coping motives associations when predicting drinking problems, whereas perceived support from family strengthened coping motives-average drinking quantity associations. Future screening and intervention efforts should attend to individual motivations for drinking as important determinants of alcohol-related outcomes among service members experiencing PTSS, and emphasize the importance of communication, trust, and effective supports among military and nonmilitary friends and family.

Keywords: military service members, PTSD, alcohol, drinking to cope motives, social support 


\section{Introduction}

It is well established that events experienced during military deployments can lead to deleterious mental and physical health outcomes (Hoge et al., 2007; Schnurr \& Spiro, 1999). Posttraumatic stress disorder (PTSD) is a mental health disorder characterized by reliving the traumatic experience, hypervigilance for suspected threats, repeated intrusive thoughts and negative thoughts or cognitions, and withdrawal from people and events associated with trauma (American Psychiatric Association, 2013; King, King, Foy, Keane, \& Fairbank, 1999), and is among the most common concerns facing service members. Prevalence of PTSD among service members from Operations Enduring Freedom and Iraqi Freedom (OEF/OIF) is estimated at about 20\% (Tanielian \& Jaycox, 2008), although reported rates have ranged from $8 \%(I O M, 2014)$ to as high as 41\% (Sayer et al., 2010) in large-scale studies. PTSD is unique when compared to other mental health disorders in that it can be tied to a specific cause or event (McNally, 2003). Importantly, the types of events and stressors experienced by service members while deployed in combat settings can differ markedly from events and stressors experienced by non-military populations in terms of their duration and intensity (Russell et al., 2014), which may partially explain why PTSD occurs more commonly among service members relative to their civilian counterparts. Further, rates of PTSD differ within military populations, as the prevalence is higher among those with a higher level and severity of exposures during deployment (Hoge et al., 2004; Nillni et al., 2014), and more generally based on one's service branch (Adler et al., 2011; Gorman, Blow, Ames, 
\& Reed, 2011) and role within the military (e.g., infantry, medic, engineer; Sundin et al 2010).

In addition to mental health concerns experienced by some service members, hazardous alcohol use is a significant issue in need of attention (IOM, 2012).

Comorbidity between PTSD and alcohol misuse or problems is high (IOM, 2014; Thomas et al., 2010). Studies have shown the prevalence of more hazardous forms of drinking are highest among those who have been deployed, seen high levels of combat, or who are experiencing mental health concerns such as PTSD (Bray et al., 2013; Jacobson et al., 2008; Stahre, Brewer, Fonseca, \& Naimi, 2009). One mechanism researchers have theorized to explain this link is that alcohol is being consumed as a method of coping with or avoiding PTSD symptoms (PTSS) (Jacobson et al., 2008). Evidence is mounting in support of this perspective, as recent investigations have shown a high proportion of service members reporting use of drugs and alcohol for the explicit purpose of coping with PTSS (e.g., nightmares, flashbacks, sleep disturbances) and stress or depression (Cucciare, Darrow, \& Weingardt, 2011).

Motivational models of alcohol use (e.g., Cooper, 1994; Cox \& Klinger, 1988) claim that individual reasons or motives for drinking are among the most proximal determinants of alcohol involvement (i.e., quantity and frequency of use, alcohol problems). Such motives represent a "final pathway" through which stressful and emotional experiences influence drinking behavior (Cox \& Klinger, 1988), and lead to unique and distinct alcohol use behaviors and consequences. According to the tension- 
reduction hypothesis (Conger, 1956; Greeley \& Oei, 1999), one of the strongest motivations for consuming alcohol is to avoid or reduce aversive emotional experiences, or to achieve a state of diminished awareness. From this perspective, individuals who are motivated to drink to cope do so as a form of mood or affect regulation, and are more likely to drink alone (Cooper, Russell, Skinner, Frone, \& Mudar, 1992) and to report alcohol problems, irrespective of quantity and frequency of actual consumption (Cooper, Frone, Russell, \& Mudar, 1995; Cooper, Russell, \& George, 1988; Kuntsche et al., 2005; Simons et al., 2005). That is, regardless of how much or often one drinks, if they are generally motivated to do so in order to dampen effects of PTSS, stress, or negative mood, this can lead to greater problems with drinking. Continued and consistent consumption of alcohol in this manner may achieve the opposite of the desired effect by exacerbating mental health symptoms and diminishing one's ability to effectively manage stressful situations in the future (Burnett-Zeigler et al., 2011; Maisto, Carey, \& Bradizza, 1999; McCarthy \& Petrakis, 2010).

Although much research on tension-reduction drinking has been conducted over the last half century, researchers have only recently begun to formally investigate theoretically driven patterns of motivated drinking among military populations (Lehavot et al., 2014; McDevitt-Murphy et al., 2015a; Simpson et al., 2014). Using a sample of veterans participating in a brief intervention study for hazardous drinkers, McDevittMurphy and colleagues (2015a) demonstrated that motivations for drinking could be distinguished by PTSD status. Specifically, those with PTSD reported significantly greater 
motives for drinking to cope with depression and also anxiety relative to those without PTSD. Similarly, veterans with PTSD reported greater consequences associated with their use of alcohol, despite not reporting higher levels of consumption (i.e., drinks per week, drinking days, and heavy drinking episodes).

\section{Social Support and Health}

Despite the intuitive nature of claims inherent in the tension-reduction hypothesis, a number of contextual factors have been shown to alter stress-drinking relationships. Past research and theory suggest some individuals may be more susceptible than others to drinking in response to stressful experiences, including PTSS (Cooper et al., 1992; Sadava \& Pak, 1993; Sayette, 2000). In contrast, certain protective factors such as social relationships and supports may attenuate or buffer the deleterious effects of PTSS, thereby reducing the motivation or need to drink. Among service members, such supportive factors aid in the post-deployment recovery and functioning (King et al., 1998; Pietrzak et al., 2010; Russell et al., 2016, Wilcox, 2010), whereas lack of such support, inability to relate to others, and feelings of isolation have been associated with greater reintegration difficulties and higher prevalence of PTSD and alcohol consumption following deployment (Sayer et al., 2010).

Yet attempts at providing support are not uniformly effective and the type counsel one receives may actually serve to promote negative health behaviors, including alcohol use (Cohen \& LeMay, 2007; Helgeson, 2003). The source of support, particularly family and friends, is important to consider as they may be differentially effective in 
promoting health and well-being (Cohen \& Wills, 1985; Dakof \& Taylor, 1990; Uchino, Cacioppo, Kiecolt-Glaser, 1996). In their 2010 study of combat deployed veterans, Pietrzak and colleagues showed that post-deployment support from one's family, friends, and community along with support and cohesion within one's military unit contribute to veteran resilience and are negatively associated with PTSS and depressive symptoms. Another study of U.S. Army Special Operations personnel found that support from friends (but not family) buffered the association between combat experiences while deployment and PTSS (Russell et al., 2016). Further, such supports have been shown to facilitate and promote posttraumatic growth (Maguen, Vogt, King, King, \& Litz, 2006). Thus, research should attend to the perceived availability of such resources among service members, as support and understanding from close others (e.g., family, close friends) may provide an important outlet to offset or even prevent the development of mental health concerns.

\section{Present Study}

Individual motivations or reasons for drinking along with social supports represent two critical points of intervention for reducing problematic drinking among service members (Jacobson et al., 2008; Norman, Schmied, \& Larson, 2014). These factors act as potential determinants of why service members are drinking (e.g., to cope with PTSS) and can help identify individuals who may be at risk for engaging in more problematic forms of drinking (e.g., those who perceive low levels of support). Using a non-clinical sample of past-month drinkers recruited as part of a larger randomized- 
controlled trial designed to improve workplace support for separated service members and reservists, the present study examined a moderated mediation model whereby the relationship between PTSS and alcohol involvement (e.g., quantity and frequency of use, alcohol problems) is explained through drinking to cope motivations. We anticipated the direct associations between PTSS, coping motives, and drinking outcomes would be differentially affected by one's level of perceived support from friends and family. That is, does the explanatory role of drinking to cope motives on PTSS-alcohol involvement associations change as a function of one's perceived level of support (e.g., does support from close others buffer the desire to drink to cope)? Figure 3.0 (see Appendix I) depicts the hypothesized moderated mediation models. Based on earlier models of mediation and moderation (e.g., Baron \& Kenny, 1986) and the recommendations of Hayes (2013) for conducting conditional process analysis (i.e., moderated mediation), the present study examined six interrelated hypotheses per drinking outcome:

Hypothesis 1a. Based on past research suggesting a positive correlation between PTSS and drinking outcomes (Angkaw et al., 2015; Brown \& Wolfe, 1994; Sayer et al., 2010), we anticipated a positive association between PTSS experienced in the past month and alcohol involvement (mediation path $c_{1}{ }^{\prime}$ ).

Hypothesis 1b. Recent evidence has shown PTSD to be related to coping motives (see Lehavot et al., 2014; Simpson et al., 2014). Consistent with these studies, we hypothesized a positive association between PTSS and coping motives (mediation path 
$a_{1}$ ), such that higher past month PTSS would be associated with higher levels of selfreported coping motives for drinking.

Hypothesis 1c. That coping motives correlate with quantity and frequency of alcohol use has been established among adolescents (Cooper, 1994), college students (Kuntsche et al., 2005), and adults (Crutzen \& Kuntsche, 2013). However, alcohol use motivated for this purpose is perhaps most often implicated with negative consequences associated with drinking (Cooper et al., 1995; Cooper, Kuntsche, Levitt, Barber, \& Wolf, in press). Thus, we anticipated coping motives would be positively related to both quantity and frequency of past month alcohol intake (e.g., drinking days, binge drinking), as well as drinking problems (mediation path $b_{1}{ }^{\prime}$ ).

Hypothesis 2a-f. The hypothesized models (see Figure 3.0) specified three pathways through which social support acted as a moderator, (1) the $a$ path from PTSS to drinking to cope motives, suggesting the indirect effect of PTSS on alcohol involvement (quantity, frequency, problems) through motives is conditional on one's level of perceived support, (2) the $b$ path from coping motives to alcohol involvement, and (3) the direct effect from PTSS to drinking outcomes (c path). Consistent with past research and theory, we hypothesized perceptions that one is cared for and supported by friends and family would $(2 a, 2 b)$ reduce the motivation to engage in other compensatory strategies to cope with negative events (e.g., drinking; $a_{2}$ path), (2c, $\left.2 \mathrm{~d}\right)$ diminish the strength of association between coping motives and alcohol involvement 
( $b_{2}$ path), and $(2 e, 2 f)$ buffer or reduce the likelihood that one would engage in harmful health behaviors such as alcohol use ( $c_{2}^{\prime}$ path) (Beattie \& Longabaugh, 1997).

\section{Method}

\section{Participants}

A total of 509 service members consented to participate in the study between April 2014 and November 2015. Ninety seven participants (19.1\%) were excluded from analyses as they reported that they did not consume alcohol during the past 30 days. The final sample of 412 consisted of $84 \%$ men ( 340 men, 67 women) with an average age of 38.7 ( $S D=9.4$ years), a majority of which were married $(69.3 \%)$ and non-Hispanic White (84.1\%). Most participants were recently separated from the military $(81.2 \%$ recently separated vs. $18.8 \%$ active duty) and had been in the military for an average of 12.4 years $(S D=8.3)$. Approximately half $(51 \%)$ of participants reported their most recent military component as being with the National Guard or Reserves. A majority of those who most recently served in an active duty capacity were in the Navy (19\%), Army (11\%), Marines (10\%), or some other branch (9\%). Importantly, past month drinkers included in this study did not differ from non-drinkers on any of these demographic characteristics, nor did they differ in their reported PTSS $(\mathrm{t}(506)=0.2, p=.857)$ or number of combat experiences $(\mathrm{t}(496)=0.6, p=.563)$.

\section{Measures and Procedure}

Baseline Assessment. Data were collected as part of the Study for Employment Retention of Veterans (SERVe), a randomized controlled trial designed to improve 
supports for separated service members and reservists in the workplace. Study team members approached public and private sector organizations throughout the state of Oregon to participate in the training. Fliers and study information were then sent to all participating company employees via email inviting all service members to participate in the evaluation of the training. Interested service members completed a brief online screener to determine eligibility for the study. Those who were employed at least parttime ( $\geq 20$ hours/week) at a participating organization who were also either (a) serving in the National Guard or Reserve components or (b) had recently separated (no earlier that December 31, 2001) from the U.S. armed forces were eligible to participate in the study. The decision to include post-9/11 service members was deliberate. Although it was anticipated that the study training would yield benefits for service members from other conflict eras as well (e.g., Vietnam era, Gulf War era), study aims were to evaluate the effectiveness and impact of such trainings on the health, well-being, and performance of those more recently involved in the military.

After consenting to participate in the study, participants completed a comprehensive baseline assessment during their non-work time using Qualtrics, an online survey tool. The baseline survey took about 45 minutes to complete. Participants responded to a variety of questions about their work, health, family life, and experiences while in the military, and were offered a $\$ 25$ reloadable gift card for completing the baseline survey. 
PCL-M. The four-item PTSD Checklist-Military version (PCL-M; Bliese et al., 2008) was used to assess PTSS. Relative to the PCL-C for civilian populations (Weathers et al., 1993), the PCL-M captures symptoms directly associated with experiences while serving in the military, and has been widely used and validated for use in the military. Using a 5point Likert scale (1 not at all to 5 extremely) participants reported how bothered they had been in the past month by their symptoms. Items included "repeated disturbing memories, thoughts, or images of the stressful experience," "Having physical reactions (like heart pounding, trouble breathing, sweating) when something reminded you of the stressful experience," "Avoiding activities or situations because they reminded you of the stressful experience," and "Having difficulty concentrating" $(\alpha=.92)$.

\section{Alcohol}

Alcohol use. Alcohol use was assessed using three items capturing quantity and frequency of use during the past month (30 days). Participants were shown a graphic that informed them of the definition of a "standard drink" (International Center for Alcohol Policies, 1998) and answered items about the number of (a) days they drank alcohol in the past month, (b) drinks consumed on the average drinking day, and (c) a dichotomous (yes/no) variable indicating whether the participant binge drank based on the highest number of drinks consumed. Based on the National Institute on Alcohol Abuse and Alcoholism (NIAAA) guidelines, men who reported drinking five (5) or more drinks on a single occasion in the past month were considered binge drinkers, (4 or more drinks for women). 
Alcohol problems. ${ }^{1}$ Alcohol problems were estimated based on the Alcohol Use Disorders Identification Test (AUDIT; Saunders, Aasland, Babor, De La Fuente, \& Grant, 1993). The 10-item AUDIT has been demonstrated as a reliable and valid assessment of high quantity and frequency of alcohol use, problematic use and dependence symptoms, and consequences of use in the past year (Allen, Litten, Fertig, \& Babor, 1997).

Coping motives. Drinking to cope motives were assessed using three items taken from the Drinking Motives Questionnaire-Short Form (DMQ-R-SF; Kuntsche \& Kuntsche, 2009). Participants were instructed to think about all the times they drank alcohol and report how often they drank for each of the twelve reasons using a 5-point Likert scale (1 almost never/never to 5 almost always/always). Example items include because it helps you when you feel depressed or nervous, to cheer up when you're in a bad mood, and to forget about your problems $(\alpha=.89)$.

Social support. Perceived social support from friends and family was assessed using 14 items taken from the Perceived Social Support Scale (Procidano \& Heller, 1983); seven items represented support from friends $(\alpha=.92)$ and seven represented support from family $(\alpha=.81)$. Response options were adapted for this study where participants reported their level of agreement with the provided statements using a 1 to 5 scale (Strongly disagree to strongly agree). Example items include my friends (family) give me the moral support I need; I rely on my friends (family) for emotional support; my friends (family) are sensitive to my personal needs. 
Covariates. All models controlled for participant age, race, and gender, as well as current military status (National Guard/Reserves vs. separated). Past research has shown younger individuals more strongly endorse motives for drinking relative to older individuals (Maisto et al., 1999). Similarly, men may be more likely to consume alcohol to cope with stress, and in greater quantities (Nolen-Hoeksema, 2004; Park \& Levenson, 2002). Models predicting alcohol problems similarly controlled for quantity and frequency of alcohol use. Models also adjusted for the personality dimension of neuroticism (N; Donnellan, Oswald, Baird, \& Lucas, 2006), which is characterized by high levels of negative emotionality (David \& Suls, 1999). Past research has linked N with alcohol involvement, but argued that this association can be explained in large part through the endorsement of drinking to cope motivations by those higher in $\mathrm{N}$ (Cooper, Agocha, \& Sheldon, 2000; Kuntsche, von Fischer, \& Gmel, 2008; Stewart \& Devine, 2000).

Finally, given its strong association with PTSS and drinking, combat exposure (i.e., events and stressors one might experience while deployed) was included in statistical models as a covariate. Combat exposure was assessed using a 27 -item checklist where participants indicated how many times each type of event occurred during their most recent deployment from 0 to 5 or more times (Hoge et al., 2004). Consistent with prior research, binary indicators of whether an event was experienced ( 1 Yes 0 No) were summed to generate a composite variable representing the total number of exposures experienced while deployed (Cabrera et al., 2007; Guyker et al., 
2013). Sixty five percent of participants reported multiple exposures $(M=6.46, S D=$ 6.93), with knowing someone who had been seriously injured or killed (52.1\%), receiving incoming artillery, rocket, or mortar fire (48.5\%), and seeing dead bodies or human remains (44.2\%) being the most commonly reported experiences.

\section{Analyses}

Tests of Non-Independence. Because service members were recruited to participate in this study through their respective organizations $(N=36)$, it is possible that alcohol use patterns may be more similar among coworkers within the same organization (Ames, Grube, \& Moore, 2000; Frone, 2014). Intraclass correlations (ICCs) were calculated for each of the primary study outcomes (e.g., drink days, average drinks/day, binging, and alcohol problems), with large ICC values being indicative of between groups differences and a violation of non-independence assumptions (Tabachnik \& Fidell, 2007). Using a conservative ICC cutoff of .10 (Hox, 2002) results indicated no assumptions were violated (ICCs ranged from .001 - .005). In the interest of parsimony, all analyses proceeded using a single level model.

Conditional Process Analysis. ${ }^{2}$ For this study, we were interested in whether drinking to cope motives comprise the mechanism through which PTSS is related to greater alcohol use and problems, and the extent to which these associations are conditional on one's level of perceived support from family and friends. To achieve this, eight conditional process models (Hayes, 2013) were estimated, one for each alcoholrelated outcome (i.e., past year alcohol problems, past month frequency and average 
quantity of drinking, and past month binge drinking). Using Hayes (2013) PROCESS

model 59 (see Figure 3.0), bias-corrected bootstrapping with 5,000 samples were used to estimate the $95 \%$ confidence intervals for the conditional indirect effects.

To examine the unique moderating role of support, models predicting support from family controlled for support from friends, and vice versa. All variables involved in tests of moderation were centered on their respective means (Tabachnick \& Fidell, 2007). Significant interactions were probed by examining simple effects in concert with recommendations of Aiken and West (1991) for high (+1 SD), moderate $(M)$ and low (-1 SD) levels of social support.

\section{Results}

\section{Descriptive Statistics}

Table 3.0 provides descriptive information and intercorrelations for study variables. Most participants had been deployed either overseas or domestically at least once since September, 2001 (82\%), and were deployed for an average of 17 months (SD $=15.0)$. Roughly $18 \%$ met the proposed criteria for probable PTSD of $12+$ provided in Bliese and colleagues (2008) $(M=7.70, S D=4.16)$. On average, participants drank on approximately ten occasions during the past 30 days $(S D=8.70)$, consuming just over two standard drinks per day $(M=2.13, S D=1.65)$. The average AUDIT score was 5.73 $(S D=4.63)$, with twenty-six percent $(N=108)$ meeting criteria for problematic drinking based on an AUDIT score of 8+. Additionally, forty-three percent ( $N=178)$ of participants reported at least one binge drinking episode during the past 30 days. 
Current reservists did not differ from those who were separated in their level of PTSS, endorsement of coping motives, or any alcohol outcomes ( $p s>.05)$.

Indirect and Conditional Indirect Effects ${ }^{3}$

Perceived Support from Friends. Because PROCESS does not explicitly model the $c$ pathway from the predictor to outcome, ordinary least squares regression (OLS) was used to examine the direct association between PTSS and alcohol involvement (results not shown). In partial support of hypothesis 1a, results revealed that when controlling for age, race, gender, neuroticism, and combat exposure, PTSS was significantly and positively related to alcohol consumption variables (drink days, average drinks/day, alcohol problems, and likelihood of binging). However, PTSS was not related to alcohol problems when also controlling for drinking days and average drinks $(b=.25$, $p=.13)$

Conditional process analyses were then conducted examining the moderating role of perceived support from friends (FRIENDS) on the indirect effect of PTSS on alcohol involvement through drinking to cope motives. As shown in Table 3.1, PTSS was positively related to drinking to cope motives (a pathway) in all models, suggesting that participants who experienced greater PTSS in the past month endorsed higher levels of drinking to cope motives (hypothesis $1 \mathrm{~b}$ ). Such motives were associated with higher past year alcohol problems $(b=1.40, p<.001)$, greater self-reported drinking days $(b=$ $3.73, p<.001)$, and greater number of drinks consumed on average $(b=.65, p<.001)$. In addition, those endorsing greater coping motives were at significantly higher odds of 
binging within the past month $(b=1.23, p<.001)$, thus confirming hypothesis $1 \mathrm{c}$ for all models. In all instances, the direct effect of PTSS on alcohol involvement outcomes was nonsignificant $(p>.05)$ when accounting for coping motives, demonstrating full mediation of the association between PTSS and alcohol problems, drinking days, average drinks consumed, and likelihood of binging respectively.

Hypothesis 2 a was supported for the model predicting alcohol problems. First, a significant interaction was observed between PTSS and FRIENDS predicting drinking to cope motives $(b=-.08, p<.05)$. Importantly, this interaction was significant when controlling for both number of drinking days and average number of drinks consumed. Simple slopes tests revealed the effect of PTSS on coping motives to be significant for those perceiving lower $(b=.25, t(385)=5.39, p<.001)$, average $(b=.19, t(385)=4.18, p$ $<.001)$, and higher levels of FRIENDS $(b=.12, t(385)=2.18, p<.05)$. As shown in Figure 3.1, participants who reported lower levels of PTSS endorsed lower levels of coping motives. Although higher levels of PTSS were positively associated with coping motives, these associations were weaker among those who perceived greater support from their friends. Consistent with this evidence, there was a significant conditional indirect effect for PTSS on alcohol problems through coping motives where the indirect effect was significantly different from zero at low $(a b=.33,95 \% \mathrm{BC} \mathrm{Cl}:[.15 ; .65])$ and average $(a b=$ $.26,95 \% \mathrm{BC} \mathrm{Cl}:[.10 ; .49])$ levels of FRIENDS, but not at high levels of FRIENDS ( $a b=.18$, 95\% BC Cl: $[-.01 ; .50])$. 
Interaction effects were marginal for models predicting drinking days, average drinks consumed, and binge drinking ( $b=-.08, p=.06$ in each model). For these models, simple slope tests indicated that although the effect was significant and positive at all levels of FRIENDS, the effect of PTSS on coping motives became weaker as FRIENDS increased ( $b=.33, .27$, and .20 respectively). Formal estimation of conditional indirect effects showed the indirect effects of past month PTSS on drinking days, average drinks consumed, and likelihood of binge drinking through coping motives remained significantly different from zero irrespective of one's level of FRIENDS.

No conditional indirect effects were observed for associations where FRIENDS moderated coping motive-alcohol involvement associations, and no conditional direct effects were observed for any outcomes. Thus, hypotheses $2 c$ and $2 e$ were not supported.

Perceived Support from Family. Similar to the previous models, PROCESS analyses examining the moderating role of perceived support from family (FAMILY) revealed PTSS to be positively and significantly related to drinking to cope motives ( $a$ pathway), further supporting hypothesis $1 \mathrm{~b}$. Consistent with hypothesis $1 \mathrm{c}$, such motives were associated with higher past year alcohol problems $(b=1.42, p<.001)$, greater self-reported drinking days $(b=3.66, p<.001)$, and greater number of drinks consumed, on average $(b=.71, p<.001)$. Finally, those endorsing greater coping motives were at significantly higher odds of binging within the past month $(b=1.31, p<$ $.001)$. 
Unlike previous models, no significant interactions were observed between PTSS and FAMILY predicting coping motives (hypothesis $2 \mathrm{~b}$ ). In contrast, the interaction between coping motives and FAMILY predicting average level of consumption was statistically significant $(b=.34, p<.01)$ (hypothesis $2 d)$. Simple slopes tests revealed the positive association between coping motives and average level of consumption to be significant for those perceiving low $(b=.45, t(387)=4.03, p<.001)$, average $(b=.71$, $t(387)=7.06, p<.001)$, and high levels of FAMILY $(b=.96, t(387)=6.55, p<.001)$. As shown in Figure 3.2, across levels of FAMILY, participants generally reported higher average levels of drinking as coping motives increased. However, this association was strongest among those with the highest level of FAMILY. A similar interaction was observed between coping motives and FAMILY predicting likelihood of binging, although the effect was marginal $(b=.35, p=.08)$.

Examination of conditional indirect effects showed that in all instances the indirect effect of past month PTSS on alcohol outcomes through drinking to cope motives remained significantly different from zero regardless of one's level of FAMILY (see Table 3.1). No conditional direct effects were observed for any outcomes. Thus, hypothesis $2 \mathrm{f}$ was not supported.

\section{Discussion}

Military service members face numerous social, behavioral, mental and physical health challenges as they alternate between military and civilian roles, or reintegrate into their roles as civilians post-separation. Among these concerns are heavy alcohol 
use and symptoms of posttraumatic stress disorder, issues which have strong implications for combat readiness and performance in the field among those still serving (Ames, Cunradi, Moore, \& Stern, 2007; Fisher, Hoffman, Austin-Lane, \& Kao, 2000). Yet, despite the high prevalence of hazardous drinking and PTSD and associated symptoms among service members, most do not report these problems. Indeed, roughly $74 \%$ of our current sample met standardized criteria for being low risk for alcohol abuse and problems according to the AUDIT, and the average level of PTSS reported was below diagnostic thresholds. Results highlight that many who share similar experiences while in the military drink in ways that are less problematic. Importantly, however, this does not diminish the significant need to alleviate the burden of problematic drinking in the military. Forty three percent of our sample of past month drinkers reported at least one binge drinking episode in the past month, a rate substantially higher than the population average of $25 \%$ of (young) adults over 18 who binge drank in the past month (SAMHSA, 2014). Thus, it remains imperative to examine ways in which mental health symptoms resulting from military experiences influence or co-occur with potentially problematic drinking behaviors, and to identify the underlying reasons which motivate or drive these behaviors, as these reasons represent unique determinants of drinking behavior.

The present study represents an important step toward a more integrated understanding of PTSS and alcohol use among military personnel. Recent research efforts have provided preliminary evidence demonstrating the utility of applying 
theoretical models of motivated drinking to military populations, yet have relied almost exclusively on clinical samples of individuals receiving some form of treatment of problematic alcohol use (McDevitt-Murphy et al., 2015a) or comorbid PTSD and alcohol use disorder (Lehavot et al., 2014; Simpson et al., 2014). A strength of our study is its use of a community sample of separated service members as well as currently serving members of the National Guard or Reserves who are currently employed, thus far an underrepresented group in comparison. By extending our recruitment strategy beyond those presenting with pathologic symptoms, our study likely includes a more diverse array of experiences and may be more representative of the general population of post9/11 military service members. More than one in four service members screened positive for alcohol problems in this study, which closely reflects prevalence levels of problem alcohol use observed in other studies of non-dependent service members (e.g., Bray et al., 2013; McDevitt-Murphy et al., 2010;; Wilk et al., 2010). Furthermore, our non-clinical sample of (mostly) moderate drinkers potentially avoids ceiling effects, which could explain inconsistent patterns of results in previous alcohol literature among treatment-seeking service members (e.g., Lehavot et al., 2014).

Our findings demonstrate that high rates of concomitant PTSS and problematic alcohol use commonly observed in military populations (Heltemmes et al., 2014; IOM, 2014; Thomas et al., 2010) can be at least partially explained using theoretical models of motivated drinking (Cooper, 1994). Recent research efforts have examined the interplay of mental and behavioral health among service members by applying similar 
models, however our study adopted a more explanatory approach and addresses substantively different questions from those mentioned previously. For example, McDevitt-Murphy and colleagues (2015a) looked strictly at correlations and mean differences in endorsement of motives between those screening positive and negative for PTSD, whereas others have explored motives as individual difference factors which moderate PTSD-drinking associations (Lehavot et al., 2014; Simpson et al., 2014). While these studies were able to identify for whom these associations were meaningful, none attempted to determine the explanatory role of drinking to cope motives, or to examine the potential conditional nature of these associations by identifying factors such as social support which may buffer or attenuate these associations.

Indeed, the pattern of results suggest the association between PTSS and alcohol involvement (i.e., drinking days, average number of drinks consumed, alcohol problems, and likelihood of binging) is indirect through its association with coping motives. Importantly, direct effects of PTSS on alcohol involvement were non-significant when controlling for coping motives. Such evidence is consistent with motivational perspectives which argue that motives represent a final explanatory pathway through which emotional antecedents influence drinking behavior and consequences (Cooper et al., 1992; Cox \& Klinger, 1988).

Moreover, social influences in the form of perceived support were shown to affect both the reasons why one might drink and also one's resultant behavior. Although conditional indirect effect hypotheses were not supported for some alcohol 
use outcomes in this study, our results provide evidence that perceptions of support from one's close friends (but not family) significantly moderate the association between PTSS and endorsement of coping motives for models predicting alcohol problems (i.e., one's total score on the AUDIT). Consistent with buffering hypotheses (Cohen \& Wills, 1985), perceptions that one is valued and cared for by their close friends was shown to significantly moderate the association between PTSS and drinking to cope motives. Although service members who reported experiencing high levels of PTSS within the past month generally endorsed greater coping motives, this association was weakest among those who perceived they were supported and could rely on their friend in times of need. This finding is particularly relevant given the strong links between coping motives and alcohol problems established in the literature (Cooper et al., in press; Cooper et al., 1992; Kuntsche et al., 2005). Furthermore, the indirect effect of PTSS on alcohol problems through coping motives was conditional on one's level of perceived support from friends. That is, the indirect effect was weaker as support increased, and nonsignificant among those perceiving higher (+ 1 SD) levels of support.

Similar trends were observed when predicting alcohol consumption (quantity, frequency, and likelihood of binging). Although indirect effects of PTSS on alcohol consumption through coping motives weakened at higher levels, indirect effects remained significant at all levels of perceived support from friends. Taken together, these findings highlight close friends as an important influence on service member drinking behavior, particularly potential consequences which may arise from more 
problematic forms of drinking such as drinking to cope. This finding may have implications for well-being and quality of life outcomes beyond alcohol involvement. For example, a recent study by Angkaw and colleagues (2015) suggested alcohol problems (but not level of consumption) mediated associations between PTSS and mental health-related quality of life among OEF/OIF veterans (see also McDevittMurphy et al., 2010). Future research should examine the extent to which these factors influence quality of life outcomes, as inclusion of coping motives and perceptions of support into this larger process may better elucidate how PTSD and related symptoms adversely impact the mental and physical health of service members and critical social resources which may mitigate such deficits.

In contrast, perceptions of support from one's family were shown to moderate associations between coping motives and alcohol involvement, specifically one's average level of consumption. At lower levels of coping motives, those perceiving higher levels of support from family reported the lowest levels of drinking. This effect crossed over, where at higher levels of coping motives, drinking was highest among those perceiving high levels of support from family. Similar effects were observed for the association between coping motives and likelihood of binging, although the effect did not meet traditional statistical significance standards. Within the context of motivated drinking, it appears that perceived support from friends but not family is more influential in determining whether individuals more strongly endorse coping motives for drinking when experiencing PTSS whereas perceived support from family may be more 
influential in determining the strength of the relationship between motives and resultant drinking behavior.

Such results seem to run counter to expectations given the breadth of evidence suggesting various forms of social support are beneficial for reducing certain harmful health behaviors, such as alcohol use (McCreary \& Sadava, 1998; Mohr, Averna, Kenny, \& Del Boca, 2001), yet attempts at providing support are not uniformly effective, and the type of counsel one receives may actually serve to promote negative health behaviors or exacerbate mental health symptoms (Cohen \& LeMay, 2007; Gros et al., 2016; Helgeson, 2003; Russell et al., 2016). A recent study by Gros and colleagues (2016) found evidence that perceptions of support were positively related to number of drinking days over the past 60 days among service members seeking treatment for comorbid PTSD and SUD. They partially attributed this effect to service members seeking support and drinking as a means of accruing social rewards and resources. Consistent with this view, for some, particularly those who are motivated to drink as a means of alleviating stress, alcohol use and support from close others, in this case family, may be confounded. That is, service members experiencing PTSS who endorse motivations to drink to cope may still perceive high levels of support from their families; however the form of support being provided may include activities which involve drinking. Although an investigation of this possibility is beyond the scope of the current investigation, future research should attempt to further delineate the support process by examining the type of support being provided. 
This evidence is not meant to suggest that support from family is in a way detrimental or hazardous for health behavior. Given the cross-sectional nature of this study, it is not possible to discern the temporal ordering of this process. A plausible alternative to this interpretation is that service members who endorse high levels of coping motives may indeed be more likely to drink at higher rates and also engage in binge drinking. In response, families of service members may be increasing their provision of support as a result. In this way, drinking to cope with stress or PTSS may act as a signal to family members that additional support is needed. Indeed, although perceived support from family was not directly related to consumption or alcohol problems (nor was support from friends), it was negatively related to coping motives. To the extent that family members are attuned and responsive to why service members are drinking, such supports may yield positive benefits (i.e., reductions in quantity of use and binging) over time.

\section{Limitations and Future Directions}

As the current study is cross-sectional in nature, causality and directionality cannot be definitively established. However, associations between PTSS, coping motives, and alcohol involvement are consistent with pathways specified in past research and theory where mental health symptoms, including PTSS precede alcohol involvement (Angkaw et al., 2015; Hien et al., 2010), and alcohol use motivations act as a proximal mechanism through which such experiences are related to alcohol involvement (Cooper et al., 1995; Simons et al., 2005). Additionally, recent research has 
shown that PTSS among service members may vary from day to day (Possemato et al., 2015; Simpson et al., 2014) and even within days (Gaher et al., 2014). Despite the strengths of the present study with respect to sample, design, and analytical rigor, by examining associations between PTSS, drinking motives, and alcohol involvement crosssectionally, important substantive information may have been lost. Future research should attempt to examine interrelatedness among PTSS and other symptoms of stress, support processes, and motivated drinking as they unfold in daily life, as daily, withinperson assessments capture psychologically distinct processes from those at the between-person level (Kenny, Kashy, \& Bolger, 1998).

The inclusion of actively serving National Guard and Reservists (NGR) along with separated military from various service branches may limit the generalizability of the present investigation when compared to literature assessing only actively serving or separated military service members. However, as argued previously, our sample did not differ in terms of prevalence of likely PTSD or alcohol problems. Furthermore, consistent with growing literature on the impact of military service on NGRs (e.g., Griffith, 2010; Kehle et al., 2012; Milliken et al., 2007), those who served most recently in the National Guard or Reserves in our study did not differ significantly in their alcohol involvement from those serving in other branches. NGRs face unique challenges leading up to, during, and following deployments which may leave them more susceptible to adverse post-deployment risks and consequences relative to traditional active duty military (Adler et al., 2011; Jacobsen et al., 2008; Milliken et al., 2007). By adopting a more 
inclusive approach, our study design addresses concerns raised by authors such as Sayer and colleagues (2010) who argued that current research practices with respect to the military have often neglected service members from branches other than the Army, particularly NGRs. Further, to our knowledge, this is the first study of its kind to explore the role of PTSS, support processes, and alcohol involvement in a sample of separated service members and reservists employed in a civilian role.

\section{Opportunities for Intervention}

Prior research has shown roughly $40 \%$ of service members report experiencing one or more mental health problems following deployment (e.g., PTSD, depression, heavy alcohol use), however these rates may underestimate the true prevalence of PTSD when one considers the stigma which military culture holds with respect to mental illness (see Britt, 2007). Less than half of those in need report seeking assistance (Burnett-Zeigler et al., 2011; Gorman et al., 2011; Hoge et al., 2004). Although an exploration of treatment-seeking behavior is beyond the scope of the present study, evidence indicates service members are finding alternative means of coping with deployment stress, often-times through increases in drinking behavior. Thus, more specific, tailored interventions are required which are designed to fit an individual's

needs. For example, brief interventions (e.g., McDevitt-Murphy, Williams, Murphy, Monahan, \& Bracken-Minor, 2015b) where education and feedback on one's mental health and substance use is provided in primary care settings may attenuate stigma concerns and provide service members with strategies based on their unique 
experience. Indeed researchers and practitioners have argued the most efficacious interventions are those which address both mental health symptoms (e.g., PTSS) in concert with behavioral health (Hien et al., 2010). As drinking motives represent a "final pathway" (Cox \& Klinger, 1988) through which stress and mental health concerns can influence alcohol involvement, incorporation of modules which account for individual reasons for drinking, such as drinking to cope into treatment regimens would be beneficial (Jacobson et al., 2008; Norman et al., 2014).

In addition to mental health and alcohol-related concerns which produce a heavy individual and societal burden, Sayer and colleagues (2010) identified social relationships (e.g., confiding in others, keeping nonmilitary friendships) as among the major self-reported concerns facing service members as they reintegrate into civilian roles. Although researchers have identified support, cohesion, and effective leadership among unit members as important for well-being (Bliese, 2006; Bliese, Adler, \& Castro, 2011), interventions designed to improve trust, communication, and effective supports among nonmilitary friends and family have been lacking. As such, improved practices with an emphasis on relationship building for service members and their families would likely improve reintegration outcomes greatly.

\section{Conclusion}

The present study suggests that well-established links between PTSS and alcohol involvement observed in military populations can be at least partially explained by applying a theoretical model of drinking motivations. Such motives have tremendous 
utility for intervention efforts designed to reduce harmful drinking behavior among service members. In addition, this study further reinforces the importance of social relationships in influencing health behavior among service members. Future research should attempt to further disentangle contextual factors which influence support effectiveness, including the source of support. 


\section{Footnotes}

${ }^{1}$ To avoid multicollinearity concerns with alcohol use items, an adapted AUDIT score was computed for all PROCESS models based on standardized scoring procedures for items 4-10. Revised AUDIT scores excluded items assessing alcohol consumption (see Appendix II).

${ }^{2}$ Although study hypotheses imply directionality of effects based on evidence from prior research with military populations (e.g., Hien et al., 2010; Kehle et al., 2012; Norman et al., 2014), these analyses are cross-sectional. Therefore it is not possible to establish temporal precedence among study constructs (i.e., Does PTSS precede alcohol use, or is it that alcohol use predicts PTSS?).

${ }^{3}$ Effects of study covariates (age, race, gender, military status, neuroticism, and combat exposure) are not reported. In all PROCESS models, only age was associated with coping motives and drinking outcomes. Younger service members were more likely to endorse past month coping motives, higher average levels of drinking and alcohol problems, and a higher likelihood of binging. Conversely, older participants reported more drinking days in the past month, on average. 


\section{References}

Adler, D. A, Possemato, K., Mavandadi, S., Lerner, D., Chang, H., Klaus, J., ... Oslin, D. W. (2011). Psychiatric status and work performance of veterans of Operations Enduring Freedom and Iraqi Freedom. Psychiatric Services, 62(1), 39-46. doi:10.1176/appi.ps.62.1.39

Aiken, L. S., \& West, S. G. (1991). Multiple regression: Testing and interpreting interactions. Newbury Park, CA: Sage.

Allen, J. P., Litten, R. Z., Fertig, J. B., \& Babor, T. (1997). A review of research on the Alcohol Use Disorders Identification Test (AUDIT). Alcoholism, Clinical and Experimental Research, 21(4), 613-619

American Psychiatric Association. (2013) Diagnostic and statistical manual of mental disorders, (5th ed.). Washington, DC: Author.

Ames, G. M., Cunradi, C. B., Moore, R. S., \& Stern, P. (2007). Military culture and drinking behavior among U.S. Navy careerists. Journal of Studies on Alcohol and Drugs, 68(3), 336-44. Retrieved from http://www.ncbi.nlm.nih.gov/pubmed/17446972

Ames, G. M., Grube, J. W., \& Moore, R. S. (2000). Social control and workplace drinking norms: a comparison of two organizational cultures. Journal of Studies on Alcohol, 61(2), 203-219. 
Angkaw, A.C., Haller, M., Pittman, J.O.E., Nunnink, S.E., Norman, S.B., .. \& Baker, D.G. (2015). Alcohol-related consequences mediating PTSD symptoms and mental health-related quality of life in OEF/OIF combat veterans. Military Medicine, 180, $670-674$.

Baron, R. M., \& Kenny, D. A. (1986). The moderator-mediator variable distinction in social psychological research: Conceptual, strategic, and statistical considerations. Journal of Personality and Social Psychology, 51(6), 1173-1182.

Beattie, M.C., \& Longabaugh, R. (1997). Interpersonal factors and post-treatment drinking and subjective wellbeing, Addiction, 92, 1507-1521.

Bliese, P.D. (2006). Social climates: Drivers of soldier well-being and resilience. In A.B. Adler, C.A. Castro, \& T.W. Britt (Eds.) Military life: The Psychology of Serving in Peace and Combat: Vol. 2. Operational Stress (pp. 213-234). Westport, CT: Praeger.

Bliese, P. D., Adler, A.B., \& Castro, C. A. (2011). Research-based preventive mental health care strategies in the military. In A.B. Adler, P.D. Bliese, \& C.A. Castro (Eds.) Deployment Psychology: Evidence-Based Strategies to Promote Mental Health in the Military (pp. 103-124). Washington, DC: American Psychological Association. 
Bliese, P. D., Wright, K. M., Adler, A. B., Cabrera, O., Castro, C. A., \& Hoge, C. W. (2008). Validating the primary care posttraumatic stress disorder screen and the posttraumatic stress disorder checklist with soldiers returning from combat. Journal of Consulting and Clinical Psychology, 76(2), 272-81. doi:10.1037/0022$006 X .76 .2 .272$

Bray, R. M., Brown, J. M., \& Williams, J. (2013). Trends in binge and heavy drinking, alcohol-related problems, and combat exposure in the U.S. Military. Substance Use \& Misuse, 48, 799-810. doi:10.3109/10826084.2013.796990

Britt, T. W., Greene-Shortridge, T. M., \& Castro, C. A. (2007). The stigma of mental health problems in the military. Military Medicine, 172(2), 157-61. Retrieved from http://www.ncbi.nlm.nih.gov/pubmed/17357770

Brown, P. J., \& Wolfe, J. (1994). Substance abuse and post-traumatic stress disorder comorbidity. Drug and Alcohol Dependence 35, 51-59.

Burnett-Zeigler, I., Ilgen, M., Valenstein, M., Zivin, K., Gorman, L., Blow, A. J., ... Chermack, S. (2011). Prevalence and correlates of alcohol misuse among returning Afghanistan and Iraq Veterans. Addictive Behaviors, 36, 801-806.

Cabrera, O. A., Hoge, C. W., Bliese, P. D., Castro, C. A., \& Messer, S. C. (2007). Childhood adversity and combat as predictors of depression and post-traumatic stress in deployed troops. American Journal of Preventive Medicine, 33, 77-82.

Cohen, S., \& Wills, T. A. (1985). Stress, social support, and the buffering hypothesis. Psychological Bulletin, 98(2), 310-357. 
Conger, J. J. (1956). Alcoholism: Theory, problem and challenge: II. Reinforcement theory and the dynamics of alcoholism. Quarterly Journal of Studies on Alcohol, 17, 296-305.

Cooper, M. L. (1994). Motivations for alcohol use among adolescents: Development and validation of a four-factor model. Psychological Assessment, 6, 117-128.

Cooper, M. L., Agocha, V. B., \& Sheldon, M. S. (2000). A motivation perspective on risky behaviors: The role of personality and affect regulatory processes. Journal of Personality, 68, 1059-1088.

Cooper, M. L., Frone, M. R., Russell, M., \& Mudar, P. (1995). Drinking to regulate positive and negative emotions: A motivational model of alcohol use. Journal of Personality and Social Psychology, 69, 990-1005.

Cooper, M. L., Kuntsche, E., Levitt, A., Barber, L., \& Wolf, S. (in press). A motivational perspective on substance use: Review of theory and research. In K. J. Sher (Ed.), Oxford Handbook of Substance Use Disorders. New York, NY: Oxford University Press.

Cooper, M. L., Russell, M., \& George, W. H. (1988). Coping, expectancies, and alcohol abuse: A test of social learning formulations. Journal of Abnormal Psychology, $97(2), 218-230$.

Cooper, M. L., Russell, M., Skinner, J. B., Frone, M. R., \& Mudar, P. (1992). Stress and alcohol use: Moderating effects of gender, coping, and alcohol expectancies. Journal of Abnormal Psychology, 101, 139- 152. 
Cox, M., \& Klinger, E. (1988). A motivational model of alcohol use. Journal of Abnormal Psychology, 97, 168-180.

Crutzen, R., \& Kuntsche, E. (2013). Validation of the four-dimensional structure of drinking motives among adults. European Addiction Research, 19(4), 222-226. doi:10.1159/000345457

Cucciare, M.A., Darrow, M., \& Weingardt, K.R. (2011). Characterizing binge drinking among U.S., military veterans receiving a brief alcohol intervention. Addictive Behaviors, 36, 362-367.

Dakof, G. A, \& Taylor, S. E. (1990). Victims' perceptions of social support: what is helpful from whom? Journal of Personality and Social Psychology, 58(1), 80-89. doi:10.1037/0022-3514.58.1.80

David, J. P., \& Suls, J. (1999). Coping efforts in daily life: Role of big five traits and problems appraisals. Journal of Personality, 67, 265-294.

Donnellan, M.B., Oswald, F.L., Baird, B.M., \& Lucas, R.E. (2006). The mini-IPIP scales: Tiny-yet-effective measures of the big five factors of personality. Psychological Assessment, 18(2), 192-203.

Fisher, C. A., Hoffman, K. J., Austin-Lane, J., \& Kao, T. (2000). The relationship between heavy alcohol use and work productivity loss in active duty military personnel: A secondary analysis of the 1995 Department of Defense Worldwide Survey. Military Medicine, 5, 355-361. 
Frone, M. R. (2014). Perceived Physical Availability of Alcohol at Work and Workplace Alcohol Use and Impairment : Testing a Structural Model. Psychology of Addictive Behaviors. doi:10.1037/a0037785

Gaher, R. M., Simons, J. S., Hahn, A. M., Hofman, N. L., Hansen, J., \& Buchkoski, J. (2014). An Experience Sampling Study of PTSD and Alcohol-Related Problems. Psychology of Addictive Behaviors, 28, 1013-1025.

Gorman, L. a, Blow, A. J., Ames, B. D., \& Reed, P. L. (2011). National Guard families after combat: mental health, use of mental health services, and perceived treatment barriers. Psychiatric Services, 62(1), 28-34. doi:10.1176/appi.ps.62.1.28

Greeley, J., \& Oei, T. (1999). Alcohol and tension reduction. In K. E. Leonard \& H. T. Blane (Eds.), Psychological theories of drinking and alcoholism (2nd ed.) (pp. 14-53). New York, NY: Guilford Press.

Gros, D.F., Flanagan, J.C., Korte, K.J., Mills, A.C., Brady, K.T., \& Back, S.E. (2016). Relations among social support, PTSD symptoms, and substance use in veterans. Psychology of Addictive Behaviors. doi: http://dx.doi.org/10.1037/adb0000205

Griffith, J. (2010). Citizens coping as Soldiers: A review of deployment stress symptoms among Reservists. Military Psychology, 22, 176-206.

Guyker, W. M., Donnelly, K., Donnelly, J. P., Dunnam, M., Warner, G. C., Kittleson, J., Bradshaw, C. B., ... Meier, S. T. (2013). Dimensionality, reliability, and validity of the Combat Experiences Scale. Military Medicine, 178, 377-384. 
Hammer, L.B. (2013). NG/RC Reintegration in the civilian workforce: The critical role of supervisors. Symposia presented at the 2013 annual meeting of the American Psychological Association, Honolulu, HI.

Hayes, A. F. (2013). Introduction to mediation, moderation, and conditional process analysis: A regression-based approach. New York, NY: The Guilford Press.

Hien, D. A., Jiang, H., Campbell, A. N. C., Hu, M., Miele, G. M., Cohen, L., ... \& Nunes, E. V. (2010). Do treatment improvements in PTSD severity affect substance use outcomes? A secondary analysis from a randomized clinical trial in NIDA's Clinical Trials Network. American Journal of Psychiatry, 167, 95-101.

Hoge, C. W., Castro, C. A., Messer, S. C., McGurk, D., Cotting, D. I., \& Koffman, R. L. (2004). Combat duty in Iraq and Afghanistan, mental health problems, and barriers to care. The New England Journal of Medicine, 351(1), 13-22.

Hoge, C. W., Terhakopian, A., Castro, C. A., Messer, S. C., \& Engel, C. C. (2007). Association of posttraumatic stress disorder with somatic symptoms, health care visits, and absenteeism among Iraq war veterans. The American Journal of Psychiatry, 164(1), 150-153. doi:10.1176/appi.ajp.164.1.150

Hox, J. (2002). Multilevel analysis techniques and applications. Mahwah, NJ: Lawrence Erlbaum Associates. 
International Center for Alcohol Policies. (1998). What is a standard drink? Retrieved from http://www.icap.org/portals/0/download/all_pdfs/ICAP_Reports_English/report 5.pdf.

IOM (Institute of Medicine). (2012). Substance use disorders in the U.S. Armed Forces. Washington, DC: The National Academies Press.

IOM (Institute of Medicine). 2014. Treatment for posttraumatic stress disorder in military and veteran populations: Final assessment. Washington, DC: The National Academies Press.

Jacobson, I. G., Ryan, M. A. K., Hooper, T. I., Smith, T. C., Amoroso, P. J., Boyko, E. J., ... Bell, N. S. (2008). Alcohol Use and Alcohol-Related Problems Before and After Military Combat Deployment. JAMA, 300(6), 663-675.

Kehle, S. M., Ferrier-Auerbach, A. G., Meis, L. A., Arbisi, P. A., Erbes, C. R., \& Polusny, M. A. (2012). Predictors of postdeployment alcohol use disorders in National Guard soldiers deployed to Operation Iraqi Freedom. Psychology of Addictive Behaviors, 26(1), 42-50.

Kenny, D., Kashy, D., \& Bolger, N. (1998). Data analysis in social psychology. In D. Gilbert, S. Fiske, \& G. Linzey (Eds.), Handbook of Social Psychology (4 ${ }^{\text {th }}$ ed., pp. 233-265). New York: McGraw-Hill. 
King, L. A, King, D. W., Fairbank, J. A, Keane, T. M., \& Adams, G. A. (1998). Resiliencerecovery factors in post-traumatic stress disorder among female and male Vietnam veterans: hardiness, postwar social support, and additional stressful life events. Journal of Personality and Social Psychology, 74(2), 420-34.

King, D. W., King, L. A, Foy, D. W., Keane, T. M., \& Fairbank, J. A. (1999). Posttraumatic stress disorder in a national sample of female and male Vietnam veterans: risk factors, war-zone stressors, and resilience-recovery variables. Journal of Abnormal Psychology, 108(1), 164-170. doi:10.1037/0021-843X.108.1.164

Kuntsche, E., Knibbe, R., Gmel, G., \& Engels, R. (2005). Why do young people drink? A review of drinking motives. Clinical Psychology Review, 25(7), 841-861.

Kuntsche, E., \& Kuntsche, S. (2009). Development and validation of the Drinking Motive Questionnaire Revised Short Form (DMQ-R SF). Journal of Clinical Child and Adolescent Psychology, 38(6), 899-908. doi:10.1080/15374410903258967

Kuntsche, E., von Fischer, M., \& Gmel, G. (2008). Personality factors and alcohol use: A mediator analysis of drinking motives. Personality and Individual Differences, 45(8), 796-800. doi:10.1016/j.paid.2008.08.009

Lehavot, K., Stappenbeck, C. A, Luterek, J. A, Kaysen, D., \& Simpson, T. L. (2014). Gender differences in relationships among PTSD severity, drinking motives, and alcohol use in a comorbid alcohol dependence and PTSD sample. Psychology of Addictive Behaviors, 28(1), 42-52. doi:10.1037/a0032266 
Maguen, S., Vogt, D.S., King, L.A., King, D.W., \& Litz, B.T. (2006). Posttraumatic growth among Gulf War I veterans: The predictive role of deployment-related experiences and background characteristics. Journal of Loss and Trauma: International Perspectives on Stress \& Coping, 11, 373-388.

Maisto, S. A, Carey, K. B., \& Bradizza, C. M. (1999). Social Learning Theory. In K. E. Leonard \& H. T. Blane (Eds.), Psychological theories of drinking and alcoholism (2nd ed.) (pp. 106-163). New York, NY: Guilford Press.

McCarthy, E. P. E., \& Petrakis, I. (2010). Epidemiology and management of alcohol dependence in individuals with post-traumatic stress disorder. CNS Drugs, 24(12), 997-1007. doi:10.2165/11539710-000000000-00000

McCreary, D. R., \& Sadava, S. W. (1998). Stress, drinking, and the adverse consequences of drinking in two samples of young adults. Psychology of Addictive Behaviors, $12,247-261$.

McDevitt-Murphy, M. E., Williams, J. L., Bracken, K. L., Fields, J. A., Monahan, C. J., \& Murphy, J. G. (2010). PTSD symptoms, hazardous drinking, and health functioning among U.S. OEF/OIF veterans presenting to primary care. Journal of Traumatic Stress, 23, 108-111.

McDevitt-Murphy, M. E., Fields, J. A., Monahan, C. J., \& Bracken, K. L. (2015a). Drinking motives among heavy-drinking veterans with and without posttraumatic stress disorder. Addiction Research \& Theory, 23, 148-155. 
McDevitt-Murphy, M. E., Williams, J. L., Murphy, J. G., Monahan, C. J., \& Bracken-Miller, K. L. (2015b). Brief intervention to reduce hazardous drinking and enhance coping among OEF/OIF/OND veterans. Professional Psychology: Research and practice, $46,83-89$.

McNally, R. J. (2003). Progress and controversy in the study of posttraumatic stress disorder. Annual Review of Psychology, 54, 229-252.

Milliken, C. S., Auchterlonie, J. L., \& Hoge, C. W. (2007). Longitudinal assessment of mental health problems among active and reserve component soldiers returning from the Iraq war. JAMA : The Journal of the American Medical Association, 298(18), 2141-2148.

Mohr, C. D., Averna, S., Kenny, D. a, \& Del Boca, F. K. (2001). “Getting by (or getting high) with a little help from my friends": an examination of adult alcoholics' friendships. Journal of Studies on Alcohol, 62, 637-645.

Nillni, Y. I., Gradus, J. L., Gutner, C. A., Luciano, M. T., Shipherd, J. C., \& Street, A. E. (2014). Deployment Stressors and Physical Health Among OEF / OIF Veterans : The Role of PTSD. Health Psychology, 33(11), 1281-1287.

Nolen-Hoeksema, S. (2004). Gender differences in risk factors and consequences for alcohol use and problems. Clinical Psychology Review, 24(8), 981-1010. doi:10.1016/j.cpr.2004.08.003 
Norman, S. B., Schmied, E., \& Larson, G. E. (2014). Predictors of Continued Problem Drinking and Substance Use Following Military Discharge. Journal of Studies on Alcohol and Drugs, 75, 557-566.

Park, C. L.,\& Levenson, M. R. (2002).Drinking to cope among college students: Prevalence, problems and coping processes. Journal of Studies on Alcohol, 63(4), 486-497.

Pietrzak, R. H., Johnson, D. C., Goldstein, M. B., Malley, J. C., Rivers, A. J., Morgan, C. A., \& Southwick, S. M. (2010). Psychosocial buffers of traumatic stress, depressive symptoms, and psychosocial difficulties in veterans of Operations Enduring Freedom and Iraqi Freedom: The role of resilience, unit support, and postdeployment social support. Journal of Affective Disorders, 120, 188-192. doi:10.1016/j.jad.2009.04.015

Possemato, K., Maisto, S. A, Wade, M., Barrie, K., McKenzie, S., Lantinga, L. J., \& Ouimette, P. (2015). Ecological Momentary Assessment of PTSD symptoms and alcohol use in combat veterans. Psychology of Addictive Behaviors, 29, 894-905. doi:10.1037/adb0000129

Procidano, M.E., \& Heller, K. (1983). Measures of perceived social support from friends and family: Three validation studies. American Journal of Community Psychology, 11, 1-24. 
Russell, D. W., Benedek, D. M., Naifeh, J. A., Fullerton, C. S., Benevides, N., Ursano, R. J., .. . Cacioppo, J. T. (2016). Social Support and Mental Health Outcomes Among U.S. Army Special Operations Personnel. Military Psychology. doi: 10.1037/mil0000114

Russell, D. W., Russell, C. A., Riviere, L. a, Thomas, J. L., Wilk, J. E., \& Bliese, P. D. (2014). Changes in alcohol use after traumatic experiences: the impact of combat on Army National Guardsmen. Drug and Alcohol Dependence, 139, 47-52. doi:10.1016/j.drugalcdep.2014.03.004

Sadava, S. W., \& Pak, A. W. (1993). Stress-related problem drinking and alcohol problems: A longitudinal study and extension of Marlatt's model. Canadian Journal of Behavioural Science, 25(3), 446-464. doi:10.1037/h0078841.

Saunders, J., Aasland, O., Babor, T., De La Fuente, J., \& Grant, M. (1993). Development of the Alcohol Use Disorders Test (AUDIT): WHO collaborative project on early detection of persons with harmful alcohol consumption-II. Addiction, 88, 791804.

Sayer, N. A., Noorbaloochi, S., Frazier, P., Carlson, K., Gravely, A., \& Murdoch, M. (2010). Reintegration Problems and Treatment Interests Among Iraq and Afghanistan Combat Veterans Receiving VA Medical Care. Psychiatric Services, 61(6), 589597.

Sayette, M. A. (2000). Does drinking reduce stress? Alcohol Research \& Health, 23, 250255. 
Schnurr, P. P., \& Spiro III, A. (1999). Combat exposure, Posttraumatic Stress Disorder symptoms, and health behaviors as predictors of self-reported physical health in older veterans. The Journal of Nervous and Mental Disease, 187(6), 353-359.

Simons, J. S., Gaher, R. M., Correia, C. J., Hansen, C. L., \& Christopher, M. S. (2005). An affective-motivational model of marijuana and alcohol problems among college students. Psychology of Addictive Behaviors, 19(3), 326-34.

Simpson, T. L., Stappenbeck, C. A, Luterek, J. A, Lehavot, K., \& Kaysen, D. L. (2014). Drinking motives moderate daily relationships between PTSD symptoms and alcohol use. Journal of Abnormal Psychology, 123(1), 237- 247.

Stahre, M. A., Brewer, R. D., Fonseca, V. P., \& Naimi, T. S. (2009). Binge drinking among U.S. active-duty military personnel. American Journal of Preventive Medicine, 36, 208-217.

Stewart, S. H., \& Devine, H. (2000). Relations between personality and drinking motives in young adults. Personality and Individual Differences, 29, 495-511.

Substance Abuse and Mental Health Services Administration (SAMHSA). 2014 National Survey on Drug Use and Health (NSDUH). Table 2.46B-Alcohol use, binge alcohol use, and heavy alcohol use in the past month among persons aged 18 or older, by demographic characteristics: Percentages, 2013 and 2014. Available at: http://www. samhsa.gov/data/sites/default/files/NSDUH-DetTabs2014/NSDUHDetTabs2014.htm\#tab2-46b

Sundin, J., Jones, N., Greenberg, N., Rona, R. J., Hotopf, M., Wessely, S., \& Fear, N. T. (2010). Mental health among commando, airborne and other UK infantry 
personnel. Occupational Medicine (Oxford, England), 60(7), 552-9. doi:10.1093/occmed/kqq129

Tabachnick, B.G., \& Fidell, L.S. (2007). Using Multivariate Statistics, $5^{\text {th }}$ ed. Boston: Pearson.

Tanielian, T., and L. H. Jaycox (Eds.). 2008. Invisible wounds of war: Psychological and cognitive injuries, their consequences, and services to assist recovery. Santa Monica, CA: RAND Corporation.

Thomas, J. L., Wilk, J. E., Riviere, L. A., Mcgurk, D., Castro, C. A., \& Hoge, C. W. (2010). Prevalence of Mental Health Problems and Functional Impairment Among Active Component and National Guard Soldiers 3 and 12 Months Following Combat in Iraq. Archives of General Psychiatry, 67(6), 614-623.

Uchino, B., Cacioppo, J., Kiecolt-Glaser, J., \& Anonymous. (1996). The relationship between social support and physiological processes: A review with emphasis on underlying mechanisms and implications for health. Psychological Bulletin, 119(3), $488-531$.

Weathers, F. W., Litz, B. T., Herman, D. S., Huska, J. A., \& Keane, T. M. (1993). The PTSD Checklist (PCL): Reliability, validity, and diagnostic utility. Paper presented at the annual meeting of the International Society for Traumatic Stress Studies, San Antonio, TX 
Wilcox, S. (2010). Social relationships and PTSD symptomatology in combat veterans.

Psychological Trauma: Theory, Research, Practice, and Policy, 2(3), 175-182.

doi:10.1037/a0019062 


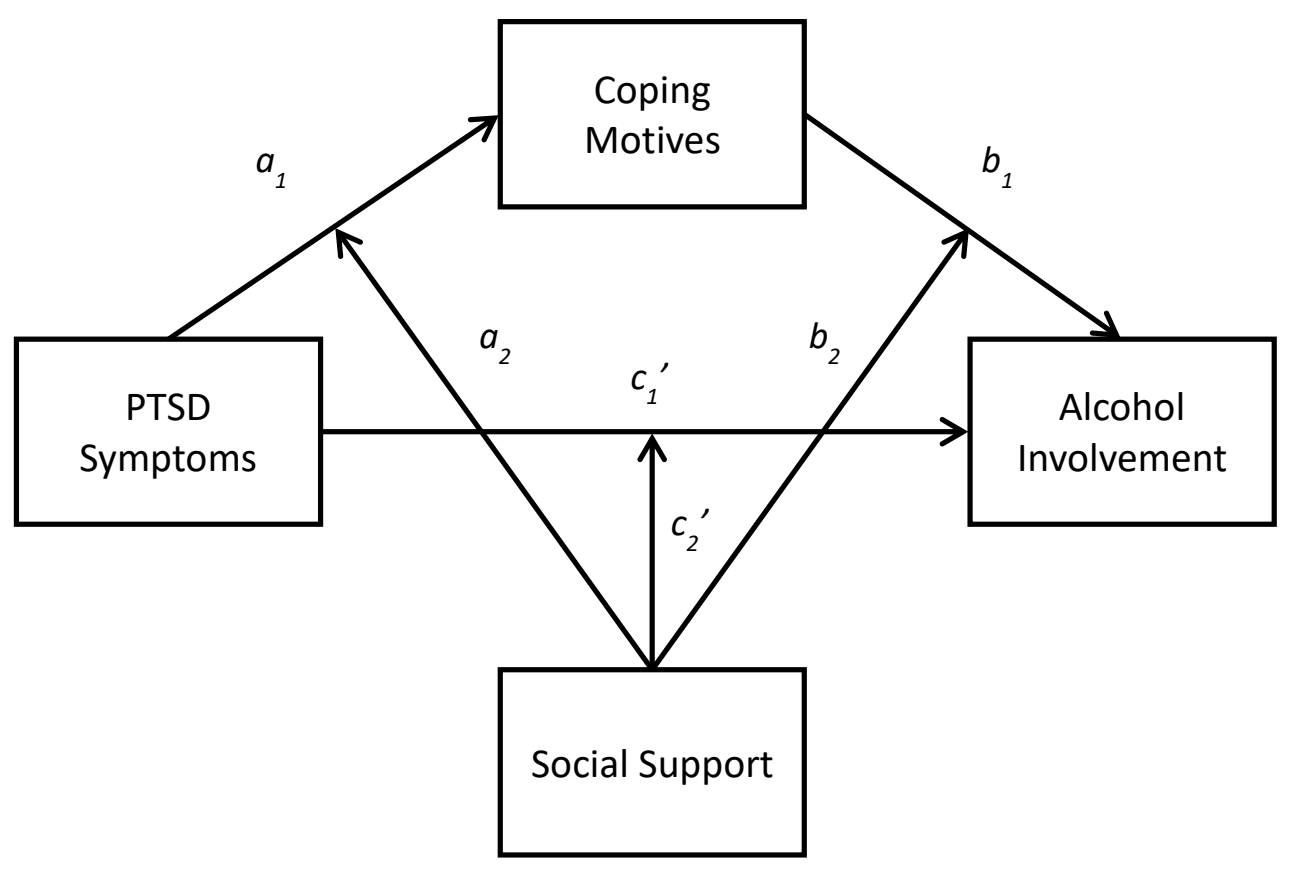

Figure 3.0. Hypothesized model of the conditional indirect effect of PTSD symptoms on alcohol involvement through drinking to cope motives. 


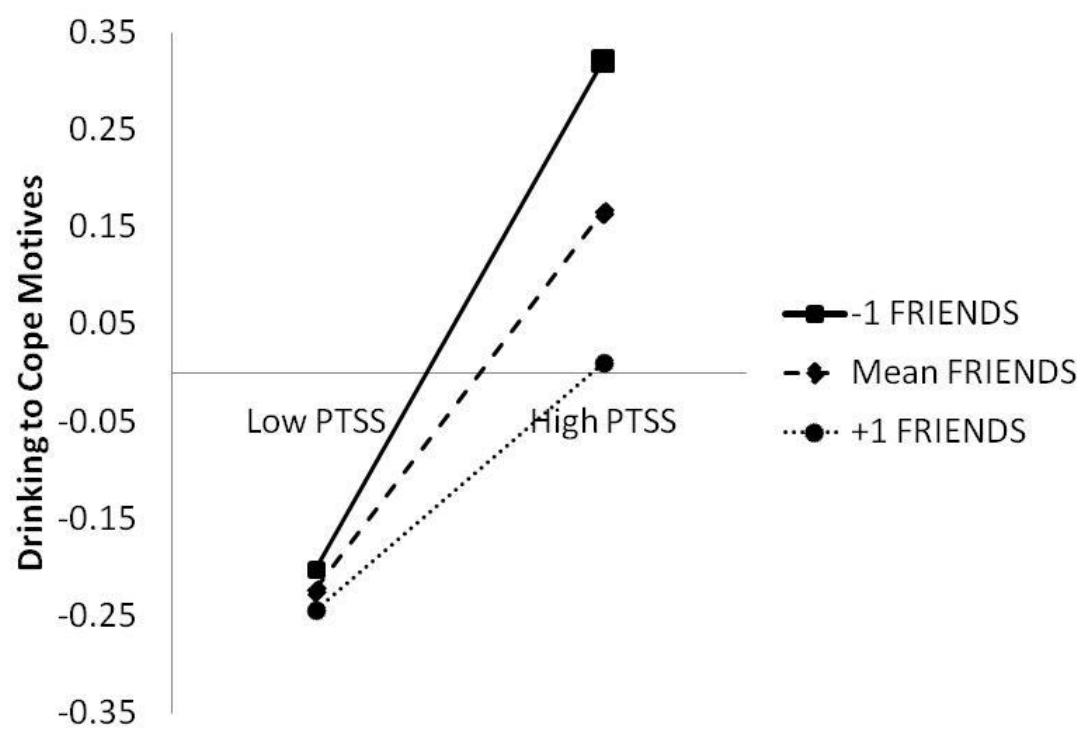

Figure 3.1. Moderating effect of perceived support from friends (FRIENDS) on PTSD symptom-coping motives association - AUDIT model.

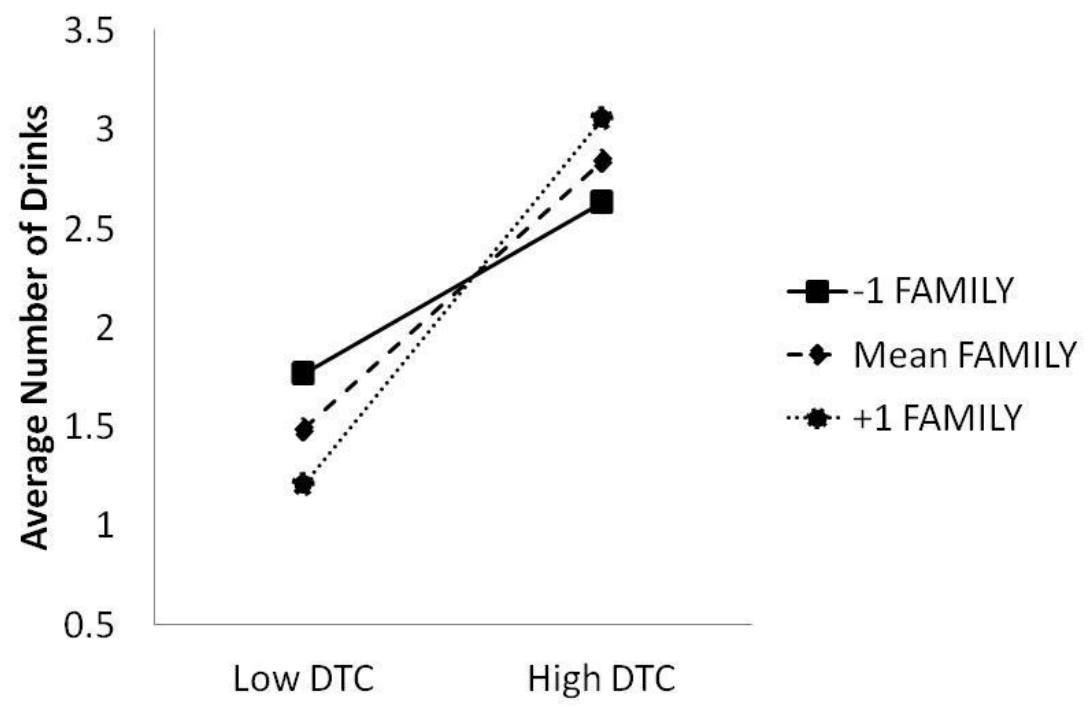

Figure 3.2. Moderating effect of perceived support from family (FAMILY) on the association between coping motives (DTC) and average number of drinks consumed. 
Table 3.0. Descriptive statistics and intercorrelations

\begin{tabular}{|c|c|c|c|c|c|c|c|c|c|c|c|c|c|c|c|c|c|c|}
\hline & $M$ & SD & Min & Max & 1 & 2 & 3 & 4 & 5 & 6 & 7 & 8 & 9 & 10 & 11 & 12 & 13 & 14 \\
\hline 1. PTSS & 7.70 & 4.16 & 4 & 20 & 1.00 & & & & & & & & & & & & & \\
\hline 2. DTC & 1.68 & 0.96 & 1 & 5 & $.45^{*}$ & 1.00 & & & & & & & & & & & & \\
\hline 3. FRIEND & 3.47 & 0.82 & 1 & 5 & $-.28 *$ & $-.30 *$ & 1.00 & & & & & & & & & & & \\
\hline 4. FAMILY & 3.66 & 0.76 & 1 & 5 & $-.20 *$ & $-.30 *$ & $.38^{*}$ & 1.00 & & & & & & & & & & \\
\hline 5. ALCDAY & 9.73 & 8.70 & 1 & 30 & $.13+$ & $.33^{*}$ & -.04 & -.07 & 1.00 & & & & & & & & & \\
\hline 6. AVEALC & 2.13 & 1.65 & 0 & 10 & $.20 *$ & $.35^{*}$ & -.04 & -.06 & $.18^{*}$ & 1.00 & & & & & & & & \\
\hline 7. AUDIT & 5.72 & 4.63 & 1 & 29 & $.27 *$ & $.59 *$ & $-.13^{*}$ & $-.15^{*}$ & $.49 *$ & $.60 *$ & 1.00 & & & & & & & \\
\hline 8. BINGE & - & - & - & - & $.19 *$ & $.41^{*}$ & $-.12^{*}$ & $-.10 \dagger$ & $.35 *$ & $.51^{*}$ & $.62 *$ & 1.00 & & & & & & \\
\hline 9. AGE & 38.70 & 9.41 & 21 & 68 & $-.16 *$ & $-.13^{*}$ & .04 & -.02 & $.12+$ & $-.13 *$ & $-.16 *$ & $-.19 *$ & 1.00 & & & & & \\
\hline 10. RACE & - & - & - & - & .01 & .03 & -.02 & .02 & .04 & .03 & .06 & .05 & -.08 & 1.00 & & & & \\
\hline 11. GENDER & - & - & - & - & .01 & .10 & .07 & -.02 & -.03 & -.06 & -.01 & -.04 & -.03 & .01 & 1.00 & & & \\
\hline 12. MILSTAT & - & - & - & - & .07 & .04 & $-.15^{*}$ & -.07 & .08 & .04 & .07 & .05 & .01 & .06 & .03 & 1.00 & & \\
\hline 13. $\mathrm{N}$ & 2.81 & 0.93 & 1 & 5 & $.58^{*}$ & $.42 *$ & $-.35^{*}$ & $-.29 *$ & .05 & $.13^{*}$ & $.20 *$ & $.16 \dagger$ & $-.12 \dagger$ & -.01 & .05 & $.12 \dagger$ & 1.00 & \\
\hline 14. CES & 5.76 & 6.86 & 0 & 27 & $.41^{*}$ & $.17^{*}$ & $-.13^{*}$ & -.07 & .05 & .09 & $.13+$ & .09 & $-.12 \dagger$ & -.03 & $-.21^{3}$ & .05 & $.23^{*}$ & 1.00 \\
\hline
\end{tabular}

Variables in bold represent primary study variables and outcomes; PTSS = Posttraumatic Stress Symptoms; DTC = drinking to cope motives;

FRIENDS = perceived support from friends; FAMILY = perceived support from family; ALCDAYS = number of drinking days in past month;

AVEALC = average number of drinks consumed per day; AUDIT = total score on 10-item Alcohol Use Disorders Identification Test; BINGE =

binge drank during past 30 days; MILSTAT = military status (current National Guard/Reservist vs. separated); $\mathrm{N}=\mathrm{Neuroticism}$; CES = combat

exposure

* Significant at $p<.01$

+ Significant at $p<.05$ 
Table 3.1. Conditional process analyses of the association between posttraumatic stress symptoms and alcohol involvement

\begin{tabular}{|c|c|c|c|c|c|c|c|c|c|c|c|c|}
\hline \multirow[b]{2}{*}{ Path/Effect } & \multirow[b]{2}{*}{ B } & \multirow[b]{2}{*}{ SE } & \multicolumn{3}{|c|}{ ALCDAYS } & \multicolumn{3}{|c|}{ AVEALC } & \multicolumn{2}{|c|}{ AUDIT } & \multicolumn{2}{|r|}{ BINGE } \\
\hline & & & $95 \mathrm{Cl}$ & $\mathrm{B}$ & SE & $95 \mathrm{Cl}$ & $\mathrm{B}$ & SE & $95 \mathrm{Cl}$ & $\mathrm{B}$ & SE & $95 \mathrm{Cl}$ \\
\hline \multicolumn{13}{|l|}{ Perceived Support: Friends (FR) } \\
\hline$a$ (PTSS-DTC) & $.27^{*}$ & .05 & {$[0.16 ; 0.37]$} & $.27^{*}$ & .05 & {$[0.16 ; 0.37]$} & $.19 *$ & .05 & {$[0.09 ; 0.28]$} & $.27 *$ & .05 & {$[0.16 ; 0.37]$} \\
\hline$b$ (DTC-alcohol) & $3.73^{*}$ & .53 & {$[2.68 ; 4.78]$} & $.65^{*}$ & .10 & {$[0.44 ; 0.85]$} & $1.40^{*}$ & .17 & {$[1.07 ; 1.73]$} & $1.23^{*}$ & .19 & {$[0.86 ; 1.61]$} \\
\hline$c^{\prime}$ (PTSS-alcohol) & .37 & .55 & {$[-0.71 ; 1.45]$} & .16 & .11 & {$[-0.05 ; 0.37]$} & -.04 & .16 & {$[-0.46 ; 0.33]$} & .02 & .16 & {$[-0.29 ; 0.33]$} \\
\hline$a \times b$ (indirect effect) for $-1 S D$ FR & $1.24 *$ & .35 & {$[0.66 ; 2.03]$} & $.19 *$ & .06 & {$[0.09 ; 0.32]$} & $.33^{*}$ & .13 & {$[0.15 ; 0.65]$} & $.36^{*}$ & .13 & {$[0.17 ; 0.62]$} \\
\hline$a \times b$ (indirect effect) for mean FR & $.99 *$ & .30 & {$[0.48 ; 1.63]$} & $.17^{*}$ & .05 & {$[0.09 ; 0.30]$} & $.26^{*}$ & .10 & {$[0.10 ; 0.49]$} & $.33^{*}$ & .10 & {$[0.15 ; 0.54]$} \\
\hline$a \times b$ (indirect effect) for $+1 S D$ FR & $.74^{*}$ & .38 & {$[0.20 ; 1.73]$} & $.15^{*}$ & .07 & {$[0.05 ; 0.31]$} & .18 & .13 & {$[-0.01 ; 0.50]$} & $.28 *$ & .13 & {$[0.08 ; 0.61]$} \\
\hline \multicolumn{13}{|l|}{ Perceived Support: Family (FAM) } \\
\hline$a$ (PTSS-DTC) & $.28^{*}$ & .05 & {$[0.18 ; 0.39]$} & $.28^{*}$ & .05 & {$[0.18 ; 0.39]$} & $.20^{*}$ & .05 & {$[0.10 ; 0.30]$} & $.28^{*}$ & .05 & {$[0.18 ; 0.39]$} \\
\hline$b$ (DTC-alcohol) & $3.66^{*}$ & .53 & {$[2.62 ; 4.71]$} & $.71^{*}$ & .10 & {$[0.51 ; 0.90]$} & $1.42 *$ & .17 & {$[1.09 ; 1.75]$} & $1.31^{*}$ & .20 & {$[0.91 ; 1.70]$} \\
\hline$c^{\prime}$ (PTSS-alcohol) & .25 & .55 & {$[-0.83 ; 1.33]$} & .15 & .10 & {$[-0.05 ; 0.36]$} & -.06 & .16 & {$[-0.37 ; 0.25]$} & .04 & .16 & {$[-0.28 ; 0.35]$} \\
\hline$a \times b$ (indirect effect) for -1SD FAM & $1.02 *$ & .33 & {$[0.49 ; 1.76]$} & $.12^{*}$ & .05 & {$[0.05 ; 0.25]$} & $.25^{*}$ & .12 & {$[0.08 ; 0.54]$} & $.29 *$ & .10 & {$[0.12 ; 0.50]$} \\
\hline$a \times b$ (indirect effect) for mean FAM & $1.03 *$ & .30 & {$[0.51 ; 1.69]$} & $.20^{*}$ & .06 & {$[0.11 ; 0.32]$} & $.29 *$ & .10 & {$[0.12 ; 0.52]$} & $.37^{*}$ & .11 & {$[0.17 ; 0.60]$} \\
\hline$a \times b$ (indirect effect) for $+1 S D$ FAM & $1.04 *$ & .41 & {$[0.39 ; 2.04]$} & $.28 *$ & .09 & {$[0.13 ; 0.47]$} & $.32 *$ & .14 & {$[0.10 ; 0.65]$} & $.45^{*}$ & .17 & {$[0.19 ; 0.82]$} \\
\hline
\end{tabular}

PTSS = Posttraumatic Stress Symptoms; DTC = drinking to cope motives; ALCDAYS = number of drinking days in past month;

AVEALC = average number of drinks consumed per day; AUDIT = total score for adapted, 7-item Alcohol Use Disorders Identification test (AUDIT);

BINGE = binge drank within the past 30 days

* coefficients significant at $p<.001$ 


\title{
CHAPTER IV. MANUSCRIPT III. DRINKING TO COPE WITH JOB STRESS: A DAILY PROCESS INVESTIGATION OF STRESS AND COPING AMONG ACTIVE DUTY AND SEPARATED MILITARY PERSONNEL.
}

\begin{abstract}
High levels of alcohol use are commonplace among members of the U.S. armed forces. Much attention has been devoted to exploring comorbid alcohol abuse and more severe forms of mental illness and symptoms, yet relatively little is known about how separated service members and reservists respond to ongoing stressors such as job stress in their civilian roles. Moreover, some individuals may be more or less vulnerable for engaging in stress-related drinking. Using a daily process design, this study investigated whether on days individuals experience higher levels of job stress (e.g., role overload, interpersonal conflict) they were (a) more likely to drink and (b) report higher levels of alcohol use that evening and the moderating role of drinking to cope motives and more adaptive coping strategies (emotional and instrumental support seeking). All participants were separated service members and reservists who were married or cohabiting with a romantic partner, and were recruited as part of a 32-day sub-study of a randomized-controlled trial designed to improve military support in civilian workplaces. Results revealed male and female service members did not differ in their level or frequency of drinking, and although service members in general drank at rates higher than those reported in civilian populations, in most instances, service members drank significantly less on days they experienced higher levels of job stress. Moderation results revealed a complex pattern of associations whereby daily job stress-evening
\end{abstract}


drinking associations differed depending on the type of stressor experienced, one's level of endorsement of coping motives, and the form of support seeking service members engaged in. Researchers, practitioners, and military command should continue efforts to curb excessive drinking in the military, and be cognizant of the converging pattern of drinking among male and female service members and its implications for their health, fitness, and combat readiness.

Keywords: Occupational stress, alcohol use, drinking to cope, social support, daily diary 


\section{Introduction}

The consumption of alcohol has long played a role in military culture (Jones \& Fear, 2011), and its use remains prevalent among service members (Bray, Brown, \& Williams, 2013; Jacobson et al., 2008; Milliken, Auchterlonie, \&Hoge, 2007; Stahre, Brewer, Fonseca, \& Naimi, 2009). Specifically, rates of binge and heavy drinking among service members remains significantly higher compared to their age and gendermatched non-military counterparts (Bray, Marsden, \& Peterson, 1991). Approximately $43 \%$ of the U.S. armed forces engaged in binge drinking in the past month (Stahre et al., 2009), and roughly $20 \%$ reporting heavy drinking (Bray et al., 2010). Such use among military populations has been linked with a myriad of mental and physical health, social, and occupational consequences (Adler et al., 2011; Bray et al., 2013; Fisher et al., 2000; Heslin et al., 2012). While alcohol may be consumed as a way of "blowing off steam" or having a good time while on leave (Ames, Cunradi, Moore, \& Stern, 2007), researchers and clinicians have focused much of their attention and effort on understanding the use of substances such as alcohol to aid in symptom management for mental health concerns such as posttraumatic stress disorder (PTSD), depression, and anxiety. Yet, despite robust support for the link between trauma-induced stressors and high levels of alcohol use among service members, much less is known about how some groups, such as those who have since separated from the military and reservists, cope with day-today stressors and hassles such as occupational stress in their civilian roles. Such stressors may similarly manifest into physical, mental, or behavioral symptoms of strain 
(Frone, 1999; Mroczek \& Almeida, 2004; Ganster \& Rosen, 2013; Sonnentag \& Frese, 2013), including high levels of alcohol consumption or problems.

Conger's (1956) tension reduction hypothesis posits that a primary drive (motivation) for consuming alcohol is to reduce or avoid aversive stimuli such as stress. Indeed, a recent study of service members participating in a large-scale brief intervention for alcohol use demonstrated that roughly $60 \%$ of service members selfreported consuming alcohol in order to manage stress or symptoms of depression (Cucciare, Darrow, \& Weingardt, 2011). Despite the apparent pervasiveness of this form of drinking, critics of tension reduction models cite it as an overly-simplistic representation for why individuals consume alcohol. Although a wealth of empirical and anecdotal evidence exists pertaining to stress-related drinking, the assumed causal pathway wherein stress leads to increases in drinking behavior is neither robust, nor consistent in the literature (Greeley \& Oei, 1999; Sayette, 2000), particularly when examining occupational stressors (Cooper, Russell, \& Frone, 1990; Frone, 1999; Grunberg, Moore, Anderson-Connolly, \& Greenberg, 1999). Frone (1999) identified several potential explanations for these mixed findings and proposed that researchers should better attend to how individuals respond to varying types of stressors, potential moderators of stress-drinking relationships which better specify who may be more or less likely to engage in such behaviors, and the use of daily diary and other longitudinal methods to better capture how individuals respond to stress in situ. 
As service members return to the civilian workforce, or balance civilian and military work roles, it is critical to identify how daily experiences of occupational stress affect service member health and health behavior. Further, specification of strategies which may mitigate these concerns may similarly be used to better inform prevention and intervention efforts. In concert with the recommendations of Frone (1999), the purpose of the present investigation is three-fold: (1) to examine the role of daily work stressors on subsequent alcohol use, (2) to identify individuals who may be more or less susceptible to stress-drinking patterns through an exploration of motivated drinking, and (3) to determine whether more adaptive or effective coping strategies can be utilized which ameliorate the stress-drinking relationship.

Presently, the predominant method for conducting research of this kind with military populations has been to evaluate cross-sectional self-report surveys measured using a single timepoint, or large scale epidemiological surveys. While a wealth of knowledge has been gleaned from these efforts, such methods are ill-suited to assess the day-to-day fluctuations in stressful experiences which may facilitate or promote alcohol use. On the other hand, ecological momentary assessments (EMA; Stone \& Shiffman, 1994) and other daily process methods which capture these phenomena in context, as they occur are ideally suited for this purpose. Such methods have been used to explore associations between general forms of occupational stress (Butler, Dodge, \& Faurote, 2010; Carney, Armeli, Tennen, Affleck, \& O’Neil, 2000; Liu, Wang, Zhan, \& Shi, 2009), work-family conflict (Wang, Liu, Zhan, \& Shi, 2010) and alcohol use outcomes at 
the daily level. For example, Liu and colleagues (2009) found that relative to those who were less involved, individuals reporting high levels of job involvement (i.e., those whose jobs were central to their self-definition), daily experiences of job stress were associated with greater desire to drink, as well as daily quantity of drinks consumed. Similar effects were found for individuals high in the personality dimension of Neuroticism (Liu et al., 2009).

By capturing stressful experiences and behavior in close proximity to its actual occurrence, daily diaries limit retrospection among participants and provide more accurate information (Bolger, Davis, \& Raffaeli, 2003; Swendsen, Tennen, Carney, Affleck, \& Willard, 2000; Tennen, Affleck, Armeli, \& Carney, 2000). Importantly, these methods also allow for the examination of processes inherent in tension reduction models of drinking (Mohr et al., 2005); that is, do individuals drink more on days when they experience higher levels of stress, patterns which are consistent with drinking to cope. Although widely implemented by psychologists to study interpersonal relationships, emotions, and occupational health, these methods have only recently been applied to the study of military health (see Gaher et al., 2014; Simpson, Stappenbeck, Luterek, Lehavot, \&Kaysen, 2014).

\section{Occupational Stress and Drinking}

In addition to methodological concerns with tension reduction models, problems may arise from the way in which stress is assessed or operationalized (Frone, 1999; McCreary \&Sadava, 1998). Studies relying on general measures of job stress or 
demands have found limited support that stress leads to an increase in alcohol consumption as suggested by tension reduction hypothesis. For example, in their crosssectional investigation of blue and white-collar manufacturing employees, Grunberg and colleagues (1999) found that general job stress was inversely related to quantity and frequency of alcohol consumption, as well as alcohol problems. Others (e.g., Frone, 1999) have called for the adoption of a more nuanced approach which differentiates between varying stressors. To address this concern, the present study examined two distinct types of occupational stressors, role overload and interpersonal conflict at work. Role overload $(\mathrm{RO})$ represents a role-based stressor where the needs and demands of one's job exceed their perceived resources to accomplish their goals. Individuals experiencing RO may have fewer resources to engage in self- or emotion regulatory behaviors when stressed, thus may be less effective in their selection and utilization of coping strategies, and may be more apt to consume alcohol in times of stress. Cross-sectional evidence in support of this proposition was found by Frone (2008) where work overload was related to both quantity and frequency of alcohol consumption during and following the work day. However, slightly different patterns have been observed when examining within-person variation in stress-drinking association. Butler, Dodge, and Faurote (2010) found that daily experiences of heavy job demands and workload were not significantly associated with alcohol use among a sample of college student workers. Similar effects were observed by Carney and colleagues (2000) in that negative experiences at work, particularly feeling time 
pressure or having to work overtime, were unrelated to daily drinking behavior, but were significantly and positively associated with higher desire to drink. They described this finding in a way consistent with conservation of resource theory (COR; Hobfoll, 1989), in that participants wanted to consume alcohol on days they experienced higher levels of demands and pressure, perhaps to relieve stress or unwind, but may have lacked the capacity or resources to do so given those demands. Further work is needed to unpack this complex association and describe how individuals respond to experiences of RO on a daily basis.

Interpersonal conflicts represent some of the most salient and aversive experiences one can encounter (Bolger, DeLongis, Kessler, \& Schilling, 1989), and have been implicated as a potential antecedent of daily alcohol consumption (Armeli, Dehart, Tennen, Todd, \& Affleck, 2007; Mohr et al., 2001; 2005). In their daily process study of community dwelling adults, Mohr and colleagues (2001) demonstrated that on days when they experienced a greater number of negative interpersonal events (e.g., being yelled at, having one's goals impeded by another), individuals reported higher levels of drinking at home, and lower levels of drinking away from home (see also Mohr et al., 2005). Similarly, Armeli and colleagues (2007) found evidence that among those with negative alcohol outcome expectancies, interpersonal conflict during the day was associated with higher levels of drinking later that night. That is, those who were unconcerned with the potential consequences of their drinking were more likely to consume alcohol after experiencing an interpersonal conflict. As interpersonal stressors 
and conflicts may have similar implications for relapse among substance dependent individuals (Larimer, Palmer, \& Marlatt, 1999), it is important to evaluate how service members are managing such experiences on an ongoing basis.

\section{Moderators of Stress-Drinking Associations}

According to Cooper and colleagues' (1992a) Stressor Vulnerability Model, some individuals may be more (or less) likely to regulate negative emotions through the use of alcohol and other substances. Among the individual differences identified in the Stressor Vulnerability Model, two factors are particularly relevant for better delineating how service members respond behaviorally to occupational stress: (1) alcohol use motivations, particularly drinking to cope, and (2) the use of alternative coping resources or styles. Examination of these vulnerability factors may better elucidate who is most likely to exhibit stress-drinking patterns, and under what conditions these patterns are likely to occur.

Drinking to Cope Motives. Motivational perspectives of alcohol use have been used to explain pathways through which stress and emotional experiences influence alcohol involvement, as motives represent one of the most proximal determinants of alcohol involvement (Cooper, 1994; Cooper, Russell, Skinner, \& Windle, 1992b; Cox \& Klinger, 1988; Kuntsche et al., 2005). Although models have specified a number of possible motivations which may influence drinking behavior (e.g., to forget your problems, to socialize with others, to fit in, to have a good time; Cooper, 1994), such 
motives are distinct and unique with respect to the etiology of alcohol use, associated outcomes, and their potential consequences (Cooper, Frone, Russell, \& Mudar, 1995).

As is described within tension reduction hypothesis, individuals who are motivated to drink to cope with stress do so as a form of emotion regulation in an effort to suppress aversive emotional experiences or to achieve a state of diminished awareness (Hull, 1981). This pattern of maladaptive coping is particularly pernicious as alcohol use is continuously reinforced, which over time diminishes one's ability to cope effectively without the use of alcohol, contributing to the development of alcohol dependence (Greeley \& Oei, 1999; Norman, Schmied, \& Larson, 2014). Indeed crosssectional evidence suggests that coping motives are associated with greater problems with alcohol, irrespective of the quantity and frequency with which one actually drinks (Carey \& Correia, 1997; Cooper et al., 1995; Simons, Gaher, Correia, Hansen, \& Christopher, 2005). It stands to reason that individuals who endorse coping motives are more likely exhibit stress-drinking patterns. Conversely, those reporting low levels may be less likely to drink when stressed.

Social Support Coping. McCreary and Sadava (1998) argue that when stressed, in order to resolve the discrepancy between one's current and desired state, the determining factor in one's decision to drink may also be partly attributed to the availability of alternative resources to cope with such demands. Consistent with this perspective, the utilization of certain coping strategies in times of high work stress may offset or buffer motivations to consume alcohol. In particular, social relationships and 
supports from others provide a source of social capital, and represent an important coping resource (DeLongis \& Holtzman, 2005; Luchman \& Gonzalez-Morales, 2013). Support from others support has been shown to directly benefit health and well-being, as well as buffer the deleterious effects of both acute and chronic forms of stress (Cohen \& Wills, 1985; Thoits, 1995), and also alcohol use (Peirce, Frone, Russell, \& Cooper, 1996; Steptoe, Wardle, Pollard, Canaan, \& Davies, 1996). In their 35 day study of Chinese employees, Wang and colleagues (2010) found that the association between daily work-family conflict and drinking was weaker among those who perceived higher levels of support from their family (relative to lower levels).

Along with more general perceptions that one is supported by others, past research and theory suggests support can provide valuable coping resources from which one can draw on in times of stress (Cohen \& Wills, 1985; Carver \& Connor-Smith, 2010). Following stressful events or encounters, individuals may seeking advice or support from others for strategies, tangible resources, or assistance to resolve the situation (i.e., instrumental support). Conversely, individuals may seek comfort, validation, and reassurance from others in an attempt to alter their emotional response to the event, particularly if the event is outside of their control. Emotional and instrumental support coping reflect aspects of emotion and problem-focused coping respectively, and may serve to effectively mitigate the aversive experience associated with stress, thereby reducing the need or desire to consume alcohol. 


\section{Present Study}

As continued attention and resources are devoted toward intervention and prevention efforts to curb problematic drinking among service members (IOM, 2012), a greater accounting of the underlying motivations for drinking, as well the presence or utilization of alternative coping resources (e.g., support seeking behavior) is needed to better understand who is likely to exhibit drinking to cope patterns, and under what conditions drinking to cope patterns are likely to occur. Although preliminary evidence which supports claims that service members readily endorse multiple underlying motives for drinking (see Cucciare et al., 2011), there is a relative paucity of information about individual differences in susceptibility to drinking beyond evaluation of deployment and military-related characteristics (e.g., combat exposure, PTSD).

Until recently, few have examined alcohol use as a form of affect or symptom regulation among military populations (see Lehavot, Stappenbeck, Luterek, Kaysen, \& Simpson, 2014; McDevitt-Muprhy et al., 2015a; Simpson et al., 2014). However, these studies differ from our own in important ways. For example, others have relied on clinical samples of heavy drinkers and individuals receiving treatment for comorbid PTSD and alcohol use disorder (AUD). While Simpson and colleagues found evidence that participants high in coping motives used alcohol to self-medicate with daily PTSD symptoms, McDevitt-Murphy and colleagues' (2015a) cross-sectional study was primarily descriptive in nature, revealing that veterans with PTSD reported higher mean levels of coping-depression and coping-anxiety motives relative to those without PTSD. 
Interestingly, these groups did not differ in their endorsement of other drinking motives. To our knowledge, no studies to date have examined the extent to which lower intensity, transient experiences of increased occupational stress influence subsequent drinking behavior among military service members. Furthermore, we know of no studies which have simultaneously explored alternative coping resources (e.g., emotional and instrumental support coping) as potential buffers of this process. As such, the present investigation represents one of the first to examine these issues among a community sample of service members employed in a civilian role.

The present study investigated the role of person-level drinking motives in determining how daily experiences of job stress predict subsequent drinking behavior. Specifically, using a daily process design, we examined whether the association between service member's daily experiences of role overload and interpersonal conflict and drinking later that evening are contingent on one's endorsement of drinking to cope motives. In addition, we sought to clarify whether these patterns differ based on the use of alternative coping strategies, emotional and instrumental support respectively. To delineate the process through which motivated drinking occurs, we evaluated several models whereby job stressors were used to predict (1) total quantity of daily alcohol consumption and (2) daily decision to drink (yes/no).

Hypotheses. Because we were interested in stressful work experiences which precede drinking temporally, we focused on drinking that occurred in the evening, after work. Prior daily process research with community samples (e.g., Swendsen et al., 2000) 
have shown over $90 \%$ of drinking occurs during this time period. In general, we anticipated individuals would be more likely to drink $\left(\mathrm{H}_{1 \mathrm{a}, 1 \mathrm{~b}}\right)$ and in higher quantities $\left(\mathrm{H}_{1 \mathrm{c}, 1 \mathrm{~d}}\right)$ during the evening on days when individuals experience higher levels of role overload and interpersonal conflict respectively. We anticipated these positive associations would be moderated by drinking to cope motives, such that individuals endorsing higher (but not lower) levels of coping motives would be more likely to drink $\left(\mathrm{H}_{2 a}, 2 \mathrm{~b}\right)$ and in higher quantities $\left(\mathrm{H}_{2 c, 2 d}\right)$ in the evening. Finally, we hypothesized threeway interactions where average use of emotional and instrumental support coping strategies across the study period buffered the impact of coping motives on stressdrinking associations ( $\mathrm{H}_{3 a-h}$; see Figures 4.0-4.3 for conceptual models). Specifically, we hypothesized that the moderating effect of coping motives on work stress-evening drinking associations would be buffered or lessened by the use of emotional and instrumental support seeking strategies. That is, although individuals reporting higher coping motives may be more likely to drink (and in greater quantities) on days they experienced greater work stress (i.e., role overload or interpersonal conflict at work), we hypothesized this association would be weaker among those who reported using higher levels of social support coping (either emotional or instrumental support coping).

\section{Method}

\section{Participants}

As part of a randomized controlled trial designed to improve work environments and supports, actively serving and recently separated service members were recruited 
through organizations throughout Oregon. Interested service members completed an initial brief screener to determine eligibility for the study. Service members who were employed at least part-time ( $\geq 20$ hours/week) with a participating organization and were also either (a) actively drilling or (b) recently separated (no earlier that December 31, 2001) from the U.S. armed forces were eligible to participate in the broader study. Those who were either married or cohabiting with a romantic partner were invited to participate in the complementary 32-day online study of military families; therefore all participants in the present investigation were in a committed relationship at the time they enrolled. Participation in the daily study was entirely voluntary, and a decision to not take part in the daily portion did not adversely affect one's enrollment in the larger study. The decision to include post-9/11 a member of the U.S. armed forces was purposeful. Although it was anticipated that the study training would similarly benefit service members from other conflict eras as well (e.g., Vietnam era, Gulf War era), study aims were to evaluate the effectiveness and impact of such trainings on the health, wellbeing, and performance of active (i.e., those serving in the National Guard and Reserves), and recently separated service members.

A total of 175 service members consented to participate in the 32-day study between August 2014 and November 2015. To effectively examine the association between daytime role overload, interpersonal conflict and evening alcohol use, eleven (11) participants with fewer than two consecutive days of data were excluded from analyses. Forty-nine participants (29\%) reported that they abstained from drinking 
during the 32 days, and were not included in subsequent analyses. Finally, because the present investigation was interested in the association between day-time work stressors on evening consumption, sixteen (16) participants who worked either night shifts or variable shifts and were excluded from analyses, resulting in a total analysis sample of 99 service members. The remaining participants provided valid data for approximately $76 \%$ of study days $\left(M_{\text {days }}=24.2, S D=9.8\right)$, which is similar to compliance rates of other daily diary studies (Butler et al., 2010; Liu et al., 2009; Wang et al., 2010). Our final analyses were restricted to days in which service members reported working, resulting in a final sample of 1,452 person days $\left(M_{\text {days }}=17.8, S D=5.0\right){ }^{1}$

Participants were 39 years old, on average ( $S D=9.0$ years), male ( 85 men, 14 women), non-Hispanic white (86.9\%), and married (85.7\% married). Most participants were recently separated from the military ( $80.6 \%$ separated vs. $19.4 \%$ active duty) and had been in the military for over twelve years $(S D=8.0)$ on average.

\section{Procedure}

Baseline Assessment. Prior to consenting, participants completed the baseline assessment and were sent a comprehensive guide broadly describing the goals and procedures of the daily study. This guide contained detailed descriptions on the types of questions participants could expect, and how to access and fill out the daily surveys. Research staff were on call during data collection to respond to any participant issues that may have come up. 
Daily Assessment. Each day for 32 days, participants would log in to the secure survey between 5:00 PM and 11:00 PM using a unique username and. An alternative survey was created to accommodate individuals working non-regular shifts (e.g., night shift, swing shift) and was available between 5:00 AM and 11:00 AM. Participants were instructed to complete surveys after work but before bed time and responded to questions about their work day, health behaviors, and relationship with their partner. Parents were also asked questions about daily experiences with their children. For their continued participation in the study, service members could earn up to $\$ 90$, including weekly bonuses; parents could earn an additional \$25 per couple.

Participants had the opportunity to make up 3 interviews they may have missed throughout the study period, a majority of whom did not use their allotted missed day surveys $\left(M_{\text {days }}=1.2, S D=1.01\right)$. A total of 114 missed day surveys were included in analyses, most of which were completed close to their initial intake date $\left(M_{\text {lag time }}=2.2\right.$ days, $S D=3.5)$.

\section{Measures}

Person-Level Drinking Motives. Drinking to cope motives were assessed at baseline using the three items taken from the Drinking Motives Questionnaire short form (DMQ-R-SF; Kuntsche \& Kuntsche, 2009). Participants were asked to indicate the extent to which they drink for the following reasons: To cheer up when you're in a bad mood, to forget about your problems, and because it helps you when you feel depressed 
or nervous ( $\alpha=.85$ ) using a 5-point scale (1 never or almost never to 5 always or almost always).

Average Social Support Coping. Support coping was assessed once daily using two items representing instrumental (e.g., I tried to get advice from someone about what to do) and emotional support seeking (e.g., I tried to get emotional support from friends or relatives) from the Brief COPE (Carver, 1997). Participants reported how much they used each strategy using a 5-point scale ( 1 not at all to 5 a lot). These items were aggregated across the reporting period for each period. Thus, emotional and instrumental support coping represented the average level of use of these strategies across the study.

Daily Job Stress. Role overload and interpersonal conflict at work were adapted for daily use from two dimensions of Zohar's (1997) Role Hassles index. Three sample items for role overload include felt under time pressure, had difficulty due to insufficient time; had too much work - too many things to take care of; and had to stay too many extra hours or do inconvenient shift-work schedules. Interpersonal conflict at work was assessed using three items, had to interact with an inconsiderate or disliked person; encountered a lack of cooperation or an inconsideration; and had an argument or confrontation about differing views. Participants reported how disruptive the events were on a 4-point scale (1 not disruptive to 4 very disruptive). Day-level internal consistency for daily job stress variables were computed by selecting three days representing the beginning (day 2), middle (day 13 ), and end (day 22 ) of the study. 
Results revealed acceptable reliabilities of $\alpha$ of $.78, .74$, and .83 for role overload and $.87, .81, .80$ for interpersonal conflicts.

Daily Alcohol Use. Daily alcohol use was assessed for three time-points:

Morning, afternoon, and evening. Two items referring to drinking that occurred today between 12:00 AM - 8:00 AM and today 8:00 AM - 4:00 PM were not analyzed in this study. Participants were also asked about their evening consumption that occurred yesterday between 4:00 PM - 12:00 AM. Framing the question this way prevented a loss of information pertaining to daily experiences which occurred during the survey reporting window. A lead variable was created based on this item which allowed for drinking that occurred during the evening to correspond with work events that occurred earlier that same day. Thus our final analyzable sample consisted of 31 possible reporting days. For each time-point, participants reported how many standard drinks (NIAAA, 2015) they consumed during that time period.

Two sets of models were run using two separate alcohol use outcomes. In concert with past research (e.g., Carney et al., 2000; Grunberg et al., 1999) quantity of alcohol consumed in the evening, following work (4:00 PM - 12:00 AM) was used in one set of analyses using a lead function for reports of drinking that occurred the previous evening. Additionally, given the complex association between occupational stressors and daily quantities of alcohol use observed in other studies, daily experiences of role overload and interpersonal conflict may better predict ones initial decision to drink, particularly among those who are generally motivated to use alcohol to diminish stress 
(Kuntsche et al., 2005). To test this possibility, we generated a dichotomous variable which indicated whether participants drank that evening (0 No, 1 Yes).

Covariates. Statistical models controlled for grand-mean centered age and gender (uncentered) as previous research among military populations have consistently shown that younger, male service members drink considerably more relative to their older and female counterparts (Jacobson et al., 2008; Stahre et al., 2009). As is common in daily process studies, we controlled for the previous night's consumption and day of the week effects as drinking is more likely to occur and in higher quantities on weekend days relative to week days (Armeli, Carney, Tennen, Affleck, \& O’Neil, 2000; Finlay, Ram, Maggs, \& Caldwell, 2012; Mohr et al., 2005). Six day of the week dummy codes were computed with Tuesday as the referent (Mohr et al., 2001; 2005). Additionally, as participants may be more apt to drink prior to non-work days, we controlled for whether participants worked the next day.

\section{Results}

\section{Descriptive Statistics}

Table 4.0 provides descriptive information and inter-correlations for study variables. Participants reported consuming alcohol on more than six evenings during the 32-day study $\left(M_{\text {days }}=6.3, S D=6.0\right)$, drinking an average of 2.15 standard drinks per drinking day $(S D=1.2)$ with the highest levels of consumption occurring on Friday evenings $(M=1.5, S D=1.8)$ and lowest on Monday $(M=.84, S D=1.4)$. On average, men consumed alcohol in higher quantities on drinking days relative to women $\left(M_{M}=2.2\right.$, 
$\left.M_{W}=1.8\right)$, whereas women consumed alcohol more frequently relative to men $\left(M_{M}=\right.$ 6.0, $\left.M_{W}=8.3\right)$. Importantly, however, these differences were not statistically significant $(\mathrm{t}(87)=1.2, p=.218$ and $\mathrm{t}(96)=-1.3, p=.185$ respectively $)$. Men and women also did not differ in their average levels of RO, IPC, or use if IS, however women reported significantly greater use of ES strategies to cope with daily stress $(\mathrm{t}(97)=-2.4, p=.02)$. A vast majority of observations fell between Monday and Friday with less than six (6) percent of workdays occurring on the weekend. Although participants were instructed that surveys were meant to be completed during non-work hours, on 28 occasions (2\%), participants reported that they were still working while completing the survey.

\section{Multilevel Analyses}

Multilevel modeling (MLM) using HLM v7 (Raudenbush \& Bryk, 2002) was used to account for the nested structure of the data where days were nested within individuals. This analytic procedure accounts for nonindependence inherent in daily process studies, and allows for the simultaneous modeling of between- (level 2) and within-person (level 1) processes (Raudenbush \& Bryk, 2002). MLM uses maximum likelihood estimation (MLE) for modeling outcomes with unbalanced or missing data. All level 1 predictors (e.g., role overload and interpersonal conflict) were person-centered and modeled as random effects. In contrast, level 2 moderators (e.g., coping motives, average emotional or instrumental coping) and the continuous covariate age were grand-mean centered, while categorical covariates (e.g., gender) were entered uncentered, and treated as fixed effects. Because emotional and instrumental support 
coping were strongly associated $(b=.48, p<.001)$, the moderating role of these predictors were tested separately. To adjust for overdispersion in the level 1 outcomes using count data, models predicting quantity of evening alcohol use were run using a Poisson model. The drinking day outcome was nonlinear (0 No, 1 Yes), therefore multilevel logistic regression were tested using a Bernoulli model (Tabachnick \& Fidell, 2007, pp. 820), whereby the outcome represented the predicted probability of consuming alcohol that evening.

Multilevel models were run in accordance with the recommendations of Raudenbush and Bryk (2002, pp. 256-278). A preliminary, unconditional (null) model (see equation 1) allowed for the computation of the intraclass correlation (ICC). An ICC of . 45 for evening alcohol use suggested $55 \%$ of the variability in evening drinking was associated with within-person factors.

$$
\operatorname{Drink}_{i t}=\beta_{0 t}+e_{i t}
$$

Level 1 predictors were then entered into the model simultaneously. To examine the association between work stress variables and evening alcohol use, evening alcohol consumption was modeled as a function of person-centered stress (see equation 2). The following equations use RO as an illustration.

$$
\operatorname{Drink}_{i t}=\beta_{0 t}+\beta_{1 t} R O+e_{i t}
$$

Here, Drink represents person i's evening drinking on day $t, \beta_{0}$ represents the predicted number of alcohol consumed for person $i$ when role overload is 0 (at its average) on day 
$t$. The within-person slope for role overload for person $i$ on day $t$ is represented by $\beta_{1}$. Finally, $\mathrm{e}_{\mathrm{it}}$ represents random error.

Next, coping motives (DTC), mean support coping (ESC), and grand-mean centered age were entered simultaneously at level 2, while gender was entered uncentered.

$$
\begin{gathered}
\beta_{0 \mathrm{t}}=\gamma_{00}+\gamma_{01} \text { DTC }+\nu_{02} \text { MeanESC }+\gamma_{03} \text { DTCXESC }+\gamma_{04} \text { Age }+ \\
\gamma_{05} \text { Gender }+u_{0 j} \\
\beta_{1 \mathrm{t}}=\gamma_{10}+\nu_{11} \text { DTC }+\gamma_{12} \text { MeanESC }+\nu_{13} \text { DTCXESC }+\gamma_{14} \text { Age }+ \\
\gamma_{15} \text { Gender }+u_{1 j}
\end{gathered}
$$

As displayed in equation 4 , the coefficient $\gamma_{10}$ represents the average within-person slopes for role overload. To examine individual differences in evening consumption due to drinking to cope motives and average support coping, $\gamma_{01}$ and $\gamma_{02}$ represent the person-level main effects of coping motives and emotional support coping respectively (controlling for grand-mean centered age and gender). The person-level interaction between coping motives support coping is represented by $\mathrm{Y}_{03}$ (controlling for mean age and gender). Cross-level interactions between person-centered role overload and grandmean centered coping motives and support coping are represented by $\gamma_{11}$, and $\gamma_{12}$ respectively, controlling for mean age and gender. Finally, coefficient $\gamma_{31}$ represents the three-way interactions between role overload, coping motive, and support coping. Simple slopes were tested to probe interactions and establish a range of significance for 
significant moderation effects in models predicting level of evening consumption (Aiken \& West, 1991).

Role Overload - Evening Drinking Associations. First, level 1 models were analyzed where evening drinking was regressed on experiences of role overload at work that day (see Equation 2). Contrary to prediction, controlling for day of the week, level of alcohol consumed the previous day, and whether participants worked the following day, daily experiences of role overload were significantly and negatively associated with evening drinking $(b=-.04, p=.03)$, thus, Hypothesis $1_{c}$ was not supported. This effect can be readily interpreted by exponentiating the partial slope $(\exp (-.04)=.96)$. Doing so reveals a one unit increase in daily role overload is associated with a $4 \%$ reduction in evening alcohol consumption. Results from all subsequent models are displayed in Table

\section{1.}

Moderating effects of coping motives and emotional support coping. Next, two models controlling for age and gender were tested to evaluate the moderating role of coping motives and emotional and instrumental support coping respectively. Personlevel drinking to cope motives were positively associated with evening alcohol use $(b=$ $.18, p=.08)$, and moderated the association between role overload and evening drinking, although the effect was marginal (Hypothesis $2_{c}: b=-.04, p=.06$ ). Contrary to prediction, those endorsing stronger coping motives (+1 standard deviation above the mean on coping motives) reported greater drinking on days characterized by lower levels of role overload at work (see Figure 4.4). Simple slopes revealed the positive 
association between coping motives and evening drinking was strongest at lower levels of role overload $(b=.25, p=.02)$, but was nonsignificant at higher levels of overload. This interaction was qualified by a three-way, cross-level interaction with average emotional support coping (Hypothesis $3_{\mathrm{e}}: b=-.13, p=.08$ ), although this effect failed to meet traditional statistical significance standards. As depicted in Figure 4.5, lower levels of drinking were observed on days characterized by high levels of role overload only among those endorsing stronger coping motives and who utilized higher emotional support coping over the 32-day period $(b=-.17, p=.01)$. Those endorsing stronger coping motives but who utilized lower emotional support coping reported higher levels of evening drinking irrespective of their level of daily role overload ( $b=-.01, p=.91$ ).

\section{Moderating effects of coping motives and instrumental support coping.}

Surprisingly, role overload was not significantly associated with daily alcohol use in models examining the moderating role of average instrumental support coping $(b=-.01$, $p=.51)$. However, higher endorsement of coping motives $(b=.21, p=.03)$ and lower average instrumental support coping across the study period $(b=-.48, p<.01)$ were associated with greater daily alcohol use. These associations were qualified by a significant three-way cross-level interaction (Hypothesis $3_{\mathrm{f}}: b=.09, p=.02$ ). As depicted in Figure 4.6 and counter to the effect observed for emotional support, lower levels of drinking were observed on days characterized by high levels of role overload among those endorsing stronger coping motives and lower use of instrumental support coping over the 32-day period $(b=-.07, p<.01)$. Those endorsing stronger coping motives, who 
utilized higher average instrumental support coping reported higher levels of evening drinking on days they experienced greater role overload $(b=.05, p=.10)$.

Interpersonal Conflict at Work - Evening Drinking Associations. The same procedures were used to examine the association between interpersonal conflict experienced at work and evening drinking. Similar to the previous models and contrary to prediction, controlling for day of the week, level of alcohol consumed the previous day, and whether participants worked the following day, daily experiences of interpersonal conflict were significantly and negatively associated with evening drinking $(b=-.04, p=.02)$, Thus Hypothesis $1_{d}$ was not supported. Exponentiating the partial slope $(\exp (-.04)=.96)$ reveals a one unit increase in daily interpersonal conflict is associated with a $4 \%$ reduction in alcohol consumption that evening.

\section{Moderating effects of coping motives and emotional support coping. When}

testing the full model, participants reported only marginal reductions in drinking on days they experienced higher levels of interpersonal conflict at work $(b=-.03, p=.08)$. Person-level drinking to cope motives were positively associated with evening alcohol use $(b=.17, p=.09)$, and moderated the association between daily interpersonal conflict and evening drinking, although the effect was also marginal (Hypothesis $2_{\mathrm{d}}: b=-$ $.04, p=.09)$. Again, a negative association was revealed whereby the positive association between higher endorsement of coping motives (+1 standard deviation above the mean on coping motives) and evening alcohol use was only significant on days characterized by lower levels of interpersonal conflict at work (see Figure 4.7). 
Simple slopes revealed positive coping motive-daily drinking associations were significant at low levels of interpersonal conflict (-1 standard deviation; $b=.24, p=.05$ ), but were weaker on days participants experienced their typical (i.e., average; $b=.17$, $p=.09$ ) and high (+1 standard deviation; $b=.10, p=.28$ ) levels of interpersonal conflict. Hypothesis $3_{\mathrm{g}}$ predicting a three-way interaction between interpersonal conflict at work, coping motives, and average emotional support coping was not supported.

Moderating effects of coping motives and instrumental support coping. Similar effects were observed when examining instrumental support coping as a moderator, whereby no direct relationship between interpersonal conflict and daily drinking was observed $(b=-.02, p=.20)$, however higher endorsement of coping motives $(b=.21, p$ $=.03$ ) was associated with greater daily alcohol use. This association was qualified by a marginally significant cross-level interaction (Hypothesis $2_{\mathrm{d}}: b=-.04, p=.08$ ). Hypothesis $3_{\mathrm{h}}$ predicting a three-way interaction between interpersonal conflict at work, coping motives, and average instrumental support coping was not supported.

Role Overload - Likelihood of Drinking Associations. Using identical procedures to those utilized when examining levels of evening consumption, the next set of analyses examined whether daily experiences of job stress (role overload and interpersonal conflict) were associated with an increased likelihood of consuming alcohol that evening rather than increases in consumption per se. An initial level 1 was evaluated where evening drinking was regressed on experiences of role overload. Contrary to prediction, controlling for day of the week, level of alcohol consumed the 
previous day, and whether participants worked the following day, daily experiences of role overload were not significantly associated with likelihood of consuming alcohol later that evening (Hypothesis $1_{\mathrm{a}}: b=-.004, p=.88$ ). Results from all subsequent models are displayed in Table 4.2.

Moderating effects of coping motives and emotional support coping. Next, two models controlling for age and gender were tested to evaluate the moderating role of coping motives and emotional and instrumental support coping respectively.

Participants were no more likely to consume alcohol during the evening on days they experienced greater than average levels of role overload $(b=.02, p=.74)$. Person-level drinking to cope motives were not significantly associated with an increased likelihood of drinking that evening (Hypothesis $2_{\mathrm{a}}: b=.16, p=.29$ ), nor was the interaction between daily role overload and drinking to cope motives predicting likelihood of drinking. However, a marginally significant three-way, cross-level interaction was observed (Hypothesis $3_{\mathrm{a}}: b=-.19, p=.08$ ), although this effect failed to meet traditional statistical significance standards. As depicted in Figure 4.8, the predicted probability of drinking was lowest on days characterized by high levels of role overload among those endorsing stronger coping motives and who utilized higher emotional support coping. Those endorsing stronger coping motives and utilized lower emotional support coping had the highest likelihood of drinking irrespective of their level of daily role overload. Surprisingly, the predicted probability of drinking also increased slightly on high stress 
days among those endorsing low levels of coping motives but high emotional support coping strategies.

Moderating effects of coping motives and instrumental support coping. Once again, daily experiences of role overload were not associated with higher likelihood of drinking in models examining the moderating role of instrumental support coping $(b=$ $.03, p=.44$ ), nor did person-level coping motives (Hypothesis $2_{\mathrm{a}}: b=.20, p=.18$ ). No significant moderation effects were observed for these models (Hypothesis $3_{b}$ not confirmed).

Interpersonal Conflict at Work - Likelihood of Drinking. Similar to the previous models and contrary to prediction, controlling for day of the week, whether alcohol was consumed the previous day, and whether participants worked the following day, daily experiences of interpersonal conflict were not significantly associated with evening drinking (Hypothesis $1_{\mathrm{b}}: b=-.02, p=.69$ ).

Moderating effects of coping motives and emotional support coping. When testing the full model, daily experiences of interpersonal conflict at work $(b=-.05, p=$ $.23)$, person-level coping motives $(b=.13, p=.37)$, their interaction $(b=.03, p=.56)$, and the three-way interaction between interpersonal conflict at work, coping motives, and average emotional support coping $(b=.01, p=.98)$ were all nonsignificantly associated with likelihood of drinking (Hypothesis $3_{c}$ ).

Moderating effects of coping motives and instrumental support coping. Similar effects were observed when examining instrumental support coping as a moderator. 
The direct relationship between interpersonal conflict and daily drinking was marginal ( $b$ $=-.07, p=.08$ ). No significant cross-level interactions were observed (i.e., Hypotheses $2_{b}$ and $3 \mathrm{~d})$.

\section{Discussion}

Excessive drinking in the military remains pervasive despite efforts to reduce problematic alcohol use. In addition to steep individual level consequences and organizational concerns over combat readiness and fitness among those actively serving in the military (Bray et al., 2013; Stahre et al., 2009), such patterns represent substantial concerns for civilian employers, as heavy and problematic drinking among employees has been shown to contribute to deficits in performance (Fisher et al., 2000), accidents and occupational injury (Dawson, 1994), and missed work days (Frone, 2011). As more and more service members return to the civilian workforce, a more thorough conceptualization and understanding is needed of how and when drinking occurs, who might be vulnerable or susceptible to engaging in these behaviors, and factors which may mitigate more problematic patterns of use. The present study attempted to achieve this by examining daily patterns of alcohol use in response to occupational stress (role overload and interpersonal conflict) among employed veterans. Consistent with the Stressor Vulnerability Model (Cooper et al., 1992a), we further tested whether these patterns were more prominent among those endorsing motivations to drinking to cope, and whether more adaptive coping strategies could offset such motivations. 
Preliminary analyses uncovered the male and female service members did not differ in the average number of days they consumed alcohol over the course of the study, nor the average number of drinks they consumed on drinking days. This was somewhat surprising as the tendency for men to report greater quantity and frequency of alcohol use, alcohol misuse (e.g., binging), and problems associated with use are some of the most robust gender-based findings in the research literature (Cooper et al., 1992a; Kuntsche et al., 2006; Lehavot et al., 2014; Stahre et al., 2009; Wilsnack, Vogeltanz, Wilsnack, \& Harris, 2000). However, a large-scale epidemiological study by Jacobson and colleagues (2008) revealed that while male service members were more likely to report higher rates of binging and alcohol problems from baseline to follow-up, and were more likely to develop new patterns of binging and alcohol problems at follow-up, female service members were more likely than males to develop new onset heavy weekly drinking at follow-up. Consistent with theories of group membership and social influence (see Brown, 2000; Hogg, 2010; Sherif, 1936) it is plausible that female service members begin to adopt the behaviors and norms of the broader military culture following enlistment. As has been documented, a norm of heavy drinking exists within the military (Ames et al., 2007; Jones \& Fear, 2011; Pedersen, Marshall, Schell, \& Neighbors, 2015). Recent evidence among Army personnel participating in a phonebased substance use intervention suggested estimates of peer drinking exceeded actual levels of drinking, and that these perceptions predicted soldier's own drinking behavior (Neighbors et al., 2014). Consistent with this evidence, and in contrast with broader 
cultural norms and gender roles surrounding alcohol use in the US, over time, drinking levels of female service members may increase and more closely approximate those of male service members. This may have large consequences for the long-term health and readiness of female service members, in lieu of evidence suggesting women metabolize alcohol less efficiently and may become intoxicated at lower levels of drinking, and may be at heightened risk for certain health complications such as cirrhosis of the liver, hepatitis, and cancer as a function of excessive drinking (Mumenthaler, Taylor, O’Hara, \& Yesavage, 1999; Nolen-Hoeksema, 2004; Thomasson, 1995).

\section{Daily Role Overload}

In addition to its negative effect on job attitudes and satisfaction (Perrewé et al. 2005), evidence has implicated role overload with psychological strain, burnout, and work-family conflict (Harvey, Kelloway, \& Duncan-Leiper, 2003). However results from this study do not support that strain from daily perceptions of role overload also manifest in the form of increased alcohol use behavior. Participants in this study consumed significantly fewer drinks in the evening following days they experienced higher levels of role overload at work, although they were not significantly more or less likely to do so. It was hypothesized that this association would be moderated by endorsement of drinking to cope motives, yet despite evidence that endorsement of greater coping motives was associated with higher levels of daily alcohol use, this effect was significant only at low levels of daily role overload. That is, relative to those lower in coping motives, service members who endorsed higher coping motives drank more 
on days they experienced lower role overload. This is consistent with prior research suggesting those endorsing higher levels of coping motives (relative to lower) consume more alcohol in general (Cooper et al., in press; Kuntsche et al., 2005). On days they experienced higher than usual levels of role overload, however, both those lower and higher on coping motives reported similarly lower levels of drinking.

While this effect runs counter to our initial hypothesis, this may represent an adaptive response to daily job stress on behalf of service members. When the demands of work exceed one's real or perceived resources to meet those demands, it would be counterproductive to consume alcohol in response. Despite higher person-level motivations to do so, service members reduced their consumption on these high stress days. From an organizational standpoint, this is beneficial in that employees are reducing their level of alcohol use on days they have higher job demands suggesting they may be allocating additional resources toward the completion of job tasks rather than avoiding the stressful experience through alcohol consumption which may hinder job performance (Fisher et al., 2000).

A marginally significant three-way interaction further clarified this association, and revealed these effects to be a product of one's endorsement of both coping motives and use of emotional support coping strategies over the study period. Specifically, on days characterized by lower levels of role overload, those endorsing higher levels of drinking to cope motivations drank more during the evening relative to those lower in drinking to cope. This was attenuated among those who utilized more (but not less) 
emotional support coping. Only those individuals who endorsed higher levels of both coping motives and emotional support coping showed a pattern of consumption whereby alcohol use was lower on days they experienced higher levels of role overload, suggesting that use of these strategies mitigated the deleterious effect of coping motives on daily role overload-evening drinking associations. As such, the consistent use of strategies to regulate one's emotions among service members, particularly those who are motivated to drink when stressed, may protect against harmful forms of drinking in response to feeling overwhelmed and that they have too much to do at work.

A surprising finding in our study was that a positive within-person association between role overload and likelihood of drinking was observed for those endorsing lower levels of coping motives who utilized higher emotional support coping strategies over the course of the study. While this may seem counterintuitive, this effect is similar to one obtained by Park and colleagues (2004b) who found that while college students with higher coping motives drank less on higher stress days if they sought out support from others, the opposite effect was true for students lower in coping motives who sought support. In this view, more adaptive attempts at coping such as support seeking may be confounded with other motivations for drinking (e.g., social motives; Cooper et al., 1992b) and subsequent behavior among those lower in coping motives, whereas individuals higher in coping motives may prefer withdrawal and drinking in solitude during times of stress (cf. Armeli, Todd, \& Mohr, 2005). Prior research has suggested the desires or motives to socialize with others are stronger determinants of drinking in 
moderation (Cooper, 1994; Kuntsche et al., 2005), whereas motives to cope with stressors have been more strongly linked with alcohol problems (Cooper et al., 1995; Simons et al., 2005). More research is needed to disentangle what role other motives might play in influencing alcohol use among service members.

In contrast to findings surrounding the role of emotional support coping, the use of instrumental support coping strategies was shown to exacerbate motivated drinking patterns among employed service members in this sample. Findings revealed that those who endorse higher levels of coping motives and utilized more instrumental support coping strategies drank more on days they experienced higher than usual levels of role overload. Within the context of this specific stressor, this effect can be explained from a conservation of resources perspective (Hobfoll, 1989). Specifically, relative to gathering emotional support from others, instrumental support strategies such as seeking out advice from one's peers or coworkers on how to complete a task or solve a problem may be a more direct method of coping when one perceives they have too many demands at work. Such steps may directly involve coworkers or peers to help address and resolve situations wherein one feels overloaded. In theory, by relying on one's support resources and taking steps to resolve these experiences, one frees up resources to engage in other tasks or activities, which may include drinking.

Such findings highlight the importance of examining multiple forms of coping behavior as these may be associated with distinct patterns of motivated drinking. With respect to the drinking patterns of those motivated to cope with stress, turning toward 
close others in an attempt to alter one's emotional response to feeling overloaded may be more adaptive than seeking advice in order to take direct steps and action to reduce the stress itself. To the extent that participants in this study appraised daily experiences of role overload as being outside of their control, such evidence is consistent with the theoretical models of coping (Conway \& Terry, 1994; Cutrona, Shaffer, Wesner, \& Gardner, 2007; Park et al., 2004a) whereby efforts to change one's emotional response may be more successful when faced with stressors perceived to be beyond one's control, whereas controllable stressors may be managed more effectively by taking more direct action to alter the situation itself. An investigation of this possibility is beyond the scope of the current study.

\section{Daily Interpersonal Conflict}

Interpersonal conflicts with others have been characterized as some of the most stressful and negative experiences on can incur on a daily basis (Bolger et al., 1988). Forms of interpersonal conflict, including workplace incivility are thought to be wholly aversive, and may hinder or detract from one's personal growth and goal pursuit within an organization, leading to significant strain, including lower job satisfaction and engagement, burnout, and ill-health and health behavior (Crawford et al., 2010; LePine, Podsakoff, \& LePine, 2005; Melamed et al., 2006). However, results from the present study do not follow this trend. Specifically, participants reported drinking less alcohol on days characterized by higher levels of interpersonal conflict relative to low, but were not more or less likely to drink on these days. Contrary to expectation and theory, this 
negative association was strongest among those higher in coping motives, although their likelihood of consuming alcohol more generally was unchanged on high stress days. Furthermore, use of emotional and instrumental support coping strategies did not alter these associations. These effects are somewhat surprising in lieu of prior research and effects observed for role overload. Studies examining similar issues at the daily level have found that interpersonal conflict is associated with increases in alcohol use (Armeli et al., 2007; Mohr et al., 2001; 2005), and experimental evidence has suggested stressdrinking associations are strong for social stressors such as interpersonal conflict (Higgins \& Marlatt, 1979). However, these studies utilized broader definitions of interpersonal conflicts which encompassed negative interactions, criticisms, and conflicts across all life roles and relationships (e.g., peer, romantic partner, family, coworker). In contrast, our study focused explicitly on interpersonal conflicts that occurred at work. Although participants reported experiencing conflicts on nearly $40 \%$ of work days, these conflicts were appraised as being only slightly disruptive on average. It is possible that conflicts which arise at work, although common, may be less disruptive than those occurring in other life domains, and thus less apt to motivate alcohol use behavior in response.

\section{Alternative Explanations}

Nonsignificant findings and those which seem to contradict theory and past research are not uncommon when investigating motivated drinking patterns at the daily level (Grant, Stewart, \& Mohr, 2009; Hussong, Galloway, \& Feagans, 2005). Hussong and 
colleagues (2005) showed that, although college students endorsing higher coping motives reported stronger fear-daily drinking relationships, higher motives were associated with weaker (negative) sadness-daily drinking relationships, suggesting that individuals higher in coping motives drank less on days when they experienced higher levels of sad mood. Some have attempted to explain these and other discrepancies as being due in large part to differences in the level of analysis at which alcohol use and motives are being measured, as person-level hypotheses based on previous theory and evidence may not generalize to the daily-level (Kenny, Kashy, \& Bolger, 1998; Tennen et al., 2000). As such, counter-intuitive findings should be interpreted with caution.

An alternative explanation for the inconsistency of effects observed in this study is predicated on weekly patterns of drinking in this sample. Results indicated alcohol use was generally lower on weekdays (Sunday-Thursday) relative to weekend days (Friday-Saturday). Although the present investigation is underpowered to test such associations, it is possible that strain from role overload and interpersonal conflicts at work accumulate over the work week, and manifests in higher than average levels of drinking on the weekends rather than as a direct response to job stress following a work day. Indeed, a majority of work days occurred on Monday through Friday, and drinking levels were highest on Friday evenings. Given the nature of our sample (approximately $81 \%$ of our sample were married, $78 \%$ of which had children), insofar as job stress is demanding on both time and emotional resources, it may be that participants did not respond with greater drinking that day, rather increased their drinking at the end of the 
week. Future studies should examine alternative temporal intervals when investigating the complex within-person associations of job stressors on behavioral outcomes such as alcohol use.

\section{Implications for Intervention}

Reducing problematic alcohol use (e.g., binging, heavy drinking) is among the top priorities among military command (Bray et al., 2013). Therefore, providing appropriate strategies, resources, and skills to service members upon reentry to the civilian workplace is paramount. Despite the somewhat conflicting results obtained in our study, a wealth of evidence points to individual motivations for drinking as being critical points of intervention. Motivations and information about risks and consequences of use have been directly targeted as important supplements for existing alcohol-use interventions (Jacobson et al., 2008; Studer et al., 2014), with many advocating for treatment and prevention efforts to provide more tailored, specific feedback to individual experiences. In addition to pre-existing methods which promote effective coping among service members, motivational approaches which attend to individual beliefs, cognitions, and motivations surrounding alcohol use likely play a key role in determining the efficacy of such prevention and intervention efforts (Carey, ScottSheldon, Carey, \& DeMartini, 2007; Miller \& Rollnick, 1991, P.M. Miller et al., 2014; Wurdak, Wolstein, \& Kuntsche, 2016).

Brief educational interventions paired with individual feedback (e.g., McDevittMurphy et al., 2015b) provide alternative methods for coping which do not involve 
alcohol, therefore may reduce stress-related drinking and the development of later problems. Similar effects have been observed with college students, as coping skills trainings which incorporate psychosocial and cognitive risk factors have previously shown efficacy in the treatment of substance use and substance use disorders (e.g., Conrod, Stewart, Comeau, \& Maclean, 2006). Improved practices which promote social connection and encourage support seeking with an emphasis on relationship building would likely improve reintegration outcomes greatly. Supplementing these existing treatment modalities with a motivational component would provide service members with information and strategies more specific to their own needs and experience. Looking beyond the level of the individual, stress reduction in the workplace may also be achieved through interventions designed to improve organizational climate (Ames \& Bennett, 2011; Ames, Grube, \& Moore, 2000; Heaney, 2011; Lamontagne et al., 2007). By promoting change at multiple ecological levels (e.g., supervisor, work group, organizational), interventions may reduce interpersonal conflicts and incivility at work (Leiter, Day, Oore, \& Spence Laschinger, 2012), create more manageable workload and reduce potential for burnout and other forms of strain (Maslach, Leiter, \& Jackson, 2012).

\section{Strengths, Limitations and Future Directions}

One potential limitation stems from how drinking to cope motives were measured. Researchers have begun to explore episode-specific drinking motives as evidence has demonstrated significant cross-situational variability in drinking motives 
when predicting daily drinking behavior (Arbeau et al., 2011; O’Hara et al., 2014). However, doing so would have further limited the number of recorded observations, as the assessment of episode-specific motives is restricted to drinking days. Our evaluation of person level drinking to cope motives approximates a general tendency for participants to drink when stressed, and better reflects an individual-level vulnerability factor which is consistent with past theory (e.g., stressor-vulnerability model). When appropriate, future research should carefully consider the research question of interest prior to deciding whether assessing person-level or episode-specific motives are appropriate as they address different substantive questions.

Multilevel analyses revealed that within-person slopes for role overload and interpersonal conflict at work did not vary significantly from day to day which likely limited our ability to detect moderation effects. However, given our strong theoretical rationale for examining coping motives and support coping as vulnerability and protective factors for stress-related drinking respectively, examination of these variables as level-2 moderators was warranted (Bliese \& Jex, 2002).

Our use of single-item assessments for emotional and instrumental support coping represent a potential limitation and may increase measurement error in multilevel models. However, given the time-intensive nature and concerns over participant fatigue and frustration inherent in conducting daily process research, brief assessments which include single item constructs are often favored in a concerted effort to minimize participant burden rather than strict adherence to psychometric 
recommendations (Robins, Hendin, \& Trzesniewksi, 2001). This practice is common and has shown little loss of fidelity in research findings (Butler, Grzywacz, \& Bass, 2005; Park et al., 2004).

Additional concerns may exist about the representativeness of our sample relative to other studies of active duty personnel. In general, participants tended to be older, separated from the military, employed in a civilian role, married, and with 1 or more children. To the extent that younger, single service members with no children tend to drink more (Jacobson et al., 2008) and may be more apt to drink when stressed, our sample may be dissimilar from others investigating similar issues, and may explain some inconsistencies in our findings. However, $27 \%$ of the current sample met criteria for alcohol abuse based on a score of 8 or more on the Alcohol Use Disorders Identification Test (AUDIT) suggesting a sizeable minority of military service members continue to consume alcohol at high rates, some even more than a decade following separation. The extension of this line of inquiry to a community sample of employed service members provides a glimpse into the daily experiences of separated service members and reservists, and the role occupational stress, motivations, and coping behaviors play in influencing drinking patterns among this underrepresented group.

\section{Conclusion}

The present study provided what is, to our knowledge, the first daily process examination of alcohol use in response to occupational stress in a military sample. Results from this study depict a complex and inconsistent pattern of associations 
whereby some individuals were found to be more (or less) vulnerable to engaging in stress-related alcohol use depending on the type of stressor experienced, their level of endorsement of coping motives, and which coping strategies they typically use. In general, service members did not increase their drinking on days they experienced greater work stress, rather decreased their drinking on days of higher stress, even among those endorsing high levels of drinking to cope motivations. While this response to high demands and interpersonal conflicts may be functionally adaptive, this does not discount the high levels of drinking observed in this sample. Female service members in particular may be at risk for problematic patterns of drinking as their quantity and frequency of drinking did not differ from that of male service members over the course of the study. Military command and practitioners should be mindful of this and extend educational efforts to highlight risks of excessive drinking specific to women. As researchers continue to delve further into these topic areas, application of a more contextualized perspective toward tailored treatment efforts could yield benefits for the individuals and their families, organizations, and the military as a whole. 


\section{Footnotes}

${ }^{1}$ Participants in the daily study did not differ from non-participants on demographic characteristics (e.g., age, race, gender), risk for problem use assessed by the Alcohol Use Disorders Identification Test (AUDIT), or average alcohol use. However, as assessed at the baseline survey, daily study participants consumed alcohol on significantly more days in the past month relative to non-participants $\left(M_{\text {days }}=12.67\right.$ and 8.82 respectively; $\mathrm{t}(410)=-3.90, p<.001)$ 


\section{References}

Adler, D. A, Possemato, K., Mavandadi, S., Lerner, D., Chang, H., Klaus, J., ... Oslin, D. W. (2011). Psychiatric status and work performance of veterans of Operations Enduring Freedom and Iraqi Freedom. Psychiatric Services, 62(1), 39-46. doi:10.1176/appi.ps.62.1.39

Ames, G. M., \& Bennett, J. B. (2011). Prevention interventions of alcohol problems in the workplace: A review and guiding framework. Alcohol Research \& Health, 34, 175187.

Ames, G., \&Cunradi, C. (2004). Alcohol use and preventing alcohol-related problems among young adults in the military. Alcohol Research \& Health, 28, 252-257.

Ames, G. M., Cunradi, C. B., Moore, R. S., \& Stern, P. (2007). Military culture and drinking behavior among U.S. Navy careerists. Journal of Studies on Alcohol and Drugs, 68(3), 336-44. Retrieved from http://www.ncbi.nlm.nih.gov/pubmed/17446972

Ames, G. M., Grube, J. W., \& Moore, R. S. (2000). Social control and workplace drinking norms: a comparison of two organizational cultures. Journal of Studies on Alcohol, 61(2), 203-19. Retrieved from http://www.ncbi.nlm.nih.gov/pubmed/10757130

Armeli, S., Carney, M. A., Tennen, H., Affleck, G., \& O’Neil, T. (2000). Stress and alcohol use: A daily process examination of the stressor-vulnerability model. Journal of 
VULNERABILITY AND PROTECTIVE FACTORS OF STRESS-DRINKING

Personality and Social Psychology, 78(5), 979-994. doi:10.1037//0022-

3514.78.5.979

Armeli, S., Dehart, T., Tennen, H., Todd, M., \& Affleck, G. (2007). Daily Interpersonal Stress and the Stressor-Vulnerability Model of Alcohol Use. Journal of Social and Clinical Psychology, 26(8), 896-921. doi:10.1521/jscp.2007.26.8.896

Armeli, S., Todd, M., \& Mohr, C. D. (2005).A Daily Process Approach to Individual Differences in Stress-Related Alcohol Use. Journal of Personality, 73(6). doi:10.1111/j.1467-6494.2005.00362.x

Ball, S. A, Todd, M., Tennen, H., Armeli, S., Mohr, C. D., Affleck, G., \& Kranzler, H. R. (2007). Brief motivational enhancement and coping skills interventions for heavy drinking. Addictive Behaviors, 32(6), 1105-18. doi:10.1016/j.addbeh.2006.07.014

Bliese, P.D., \& Jex, S.M. (2002). Incorporating a multilevel perspective into occupational stress research: Theoretical, methodological, and practical implications. Journal of Occupational Health Psychology, 7, 265-276.

Blow, A. J., Gorman, L., Ganoczy, D., Kees, M., Kashy, D. a, Valenstein, M., ... Chermack, S. (2013).Hazardous drinking and family functioning in National Guard veterans and spouses post-deployment. Journal of Family Psychology, 27(2), 30313.doi:10.1037/a0031881

Bolger, N., Davis, A., \& Rafaeli, E. (2003). Diary methods: capturing life as it is lived. Annual Review of Psychology, 54, 579-616. doi:10.1146/annurev.psych.54.101601.145030 
Bolger, N., DeLongis, a, Kessler, R. C., \& Schilling, E. a. (1989).Effects of daily stress on negative mood. Journal of Personality and Social Psychology, 57(5), 808-18. Retrieved from http://www.ncbi.nlm.nih.gov/pubmed/2810026

Bray, R. M., Brown, J. M., \& Williams, J. (2013). Trends in binge and heavy drinking, alcohol-related problems, and combat exposure in the U.S. Military. Substance Use \& Misuse, 48, 799-810. doi:10.3109/10826084.2013.796990

Bray R. M., Marsden M. E., Peterson M. R. (1991). Standardized comparison of the use of alcohol, drugs, and cigarettes among military personnel and civilians. American Journal of Public Health, 81, 865-9.

Brown, R. (2000). Group processes: Dynamics within and between groups. (Chapter 2). Malden, MA: Blackwell Publishers.

Butler, A. B., Dodge, K. D., \& Faurote, E. J. (2010). College student employment and drinking: a daily study of work stressors, alcohol expectancies, and alcohol consumption. Journal of Occupational Health Psychology, 15(3), 291-303. doi:10.1037/a0019822

Butler, A. B., Grzywacz, J., Bass, B., \& Linney, K. (2005). Extending the demands-control model: A daily diary study of job characteristics, work-family conflict and workfamily facilitation. Journal of Occupational and Organizational Psychology, 78(2), 155-169. doi:10.1348/096317905X40097 
Carey, K. B., \&Correia, C. J. (1997). Drinking Motives Predict Alcohol-Related Problems in College Students * VAST of college students drink. Journal of Studies on Alcohol, $58,100-105$.

Carey, K. B., Scott-Sheldon, L. a J., Carey, M. P., \& DeMartini, K. S. (2007). Individual-level interventions to reduce college student drinking: A meta-analytic review. Addictive Behaviors, 32(11), 2469-2494. doi:10.1016/j.addbeh.2007.05.004

Carney, M. A, Armeli, S., Tennen, H., Affleck, G., \& O’Neil, T. P. (2000). Positive and negative daily events, perceived stress, and alcohol use: a diary study. Journal of Consulting and Clinical Psychology, 68(5), 788-798. doi:10.1037/0022006X.68.5.788

Carver, C. S. (1997). You want to measure coping but your protocol's too long: Consider the brief cope. International Journal of Behavioral Medicine, 4, 92-100.

Carver, C. S., \& Connor-Smith, J. (2010). Personality and Coping. Annual Review of Psychology, 61, 679-704.

Cohen, S., \& Wills, T. A. (1985). Stress, social support, and the buffering hypothesis. Psychological Bulletin, 98(2), 310-357.

Conger, J. J. (1956). Alcoholism: Theory, problem and challenge: II. Reinforcement theory and the dynamics of alcoholism. Quarterly Journal of Studies on Alcohol, 17, 296-305. 
Conrod, P. J., Stewart, S. H., Comeau, N., \& Maclean, M. (2006). Efficacy of cognitivebehavioral interventions targeting personality risk factors for youth alcohol misuse. Journal of Clinical Child and Adolescent Psychology, 35(4), 550-563. doi:10.1207/s15374424jccp3504

Conway,V. J., \& Terry, D. J. (1994).Appraised controllability as a moderator of the effectiveness of different coping strategies: A test of the goodness of fit hypothesis. Australian Journal of Psychology, 44, 1-7.

Cooper, M. L. (1994). Motivations for alcohol use among adolescents: Development and validation of a four-factor model. Psychological Assessment, 6, 117-128.

Cooper, M. L., Frone, M. R., Russell, M., \& Mudar, P. (1995). Drinking to regulate positive and negative emotions: A motivational model of alcohol use. Journal of Personality and Social Psychology, 69, 990-1005.

Cooper, M. L., Kuntsche, E., Levitt, A., Barber, L., \& Wolf, S. (in press). A motivational perspective on substance use: Review of theory and research. In K. J. Sher (Ed.), Oxford Handbook of Substance Use Disorders. New York, NY: Oxford University Press.

Cooper, M. L., Russell, M., \&Frone, M. R. (1990). Work stress and alcohol effects: a test of stress-induced drinking. Journal of Health and Social Behavior, 31(3), 260-276. doi:10.2307/2136891 
Cooper, M. L., Russell, M., Skinner, J. B., Frone, M. R., \&Mudar, P. (1992a). Stress and alcohol use: Moderating effects of gender, coping, and alcohol expectancies. Journal of Abnormal Psychology, 101, 139- 152.

Cooper, M. L., Russell, M. A. H., Skinner, J. B., \& Windle, M. (1992b). Development and validation of a three-dimensional measure of drinking motives. Psychological Assessment, 4(2), 123-132.

Cox, M., \& Klinger, E. (1988). A motivational model of alcohol use. Journal of Abnormal Psychology, 97, 168-180.

Crawford et al (2010). Linking job demands and resources to employee engagement and burnout: A theoretical extension and meta-analytic test. Journal of Applied Psychology, 95, 834-848.

Cucciare, M.A., Darrow, M., \& Weingardt, K.R. (2011). Characterizing binge drinking among U.S., military veterans receiving a brief alcohol intervention. Addictive Behaviors, 36, 362-367.

Cutrona, C.E., Shaffer, P.A., Wesner, K.A., \& Gardner, K.A. (2007). Optimally matching support and perceived spousal sensitivity. Journal of Family Psychology, 21.754758.

Dawson, D.A. (1994). Heavy drinking and the risk of occupational injury. Accident Analysis and Prevention, 26, 655-665.

DeLongis, A., \& Holtzman, S. (2005). Coping in context: The role of stress, social support, and personality in coping. Journal of Personality, 73, 2-24. 
Finlay, A. K., Ram, N., Maggs, J. L., \& Caldwell, L. L. (2012). Leisure activities, the social weekend, and alcohol use: evidence from a daily study of first-year college students. Journal of Studies on Alcohol and Drugs, 73(2), 250-9. Retrieved from http://www.ncbi.nlm.nih.gov/pubmed/22333332

Fisher, C. A., Hoffman, K. J., Austin-Lane, J., \& Kao, T. (2000). The relationship between heavy alcohol use and work productivity loss in active duty military personnel: A secondary analysis of the 1995 Department of Defense Worldwide Survey. Military Medicine, 5, 355-361.

Frone, M. R. (1999). Work Stress and Alcohol Use. Alcohol Research \& Health, 23, 284291.

Frone, M. R. (2008). Are work stressors related to employee substance use? The importance of temporal context assessments of alcohol and illicit drug use. Journal of Applied Psychology, 93(1), 199-206. doi:10.1037/0021-9010.93.1.199

Frone, M.R. (2011). Alcohol and illicit drug use in the workforce. In Quick, J. C. \& Tetrick, L. E. (Eds) Handbook of Occupational Health Psychology (2 ${ }^{\text {nd }}$ edition). Washington, DC: American Psychological Association.

Gaher, R. M., Simons, J. S., Hahn, A. M., Hofman, N. L., Hansen, J., \& Buchkoski, J. (2014). An Experience Sampling Study of PTSD and Alcohol-Related Problems. Psychology of Addictive Behaviors, 28, 1013-1025. 
Ganster, D. C., \& Rosen, C. C. (2013). Work Stress and Employee Health: A Multidisciplinary Review. Journal of Management. doi:10.1177/0149206313475815

Greeley, J., \& Oei, T. (1999). Alcohol and tension reduction. In K. E. Leonard \& H. T. Blane (Eds.), Psychological theories of drinking and alcoholism (2nd ed.) (pp. 14-53). New York, NY: Guilford Press.

Grunberg, L., Moore, S., Anderson-Connolly, R., \& Greenberg, E. (1999). Work stress and self-reported alcohol use: the moderating role of escapist reasons for drinking. Journal of Occupational Health Psychology, 4(1), 29-36. doi:10.1037/10768998.4.1.29

Harvey, S., Kelloway, E.K., \& Duncan-Leiper, L. (2003). Trust in management as a buffer of the relationships between overload and strain. Journal of Occupational Health Psychology, 8, 306-315.

Heaney, C. A. (2011). Worksite health interventions: Targets for change and strategies for attaining them. In J. C. Quick \& L. E. Tetrick (Eds.), Handbook of Occupational Health Psychology (2nd ed.). Washington, DC: American Psychological Association.

Heslin, K. C., Stein, J. A, Dobalian, A., Simon, B., Lanto, A. B., Yano, E. M., \& Rubenstein, L. V. (2012). Alcohol Problems as a Risk Factor for Postdisaster Depressed Mood Among U.S. Veterans. Psychology of Addictive Behaviors.doi:10.1037/a0030637 
Hobfoll, S. E. (1989). Conservation of resources: A new attempt at conceptualizing stress. American Psychologist, 44, 513-524. doi:10.1037/0003-066X.44.3.513

Hogg, M. A. (2010). Influence and leadership. In S. T. Fiske, D. T. Gilbert, \& G. Lindzey (Eds.), The handbook of social psychology (5th ed., pp. 1166 -1207).New York, NY: Wiley.

IOM (Institute of Medicine). (2012). Substance use disorders in the U.S. Armed Forces. Washington, DC: The National Academies Press.

Jacobson, I. G., Ryan, M. A. K., Hooper, T. I., Smith, T. C., Amoroso, P. J., Boyko, E. J., ... Bell, N. S. (2008). Alcohol Use and Alcohol-Related Problems Before and After Military Combat Deployment. JAMA, 300(6), 663-675.

Jones, E., \& Fear, N. T. (2011). Alcohol use and misuse within the military: a review. International Review of Psychiatry, 23(2), 166-72. doi:10.3109/09540261.2010.550868

Kuntsche, E., Knibbe, R., Gmel, G., \& Engels, R. (2005). Why do young people drink? A review of drinking motives. Clinical Psychology Review, 25(7), 841-861.

Kuntsche, E., Knibbe, R., Gmel, G., \& Engels, R. (2006). Who drinks and why? A review of socio-demographic, personality, and contextual issues behind the drinking motives in young people. Addictive Behaviors, 31(10), 1844-57.

Kuntsche, E., \& Kuntsche, S. (2009). Development and validation of the Drinking Motive Questionnaire Revised Short Form (DMQ-R SF). Journal of Clinical Child and Adolescent Psychology, 38(6), 899-908. doi:10.1080/15374410903258967 
Lamontagne, A. D., Keegel, T., Louie, A. M., Ostry, A., \& Landsbergis, P. A. (2005). A systematic review of the job-stress intervention evaluation literature, 1990 2005. Job Stress Evaluation, 13(3), 268-280.

Larimer, M. E., Palmer, R. S., \&Marlatt, G. A. (1999). Relapse prevention: An overview of Marlatt's cognitive behavioral model. Alcohol Research \& Health, 23, 151-160.

Lazarus, R. S., \& Folkman, S. (1984). Stress, appraisal, and coping. New York: Springer. Lehavot, K., Stappenbeck, C. A, Luterek, J. A, Kaysen, D., \& Simpson, T. L. (2014). Gender differences in relationships among PTSD severity, drinking motives, and alcohol use in a comorbid alcohol dependence and PTSD sample. Psychology of Addictive Behaviors, 28(1), 42-52. doi:10.1037/a0032266

Leiter, M. P., Day, A., Oore, D. G., \& Spence Laschinger, H. K. (2012). Getting better and staying better: assessing civility, incivility, distress, and job attitudes one year after a civility intervention. Journal of Occupational Health Psychology, 17(4), 425-34.doi:10.1037/a0029540

Lepine, J. A., Podsakoff, N. P., \& Lepine, M. A. (2005). A Meta-Analytic Test of the Challenge Stressor--Hindrance Stressor Framework: an Explanation for Inconsistent Relationships Among Stressors and Performance. Academy of Management Journal, 48(5), 764-775. doi:10.5465/AMJ.2005.18803921

Liu, S., Wang, M., Zhan, Y., \& Shi, J. (2009). Daily Work Stress and Alcohol Use: Testing the Cross-Level Moderation Effects of Neuroticism and Job Involvement. Personnel Psychology, 62(3), 575-597. doi:10.1111/j.1744-6570.2009.01149.x 
Luchman, J. N., \& González-Morales, M. G. (2013). Demands, control, and support: a meta-analytic review of work characteristics interrelationships. Journal of Occupational Health Psychology, 18(1), 37-52. doi:10.1037/a0030541

Maslach, C., Leiter, M. P., \& Jackson, S. E. (2012). Making a significant difference with burnout interventions : Researcher and practitioner collaboration. Journal of Organizational Behavior, 33, 296-300. doi:10.1002/job

McCreary, D. R., \& Sadava, S. W. (1998). Stress, drinking, and the adverse consequences of drinking in two samples of young adults. Psychology of Addictive Behaviors, $12,247-261$.

McDevitt-Murphy, M. E., Fields, J. A., Monahan, C. J., \& Bracken, K. L. (2015a). Drinking motives among heavy-drinking veterans with and without posttraumatic stress disorder. Addiction Research \& Theory, 23, 148-155.

McDevitt-Murphy, M. E., Williams, J. L., Murphy, J. G., Monahan, C. J., \& Bracken-Miller, K. L. (2015b). Brief intervention to reduce hazardous drinking and enhance coping among OEF/OIF/OND veterans. Professional Psychology: Research and practice, $46,83-89$.

Melamed, S., Shirom, A., Toker, S., Berliner, S., \& Shapira, I. (2006). Burnout and risk of cardiovascular disease: Evidence, possible causal paths, and promising research directions. Psychological Bulletin, 132, 327-353. 
Miller, P.M., Book, S., Thomas, S., Smith, J., Randall, P., \& Randall, C. (2014). The potential utility of drinking motive questions to screen at-risk drinking in socially anxious patients. Journal of Substance Use, 19, 225-228.

Miller, W. R., \& Rollnick, S. (1991). Motivational interviewing: Preparing people to change addictive behavior. New York: Guilford Press.

Milliken, C. S., Auchterlonie, J. L., \& Hoge, C. W. (2007). Longitudinal assessment of mental health problems among active and reserve component soldiers returning from the Iraq war. JAMA : The Journal of the American Medical Association, 298(18), 2141-2148.

Mohr, C. D., Armeli, S., Tennen, H., Carney, M. A., Affleck, G., \& Hromi, A. (2001). Daily interpersonal experiences, context, and alcohol consumption: crying in your beer and toasting good times. Journal of Personality and Social Psychology, 80(3), 489-500. Retrieved from http://www.ncbi.nlm.nih.gov/pubmed/11300581

Mohr, C. D., Armeli, S., Tennen, H., Temple, M., Todd, M., Clark, J., \& Carney, M. A. (2005). Moving beyond the keg party: a daily process study of college student drinking motivations. Psychology of Addictive Behaviors, 19(4), 392-403.

Mroczek, D.K., \& Almeida, D.M. (2004). The effect of daily stress, personality, and age on daily negative affect. Journal of Personality, 72, 355-378.

Mumenthaler, M.S., Taylor, J.L., O'Hara, R., Yesavage, J.A. (1999). Gender differences in moderate drinking effects. Alcohol Research \& Health, 23, 55-64. 
National Institutes for Alcohol Abuse and Alcoholism (2015). What is a standard drink?

Retrieved from: http://www.niaaa.nih.gov/alcohol-health/overview-alcoholconsumption/what-standard-drink.

Neighbors, C., Walker, D., Rodriguez, L., Walton, T., Mbilinyi, L., Kaysen, D., ... \&

Roffman, R. (2014). Normative misperceptions of alcohol use among substance abusing Army personnel. Military Behavioral Health, 2, 203-209.

Nezlek, J. B. (2001). Multilevel random coefficient analyses of event and interval contingent data in social and personality psychology research. Personality and Social Psychology Bulletin, 27, 771-785.

Nezlek, J.B. (2012). Multilevel modeling analyses of diary-style data. In M.R. Mehl and T.S. Conner (Eds.), Handbook of Research Methods for Studying Daily Life (pp. 357-383). The New York, NY: The Guilford Press.

Nolen-Hoeksema, S. (2004). Gender differences in risk factors and consequences for alcohol use and problems. Clinical Psychology Review, 24, 981-1010.

Norman, S. B., Schmied, E., \& Larson, G. E. (2014). Predictors of Continued Problem Drinking and Substance. Journal of Studies on Alcohol and Drugs, 75, 557-566.

Park, C. L., Armeli, S., \& Tennen, H. (2004a). Appraisal-coping goodness of fit: a daily internet study. Personality \& Social Psychology Bulletin, 30(5), 55869.doi:10.1177/0146167203262855 
Park, C. L., Armeli, S., \& Tennen, H. (2004b). The daily stress and coping process and alcohol use among college students. Journal of Studies on Alcohol, 65(January), $126-135$.

Pedersen, E.R., Marshall, G.N., Schell, T.L., \& Neighbors, C. (2015). Young adult Veteran perceptions of peers' drinking behavior and attitudes. Psychology of Addictive Behaviors. http://dx.doi.org/10.1037/adb0000120.

Peirce, R. S., Frone, M. R., Russell, M., \& Cooper, M. L. (1996). Financial stress, social support, and alcohol involvement: a longitudinal test of the buffering hypothesis in a general population survey. Health Psychology, 15(1), 38-47. Retrieved from http://www.ncbi.nlm.nih.gov/pubmed/8788539

Perrewé, P.L., Zellars, K.L., Rossi, A.M., Ferris, G.R., Kacmar, C.J., ...\& Hochwarter, W.A. (2005). Political skill: An antidote in the role overload-strain relationship. Journal of Occupational Health Psychology, 10, 239-250.

Raudenbush, S. W., \& Bryk, A. S. (2002). Hierarchical Linear Models (2nd ed.). Newbury Park, CA: Sage Publications.

Robins, R. W., Hendin, H. M., \& Trzesniewski, K. H. (2001). Measuring Global SelfEsteem: Construct Validation of a Single-Item Measure and the Rosenberg SelfEsteem Scale. Personality and Social Psychology Bulletin, 27(2), 151-161. doi:10.1177/0146167201272002

Sayette, M. A. (2000). Does drinking reduce stress? Alcohol Research \& Health, 23, 250255. 
Sherif, M. (1936). The psychology of social norms. New York: Harper.

Simons, J. S., Gaher, R. M., Correia, C. J., Hansen, C. L., \& Christopher, M. S. (2005). An affective-motivational model of marijuana and alcohol problems among college students. Psychology of Addictive Behaviors, 19(3), 326-34.

Simpson, T. L., Stappenbeck, C. A, Luterek, J. A, Lehavot, K., \& Kaysen, D. L. (2014). Drinking motives moderate daily relationships between PTSD symptoms and alcohol use. Journal of Abnormal Psychology, 123(1), 237- 247.

Sonnentag, S., \& Frese, M. (2013). Stress in Organizations. In W.C. Borman, D.R. Ilgen, \& R.J. Klimoski (Eds.) Comprehensive Handbook of Psychology, Volume Twelve: Industrial Organizational Psychology. New York: Wiley.

Stahre, M. A., Brewer, R. D., Fonseca, V. P., \& Naimi, T. S. (2009). Binge drinking among U.S. active-duty military personnel. American Journal of Preventive Medicine, 36, 208-217.

Steptoe, A., Wardle, J., Pollard, T. M., Canaan, L., \& Davies, G. J. (1996). Stress, social support and health-related behavior: A study of smoking, alcohol consumption and physical exercise. Journal of Psychosomatic Research, 41(2), 171-180.

Stone, A.A., Shiffman, S. (1994). Capturing momentary self-report data: A proposal for reporting guidelines. Annals of Behavioral Medicine, 24, 236-243. 
Studer, J., Baggio, S., Mohler-Kuo, M., Dermota, P., Daeppen, J.-B., \& Gmel, G. (2014). Differential association of drinking motives with alcohol use on weekdays and weekends. Psychology of Addictive Behaviors, 28(3), 651-658. doi:10.1037/a0035668

Swendsen, J. D., Tennen, H., Carney, M. A., Affleck, G., Willard, A., \& Hromi, A. (2000). Mood and Alcohol Consumption : An Experience Sampling Test of the SelfMedication Hypothesis. Journal of Abnormal Psychology, 109(2), 198-204. doi:10.1037//0021-843X.

Tabachnick, B.G., \& Fidell, L.S. (2007). Using Multivariate Statistics, $5^{\text {th }}$ ed. Boston: Pearson.

Tennen, H., Affleck, G., Armeli, S., \& Carney, M. A. (2000). A daily process approach to coping. Linking theory, research, and practice. The American Psychologist, 55(6), 626-636. doi:10.1037/0003-066X.55.6.626

Tennen, H., Affleck, G., Coyne, J. C., Larsen, R. J., \& Delongis, A. (2006). Paper and plastic in daily diary research: Comment on Green, Rafaeli, Bolger, Shrout, and Reis (2006). Psychological Methods, 11(1), 112-8. doi:10.1037/1082-989X.11.1.112

Thoits, P. A. (1995). Stress, coping and social support processes: Where are we? What next? Journal of Health and Social Behavior (Extra Issue), 53 - 79.

Thomasson, H.R. (1995) Gender differences in alcohol metabolism: physiological responses to ethanol. In M. Galanter (Ed.) Recent Developments in Alcoholism, Vol. 12. Alcoholism and Women, pp. 163-179. New York: Plenum. 
Wang, M., Liu, S., Zhan, Y., \& Shi, J. (2010). Daily work-family conflict and alcohol use: testing the cross-level moderation effects of peer drinking norms and social support. The Journal of Applied Psychology, 95(2), 377-86.doi:10.1037/a0018138

Wilsnack, R.W., Vogeltanz, N.D., Wilsnack, S.C., \& Harris, T.R. (2000). Gender differences in alcohol consumption and adverse drinking consequences: Cross-cultural patterns. Addiction, 95, 251-265.

Wurdak, M., Wolstein, J., \& Kuntsche, E. (2016). Effectiveness of a drinking-motivetailored emergency-room intervention among adolescents admitted to hospital due to acute alcohol intoxication - A randomized controlled trial. Preventive Medicine Reports, 3, 83-89.

Zohar, D. (1997). Predicting burnout with a hassle-based measure of role demands. Journal of Organizational Behavior, 18, 101-115. 
Table 4.0. Descriptive statistics and inter-correlations among study variables

\begin{tabular}{lcccccccc}
\hline & $\mathrm{M}$ & $\mathrm{SD}$ & 1 & 2 & 3 & 4 & 5 & 6 \\
\hline 1. Coping Motives & 1.66 & 0.92 & - & & & & & \\
2. Role Overload & 2.00 & 1.97 & $.240^{*}$ & - & & & & \\
3. Interpersonal Conflict & 1.30 & 1.42 & $.308^{* *}$ & $.488^{* *}$ & - & & & \\
4. Emotional Support Coping & 1.35 & 0.51 & .149 & $.301^{* *}$ & $.386^{* *}$ & - & & - \\
5. Instrumental Support Coping & 1.62 & 0.61 & .136 & $.372^{* *}$ & $.303^{* *}$ & $.678^{* *}$ & - & -.162 \\
6. Number of drinks consumed & 1.15 & 1.19 & $.212^{*}$ & .007 & .033 & -.135 & -.144 & $.531^{* *}$ \\
7. Drinking Days & 6.31 & 6.02 & .024 & -.124 & .028 & -.094 & -.144 \\
\hline Note. ${ }^{*} p<.05, * * p<.01$ & & & & & & & & \\
\hline
\end{tabular}


Table 4.1. Multilevel regression results for role overload, interpersonal conflict at work, emotional support coping, and alcohol use

\begin{tabular}{|c|c|c|c|c|}
\hline \multirow[b]{2}{*}{ Within-person effects } & \multicolumn{2}{|c|}{$\begin{array}{c}\text { Number of } \\
\text { Drinks }\end{array}$} & \multicolumn{2}{|c|}{ Drink Day } \\
\hline & $B$ & SE & $b$ & SE \\
\hline Role Overload & $-.050 * *$ & .019 & .016 & .047 \\
\hline Role Overload x Emotional Support Coping & -.046 & .036 & -.015 & .040 \\
\hline Role Overload x Coping Motives & $-.041 \dagger$ & .021 & -.010 & .035 \\
\hline Role Overload x Emotional Support Coping x Coping Motives & $-.130 \dagger$ & .074 & $-.186 \dagger$ & .106 \\
\hline \multicolumn{5}{|l|}{ Between-person effects } \\
\hline Emotional Support Coping & $-.531 * *$ & .168 & $-.460 *$ & .197 \\
\hline Coping Motives & $.176+$ & .098 & .158 & .149 \\
\hline Emotional Support Coping $x$ Coping Motives & $-.358+$ & .202 & $-.541+$ & .306 \\
\hline \multicolumn{5}{|l|}{ Within-person effects } \\
\hline Interpersonal Conflict & $-.033+$ & .019 & -.051 & .041 \\
\hline Interpersonal Conflict x Emotional Support Coping & $.051 \dagger$ & .027 & .055 & .062 \\
\hline Interpersonal Conflict $x$ Coping Motives & $-.042+$ & .025 & .029 & .049 \\
\hline Interpersonal Conflict $x$ Emotional Support Coping $x$ Coping Motives & .043 & .082 & .005 & .170 \\
\hline \multicolumn{5}{|l|}{ Between-person effects } \\
\hline Emotional Support Coping & $-.530 * *$ & .173 & $-.486 * *$ & .184 \\
\hline Coping Motives & $.170 \dagger$ & .098 & .133 & .149 \\
\hline Emotional Support Coping $x$ Coping Motives & $-.365 \dagger$ & .206 & $-.633+$ & .337 \\
\hline
\end{tabular}

Note. All models controlled for grand mean-centered age, gender, day-of-week effects, drinking which occurred the previous day, and whether the participant worked the next day;

$* * p<.01, * p<.05,+p<.10$ 
Table 4.2. Multilevel regression results for role overload, interpersonal conflict at work, instrumental support coping, and alcohol use

\begin{tabular}{|c|c|c|c|c|}
\hline \multirow[b]{2}{*}{ Within-person effects } & \multicolumn{2}{|c|}{$\begin{array}{l}\text { Number of } \\
\text { Drinks }\end{array}$} & \multicolumn{2}{|c|}{ Drink Day } \\
\hline & $B$ & SE & $b$ & $S E$ \\
\hline Role Overload & -.011 & .017 & .034 & .044 \\
\hline Role Overload x Instrumental Support Coping & .028 & .030 & $.083+$ & .046 \\
\hline Role Overload x Coping Motives & .001 & .015 & -.005 & .029 \\
\hline Role Overload x Instrumental Support Coping x Coping Motives & $.085^{*}$ & .036 & .061 & .052 \\
\hline \multicolumn{5}{|l|}{ Between-person effects } \\
\hline Instrumental Support Coping & $-.482 * *$ & .148 & $-.437^{*}$ & .204 \\
\hline Coping Motives & $.209 *$ & .092 & .203 & .151 \\
\hline Instrumental Support Coping x Coping Motives & -.141 & .149 & -.132 & .226 \\
\hline \multicolumn{5}{|l|}{ Within-person effects } \\
\hline Interpersonal Conflict & -.025 & .019 & $-.069+$ & .039 \\
\hline Interpersonal Conflict $x$ Instrumental Support Coping & .035 & .022 & .055 & .056 \\
\hline Interpersonal Conflict x Coping Motives & $-.044 \dagger$ & .025 & .015 & .052 \\
\hline Interpersonal Conflict $x$ Instrumental Support Coping $x$ Coping Motives & .029 & .046 & -.079 & .115 \\
\hline \multicolumn{5}{|l|}{ Between-person effects } \\
\hline Instrumental Support Coping & $-.471 * *$ & .151 & $-.427^{*}$ & .204 \\
\hline Coping Motives & $.210^{*}$ & .095 & .205 & .154 \\
\hline Instrumental Support Coping $x$ Coping Motives & -.130 & .162 & -.105 & .250 \\
\hline
\end{tabular}

Note. All models controlled for grand mean-centered age, gender, day-of-week effects, drinking which occurred the previous day, and whether the participant worked the next day;

$* * p<.01, * p<.05,+p<.10$ 


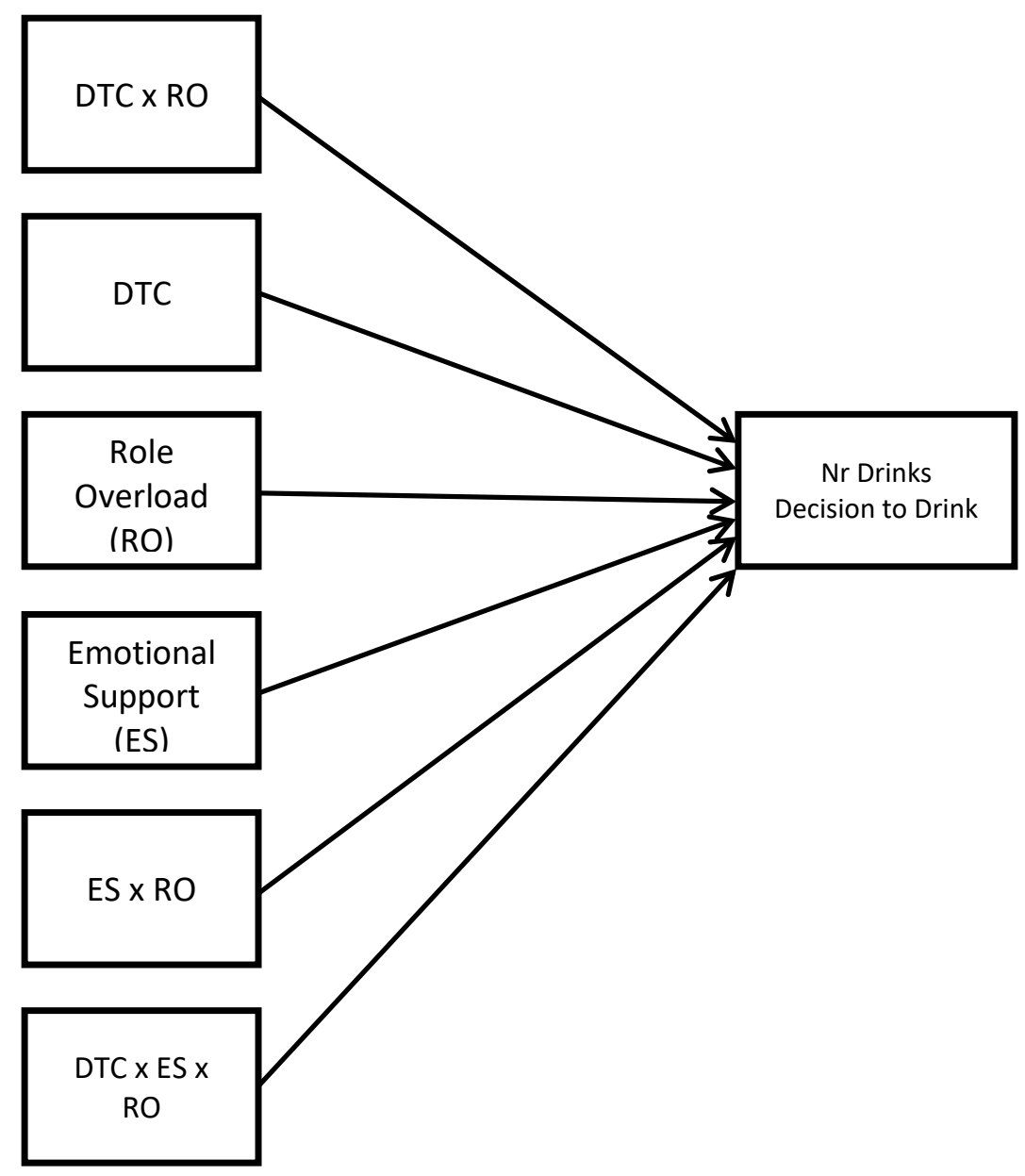

Figure 4.0. Hypothesized model of role overload (RO) predicting evening alcohol use and decision to drink as moderated by drinking to cope motives (DTC) and emotional support coping (ES) 


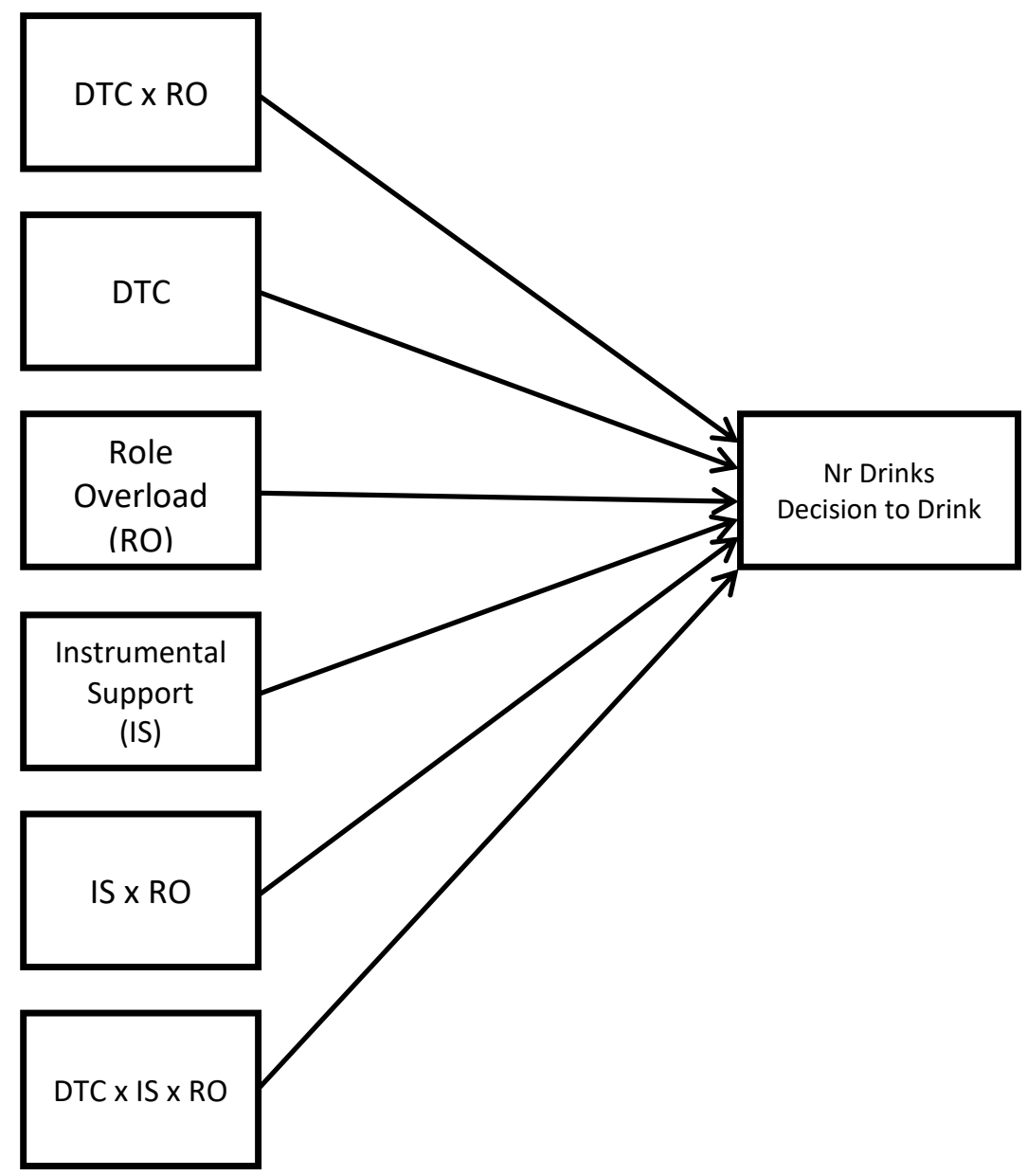

Figure 4.1. Hypothesized model of role overload (RO) predicting evening alcohol use and decision to drink as moderated by drinking to cope motives (DTC) and instrumental support coping (IS) 


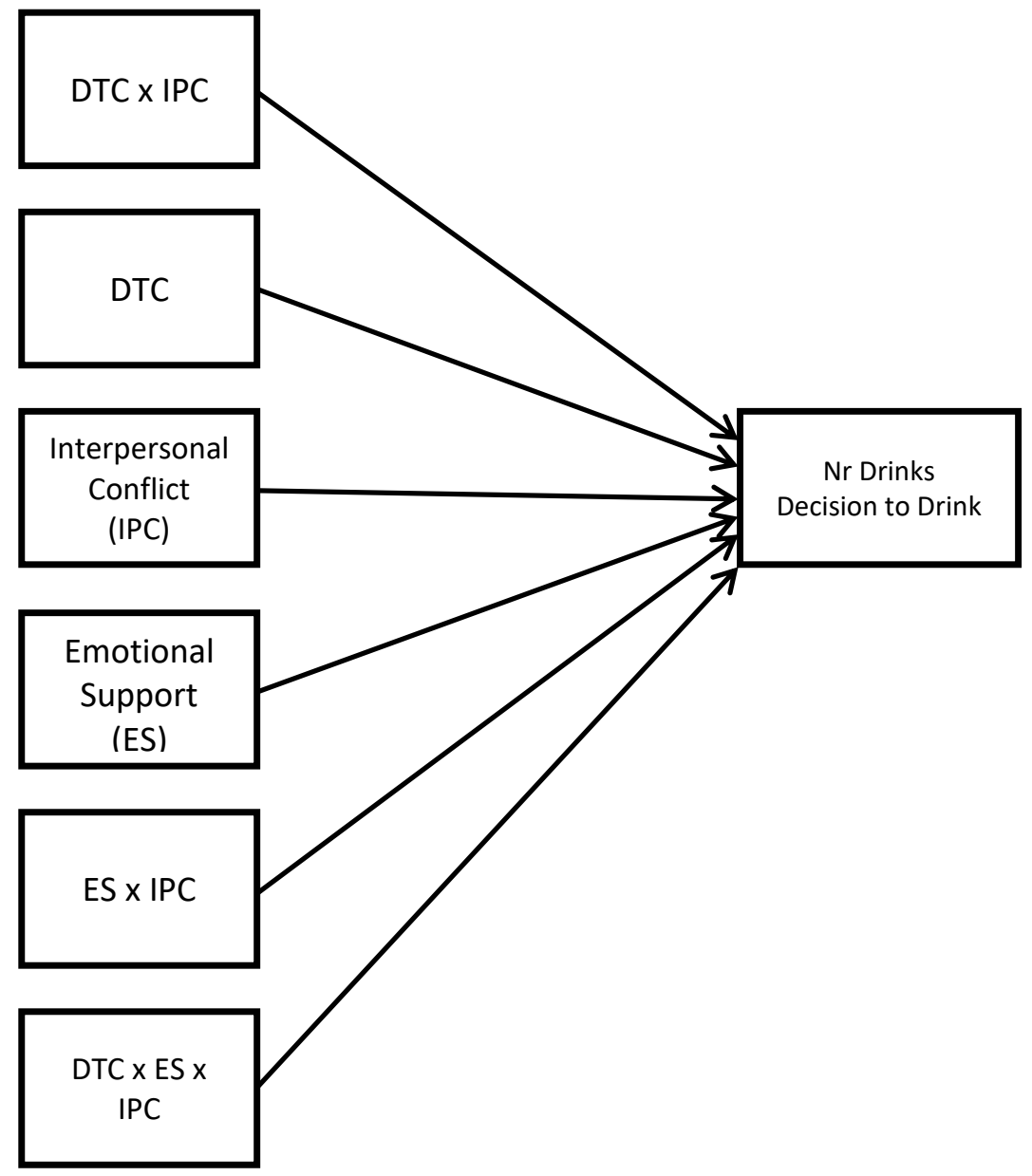

Figure 4.2. Hypothesized model of interpersonal conflict at work (IPC) predicting evening alcohol use and decision to drink as moderated by drinking to cope motives (DTC) and emotional support coping (ES) 


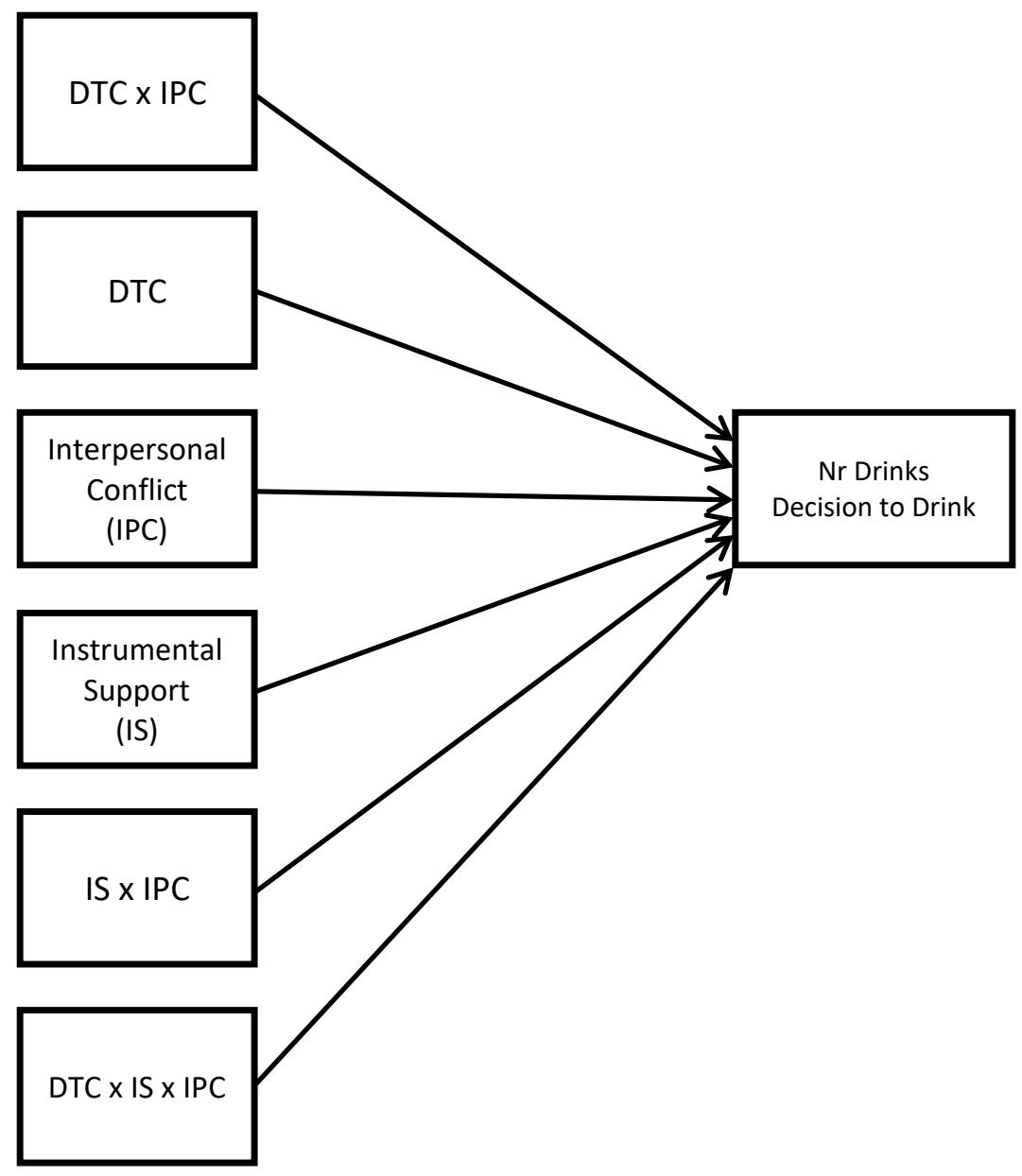

Figure 4.3. Hypothesized model of interpersonal conflict at work (IPC) predicting evening alcohol use and decision to drink as moderated by drinking to cope motives (DTC) and instrumental support coping (IS) 


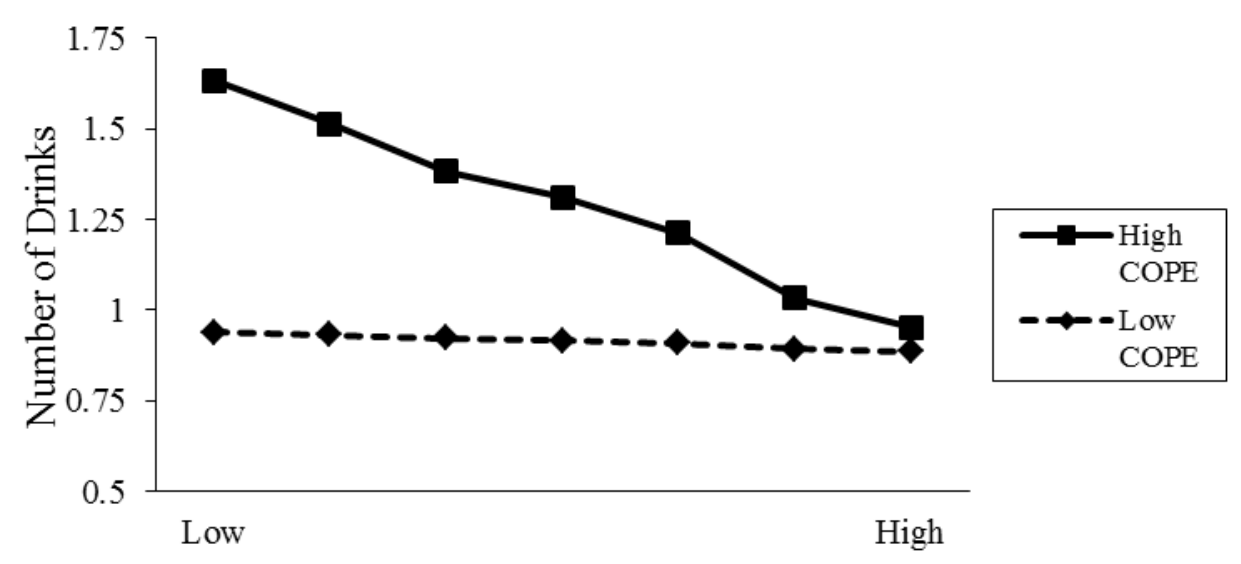

Role Overload

Figure 4.4. Marginally significant cross-level interaction between daily role overload and coping motives predicting level of evening consumption.

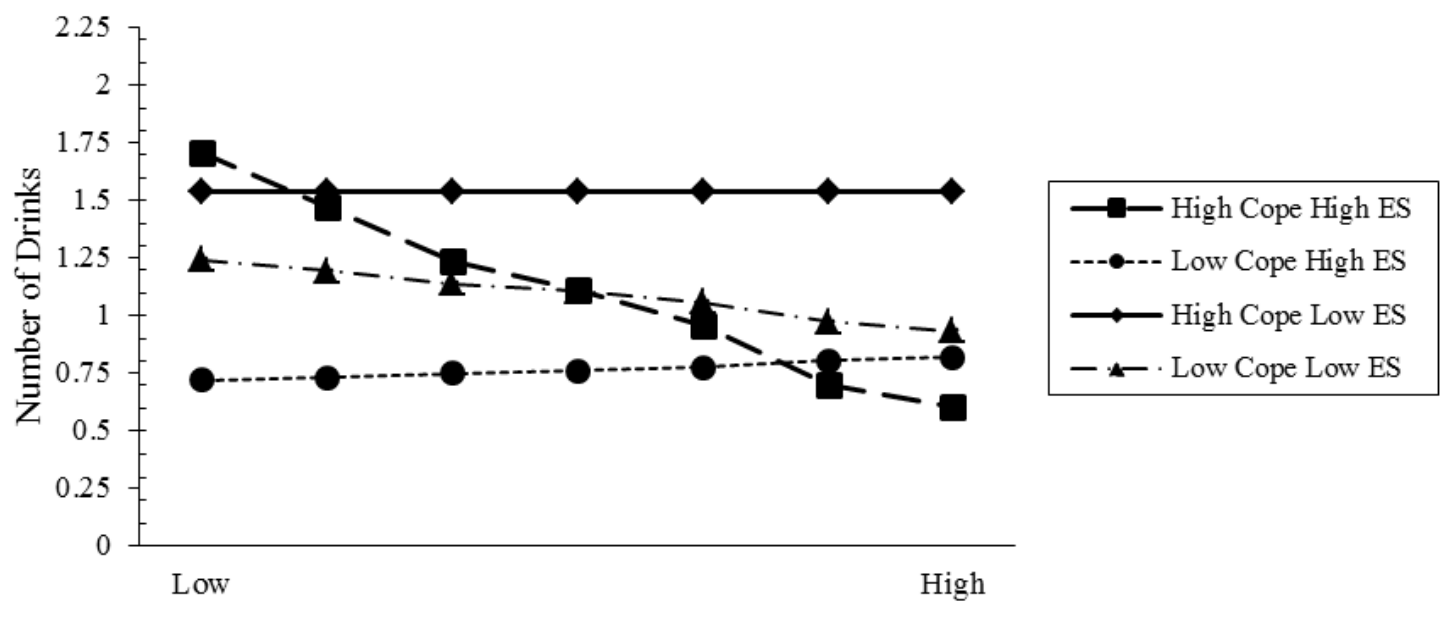

Role Overload

Figure 4.5. Marginally significant three-way cross-level interaction between daily role overload, coping motives, and average emotional support coping predicting level of evening consumption. 


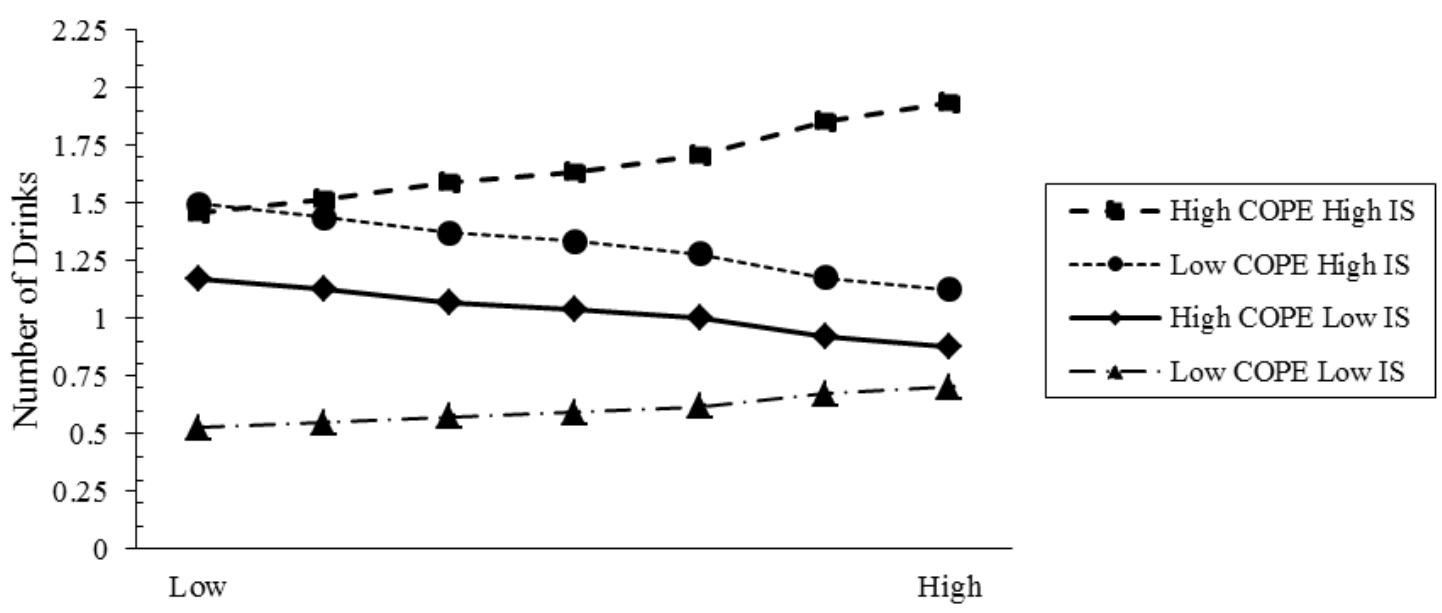

Role Overload

Figure 4.6. Three-way cross-level interaction between daily role overload, coping motives, and average instrumental support coping predicting level of evening consumption.

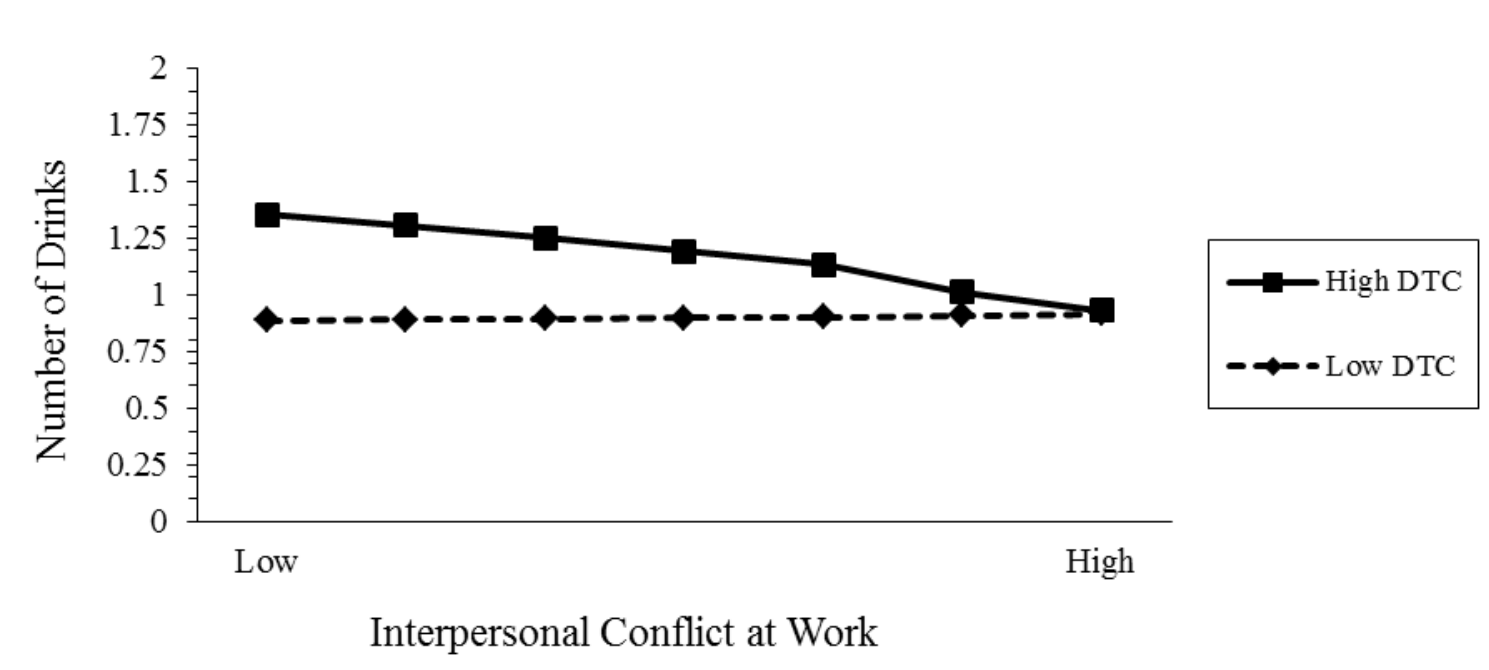

Figure 4.7. Marginally significant cross-level interaction between daily interpersonal conflict at work and coping motives predicting level of evening consumption. 


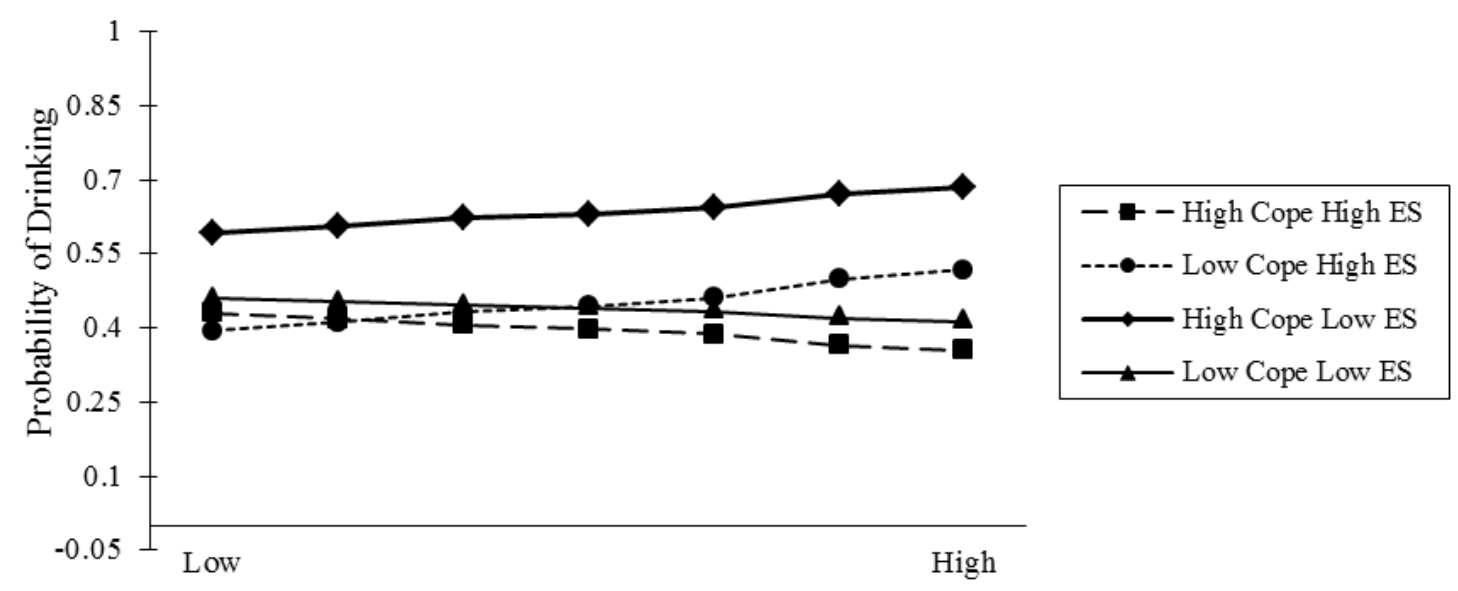

Role Overload

Figure 4.8. Marginally significant three-way cross-level interaction between daily role overload, coping motives, and average emotional support coping predicting probability of drinking that evening. 


\section{CHAPTER V. DISCUSSION}

Tension-reduction models of alcohol use (e.g., Conger, 1956) posit one of the primary reasons individuals consume alcohol is to suppress or avoid stress. It is paramount to understand these linkages given the unique association between drinking when stressed and alcohol problems (Cooper et al, 1995; Cooper, Russell, \& George, 1988; Simons et al., 2005). Yet researchers have long underscored that this direct association is insufficient for explaining the complex association between stress and alcohol involvement, with some individuals being more or less vulnerable for engaging in this behavior (Armeli, Carney, Tennen, Affleck, \& O’Neil, 2000; Cooper, Russell, Skinner, Frone, \& Mudar, 1992). Consistent with Stressor Vulnerability Models, this dissertation contributed to the body of work examining factors which precipitate or hinder stress-related drinking. Across three studies, I examined the interplay of how and when stress predicts drinking at multiple levels of analysis and with distinct populations shown to be at risk for engaging in high levels of alcohol use (i.e., college students and military service members). Results depict drinking to cope motives as an important mechanism through which stressors and mental health symptoms relate to drinking behavior and problems, whereas effective use of coping strategies and supportive resources may offset these associations. When determining susceptibility or vulnerability for problem alcohol use, these factors (i.e., alcohol use motivations and supportive relationships) represent two important areas warranting future research and intervention. 


\section{Strengths and Contributions}

The studies comprising this dissertation have several strengths and contribute to the ever-expanding literature on stress and alcohol involvement. First and foremost, they provide a thorough examination of vulnerability factors for a pattern of alcohol consumption shown to be linked with the development of alcohol problems and reduction in one's ability to cope successfully with perceived threats without the use of alcohol (Maisto, Carrey, \& Bradizza, 1999). In a 5-day study of college students, study 1 applied theoretical frameworks of stress and coping to explain links between a known vulnerability factor, extraversion (E), and daily alcohol use. Findings revealed that when stressed, higher E individuals were more likely to cope using direct, problem-focused strategies, which in turn were associated with lower levels of daily alcohol use. This suggests effective or appropriate coping strategies may disrupt a tendency to drink when stressed among these individuals. As an elaboration and extension of this work, studies 2 and 3 examined similar topics among another at risk population, military service members. Study 2 proposed that theoretical models of alcohol use motivation which exemplify the rationale underlying drinking behavior, can clarify why stress is linked with alcohol involvement. Results revealed that the association between posttraumatic stress symptoms (PTSS) and alcohol involvement among separated service members and reservists was indirect (i.e., mediated) through the endorsement of coping motivations. This finding indicates coping motives may be beneficial in explaining the observed link between posttraumatic stress symptoms and problematic 
alcohol use and involvement. Alternatively, Study 3 applied a motivational framework as a means of identifying who is more or less vulnerable for engaging in daily stress-related drinking. Although patterns of association were not always consistent with past theory and research, particularly on high stress days, those endorsing higher coping motives tended to drink more overall than those endorsing lower coping motives. This evidence is consistent with prior research suggesting a positive relationship between coping motives and alcohol involvement at the between- (e.g., Cooper et al., 1995; Cooper et al., in press; Kuntsche et al., 2005) and within-person levels of analysis (e.g., Grant, Stewart, \& Mohr, 2009; Mohr et al., 2005)

Of critical importance to the current studies is the identification of factors which buffer against harmful drinking behavior. Studies 2 and 3 explored protective factors in the form of perceptions of social support and use of emotional and instrumental support coping processes respectively. This approach distinguished the conditions under which stress is related to alcohol involvement. Study 2 examined the conditional nature of the aforementioned PTSS-alcohol involvement associations, and revealed the indirect association of PTSS on alcohol problems was shown to be conditional upon one's level of perceived support from friends. Specifically, perceiving that one is valued and supported by their peers was shown to buffer PTSS-coping motive associations. Interestingly, although support was hypothesized to protect against stress, in some circumstances it was shown to strengthen the association. For example, perceptions of support from family among service members in study 2 were shown to strengthen the 
association between coping motives and average level of alcohol use. Similarly, study 3 revealed mixed findings at the daily level whereby use of emotional support strategies buffered coping motive-alcohol consumption associations on high role overload days. In contrast, instrumental support strategies appeared to strengthen these associations on days participants experienced higher than typical demands at work. In this context, seeking advice on how to alter one's perspective and emotional response appeared more conducive to reducing drinking behavior whereas advice and support on how to resolve an issue was associated with increases in drinking. It is possible that support seeking in this manner involved drinking with friends and/or coworkers. Extraverted college students exhibited comparable patterns in study 1 , although this did not meet traditional significance standards. These findings add to others suggesting receipt (and likely provision) of social support and alcohol use behavior are often intertwined, and that support processes and drinking to cope should not be considered mutually exclusive activities (Aldridge-Gerry et al., 2011; Cohen \& LeMay, 2007; Park, Armeli \& Tennen, 2004).

More broadly, another key strength of this dissertation is its application theoretically-derived models of drinking motivations to explain alcohol use behaviors and consequences of military service members. With respect to our understanding of the role of alcohol use motivations in the stress-drinking process, studies 2 and 3 replicate and extend past research on drinking to cope motivations, and represent an important step in research on problem drinking among the military. The predictive 
power of alcohol use motivations more generally has been demonstrated in studies of alcohol involvement and drinking behavior among adolescents (Cooper, 1994), young adults and college students (Kuntsche et al., 2005; Mohr et al., 2005), communitydwelling adults (Crutzen \& Kuntsche, 2013), and also cross-culturally (Kuntsche et al., 2014), but have only recently been incorporated to the study of alcohol involvement in the military. A wealth of information has been gained through the identification of predictors, correlates, and consequences of alcohol use and misuse in the military (e.g., Bray et al., 2013, Crum-Cianflone et al., 2016; Hoge et al., 2004; Jacobson et al., 2008; Milliken, Auchterlonie, \& Hoge, 2007; Stahre et al., 2009), yet despite their obvious strengths, this work is not without its limitations. Much of the work informing current policy and intervention has been derived from examinations at the population level, and until recently has been largely atheoretical.

Study 2 provided additional evidence supporting alcohol use motivations as a final pathway through which stressful and emotional experiences relate to alcohol involvement. Previously, researchers have argued that mental health symptoms such as those accompanying PTSD and depression drive associations between trauma and combat exposure with alcohol involvement (see Kelley et al., 2013; Schumm \& Chard, 2012). The present work challenges this position and provided a rigorous test whereby the commonly observed association between PTSS and alcohol involvement was shown to be indirect through coping motives. Indeed when controlling for number of combat exposures and other well-documented correlates of alcohol involvement, coping 
motives emerged as a mediator of the association between PTSS and drinking quantity, frequency, problems, and binge drinking among service members. Results add to growing evidence of applicability of motivated perspectives to understanding alcohol involvement in the military (e.g., Lehavot, Stappenbeck, Luterek, Kaysen, \& Simpson, 2014; McDevitt-Muprhy et al., 2015a; Simpson et al., 2014; Whiteman \& Barry, 2011), and indicate theoretical models of motivated drinking are applicable to this high risk group as well.

Another strength of this dissertation is its novel design and unique sample of separated service members and active reservists employed in a civilian role. A criticism of the limited work exploring motivated drinking patterns in the military is that studies have relied heavily on clinical samples of treatment-seeking service members (Lehavot et al., 2014; Simpson et al., 2014) or those in primary care settings (McDevitt-Murphy et al., 2015a), and have almost exclusively examined the impact of more severe forms of mental health concerns such as PTSD, depression, and anxiety on alcohol involvement and development of alcohol use disorders (AUD). While Study 2 of this dissertation extended this line of research, study 3 remains the only study to our knowledge that examines the extent to which lower intensity, transient experiences of occupational stress influence subsequent drinking behavior among military service members. Furthermore, we know of no studies which have simultaneously explored factors which protect against these associations (i.e., perceptions of support and support coping). Evaluation of risk for development of alcohol misuse and abuse has been ubiquitous in 
the extant literature on alcohol involvement in the military. Drawing from prior research and theory, studies 2 and 3 assessed perceptions of support from multiple sources and alternative coping resources respectively (e.g., emotional and instrumental support coping) as potential buffers of this process. Collectively, the present set of studies provide an important glimpse into factors which may (a) exacerbate (e.g., drinking to cope motives) or (b) protect against problem alcohol use, and how these processes unfold among an underrepresented subset of military service members.

\section{What Have We Learned About Stress and Alcohol Use?}

Researchers and theorists have long claimed that direct associations between stress and alcohol use will remain weak when not accounting for personal, historical, situational, and cognitive influences surrounding use (Cox \& Klinger, 1988; Frone, 1999; Sadava \& Pak, 1993). In addition to examining multiple forms of stress (e.g., symptoms of posttraumatic stress from military service, role overload, interpersonal conflicts) with differing levels of severity, this dissertation contributed to this line of research, and addressed inconsistencies in stress-drinking associations through the analysis of (a) individual differences in vulnerability to stress-related drinking behavior, (b) distinct coping strategies, (c) alcohol use motivations, (d) support processes, and (e) by examining these associations at multiple levels of analysis. Yet evidence from the present studies reinforces the complex association that exists between stress and subsequent alcohol use in that stress was shown to predict increased drinking for some people (e.g., those endorsing higher coping motives) in some situations (e.g., on days 
college students used fewer problem-focused strategies, when perceptions of support from family are high among separated service members and reservists). Contextual factors surrounding the nature and composition of our sample, particularly for study 3 , may have influenced the associations observed in this dissertation. To the extent that this sample was comprised of service members balancing multiple life roles (e.g., employee, romantic partner, parent), competing demands from other roles may have precluded drinking on high stress days. In a similar vein, it may have been that desire to drink is most likely to increase in response to stress, but available resources (time, money, energy) may not be conducive to drinking behavior (Carney et al, 2000). Such findings further illustrate the limited utility of a direct stress-alcohol association, and corroborate recommendations from others to more thoroughly characterize the broader context surrounding alcohol use.

A related contribution of this work pertains to its examination of the association between stress and alcohol involvement at multiple levels of analysis. Methodologists have argued that patterns of association which emerge at the between-person level are not equivalent to those at the within-person level (Dvorak, Pearson, \& Day, 2014; Kenny, Kashy, \& Bolger, 1998; Stone et al., 1998; Tennen et al., 2000). When reflecting back upon a pre-determined timeframe (e.g., 30 days), participants may be relying on cognitive heuristics, and reflecting on one or a few salient events which may or may not accurately depict their experiences over the entire period (Armeli et al., 2007). Alternatively, a strength of daily process methods is that they reduce the likelihood of 
retrospective bias and increase the accuracy of reporting (Bolger, Davis, \& Raffaeli, 2003). Moreover, as argued previously, the basic tenets of both transactional models of coping (Lazarus \& Folkman, 1984) and motivational models of drinking are inherently process oriented, and are heavily influenced by factors related to the individual in a given situational context (Mohr et al., 2005; Tennen et al., 2000. Thus, the assessment of motivated drinking at the daily level may be the best approximation of the theoretical principles of these models (Dvorak et al., 2014). The decision to use such measures should ultimately be based on the nature and suitability of the method to answer a given research question should inform design decisions. A recent study by Gaher and colleagues (2014) astutely addressed this by stating that between-person analyses are best equipped to identifying individuals vulnerable to engaging in risky behavioral patterns, whereas within-person analyses are more appropriate for determining how (vulnerable) individuals adapt their behavior in response to environmental, situational, and emotional stimuli. To that effect, examination of the interplay of PTSS, person-level coping motives, and perceived support in study 2 was appropriate to explain PTSSalcohol involvement associations and who would be likely to exhibit those associations (i.e., those low in friend support). Examination of daily coping behaviors in studies 1 and 3 provided a more nuanced glimpse into specific behaviors potentially vulnerable participants engaged in on a daily level which altered stress-drinking associations. 


\section{Future Directions}

With respect to stress-related consumption among military service members, although a high proportion drink as a means of self-medicating stress (Cucciare et al., 2011; Schumm \& Chard, 2012), recent evidence suggests these efforts are not entirely successful (Simpson et al., 2014). What's more, excessive drinking may hinder recovery from more severe forms of mental health difficulties, including PTSD and depression (Schumm \& Chard, 2012). This dissertation has argued that theoretical models of drinking motivations are critical to understanding and identifying individual vulnerability for stress-related drinking. More importantly, these studies demonstrated the usefulness of this paradigm in describing how service members respond to both chronic (PTSS) and acute (role overload and interpersonal conflict) forms of stress. Studies examining the links between combat experiences, mental health, and alcohol have proposed a self-medication model whereby alcohol is used to avoid stress or temporarily suppress mental health symptoms. Models such as those presented by Schumm and Chard (2012) argue PTSD and associated symptoms represent the mediating mechanism between combat experiences and alcohol use disorders (see also Kelley et al., 2013). The present research extends this logic insofar as the inclusion of drinking to cope motives was identified as a potential mechanism through which PTSS contribute to all aspects of alcohol involvement (i.e., quantity and frequency of alcohol consumption, alcohol problems, and binge drinking). Further extrapolation of these selfmedication models would suggest multiple mediated pathways through which combat 
stressors are indirectly related to alcohol involvement and can negatively impact quality of life and well-being (Angkaw et al., 2015) of service members over time (see Figure 5.0). In contrast, supports from close others may mitigate associations between (a) combat trauma and PTSD, (b) PTSD and coping motives as found in study 2, and (c) coping motives and alcohol involvement. Such information could determine the longterm impact of drinking to cope on service member health, well-being, and quality of life.

Recommendations for military. Excessive alcohol use and consequences remain pervasive in the military. Each year, excessive drinking contributes to the early separation of an estimated 2,200 service members, and 10,400 service members who are unfit to deploy (Harwood et al., 2009). In June 2016, alcohol use was temporarily banned among all US personnel stationed in Japan following a series of alcohol-related incidents, one including an incident where two Japanese civilians were injured by a US service member driving under the influence of alcohol. Such events are costly to the military, both financially (e.g., legal and medical costs, lost work days) and to their reputation with allies, and are compounded when one considers the long-term disease burden of alcohol misuse. In addition to continued improvements to existing screening and treatment methods, a broader cultural shift in attitudes with respect to the acceptability of excessive alcohol use. Moreover, stigma associated with help seeking and other barriers to preventing, identifying, and treating problem alcohol use are critical to the continued success, fitness, and safety of service members (Santiago et al., 
2010). Policy changes in support of these efforts could include initiating less punitive measures for service members attempting to seek treatment in effort to reduce stigma and fear of reprisal. Military command and leaders also have a role to protect and promote safe and healthy behaviors (Bliese, 2006; Bliese, Adler, \& Castro, 2011), setting an example for the behaviors that are expected and condoned. Training and empowering military leadership in how to model safe behavior and identify those demonstrating problematic patterns of drinking, particularly drinking to cope with stress and mental health concerns could assist in effort to prevent and reduce hazardous drinking in the military.

Identification of coping motives as a strong mediator of PTSS-alcohol involvement associations further supports the utility of addressing the reasons why one is drinking in treatment efforts. When treating concomitant PTSD and problem alcohol use or disorders, the most efficacious method of treatment for clinicians is likely to address mental health symptomology in concert with alcohol use behavior (Hien et al., 2010; Schumm \& Chard, 2012). As drinking motives represent a "final pathway" (Cox \& Klinger, 1988) through which stress and mental health concerns influence alcohol involvement, pre-existing programs, including alcohol screening and brief educational interventions (e.g., McDevitt-Murphy et al., 2015b), may be bolstered by attending to individual beliefs, cognitions, and motivations surrounding alcohol use (Carey, ScottSheldon, Carey, \& DeMartini, 2007; Miller \& Rollnick, 1991, P.M. Miller et al., 2014; Wurdak, Wolstein, \& Kuntsche, 2016). Alcohol-related interventions have increased in 
locales such as the Veteran Health Administration (see Chavez et al., 2016), and incorporation of this perspective could assist service members in identifying potential "triggers" for their alcohol use behavior, thus providing a sense of agency and control, and reducing the likelihood of drinking as a form of stress or symptom management. Improved practices to promote healthy and effective alternative coping strategies among service members should promote social connection and encourage support seeking as a means of tempering reactivity to stress and mental health symptoms.

\section{Conclusion}

Results from three studies of two groups at risk for engaging in problematic alcohol use suggest drinking to cope motives are an important mechanism through which stressors and mental health symptoms relate to drinking behavior. Furthermore, whereas endorsement of such motives may increase one's likelihood of engaging in stress-related drinking, supportive relationships and reliance on social networks to cope with stress may offset these associations. When attempting to reduce problem drinking, research and practice should attend to the broader context surrounding drinking behavior, including the type of stress experienced and the motivations which underlie drinking behavior, and ways to effectively resolve stressful situations in ways that do not involve drinking. 


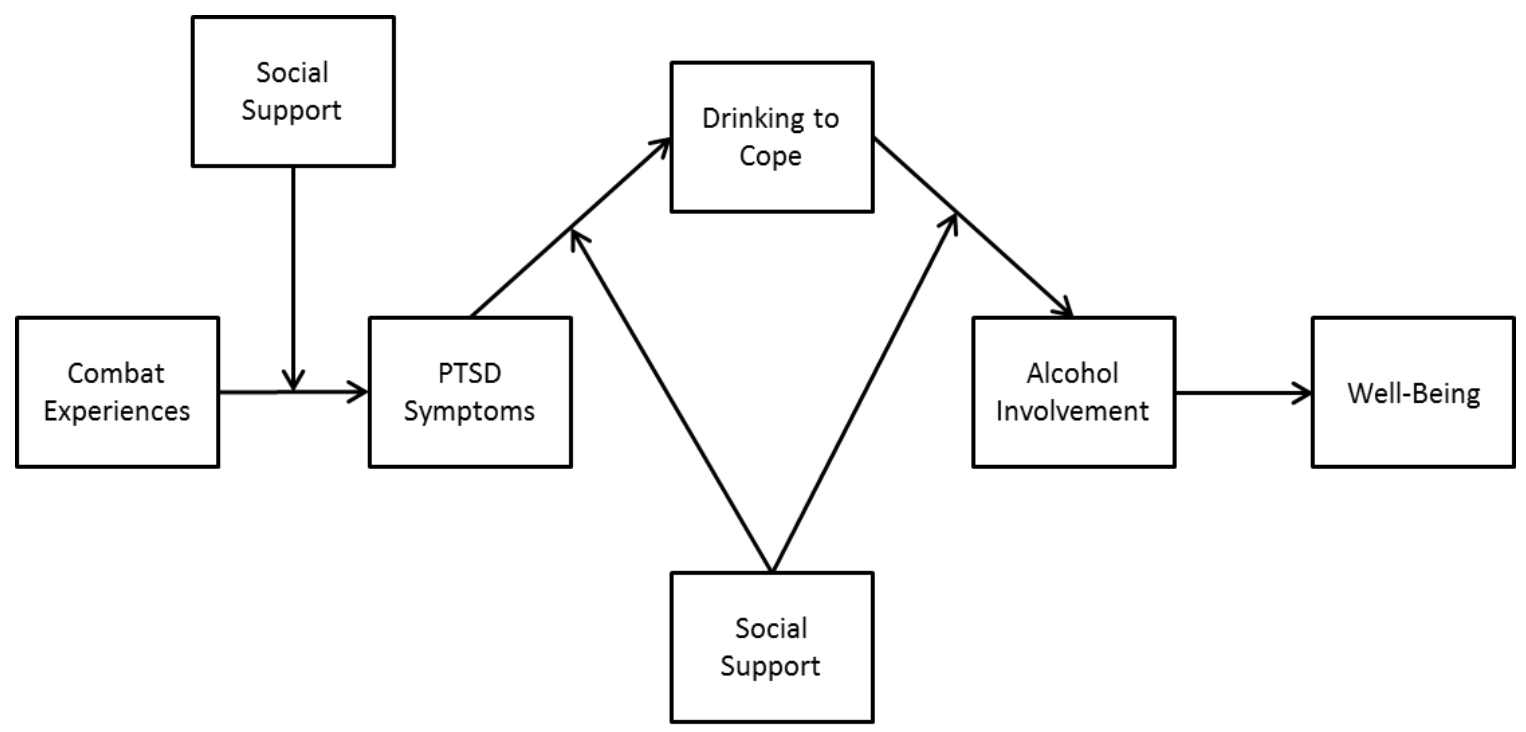

Figure 5.0. Theoretical model of self-medication process 
Terminal References

Adler, D. A., Possemato, K., Mavandadi, S., Lerner, D., Chang, H., Klaus, J., . . Oslin, D. W. (2011). Psychiatric status and work performance of veterans of Operations Enduring Freedom and Iraqi Freedom. Psychiatric Services, 62, 39-46. doi:

10.1176/appi.ps.62.1.39

Aldridge-Gerry, A. A., Roesch, S. C., Villodas, F., McCabe, C., Leung, Q., \& Da Costa, M. (2011). Daily stress and alcohol consumption: Modeling between-person and within-person ethnic variation in coping behavior. Journal of Studies on Alcohol and Drugs, 125-134.

Ames, G.M. and Cunradi, C.B. (2005). Alcohol use among young adults in the military: risk and prevention. Alcohol Research \& Health, 28(4), 252-257.

Amirkhan, J. H., Risinger, R. T., \& Swickert, R. J. (1995). Extraversion: A “hidden” personality factor in coping? Journal of Personality, 63, 189-212.

Angkaw, A.C., Haller, M., Pittman, J.O.E., Nunnink, S.E., Norman, S.B., .. \& Baker, D.G. (2015). Alcohol-related consequences mediating PTSD symptoms and mental health-related quality of life in OEF/OIF combat veterans. Military Medicine, 180, 670-674.

Armeli, S., Carney, M. a, Tennen, H., Affleck, G., \& O’Neil, T. P. (2000). Stress and alcohol use: a daily process examination of the stressor-vulnerability model. Journal of Personality and Social Psychology, 78(5), 979-994. doi:10.1037/0022- 
Armeli, S., Dehart, T., Tennen, H., Todd, M., \& Affleck, G. (2007). Daily Interpersonal Stress and the Stressor-Vulnerability Model of Alcohol Use. Journal of Social and Clinical Psychology, 26(8), 896-921.

Baer, J. S. (2002). Student factors: Understanding individual variation in college drinking. Journal of Studies on Alcohol, 14, 40-53.

Bliese, P.D. (2006). Social climates: Drivers of soldier well-being and resilience. In A.B. Adler, C.A. Castro, \& T.W. Britt (Eds.) Military life: The Psychology of Serving in Peace and Combat: Vol. 2. Operational Stress (pp. 213-234). Westport, CT: Praeger.

Bliese, P. D., Adler, A.B., \& Castro, C. A. (2011). Research-based preventive mental health care strategies in the military. In A.B. Adler, P.D. Bliese, \& C.A. Castro (Eds.) Deployment Psychology: Evidence-Based Strategies to Promote Mental Health in the Military (pp. 103-124). Washington, DC: American Psychological Association.

Blow, A. J., Gorman, L., Ganoczy, D., Kees, M., Kashy, D. a, Valenstein, M., ... Chermack, S. (2013). Hazardous drinking and family functioning in National Guard veterans and spouses postdeployment. Journal of Family Psychology, 27(2), 303-13. doi:10.1037/a0031881

Bolger, N., Davis, A., \& Rafaeli, E. (2003). Diary methods: capturing life as it is lived. Annual Review of Psychology, 54, 579-616. doi:10.1146/annurev.psych.54.101601.145030 
Bolger, N., \& Eckenrode, J. (1991). Social relationships, personality, and anxiety during a major stressful event. Journal of Personality \& Social Psychology, 61, 440-449.

Bolger, N., \& Zuckerman, A. (1995). A framework for studying personality in the stress process. Journal of Personality and Social Psychology, 69, 890-902.

Bouchery, E. E., Harwood, H. J., Sacks, J. J., Simon, C. J., \& Brewer, R. D. (2011). Economic costs of excessive alcohol consumption in the U.S., 2006. American Journal of Preventive Medicine, 41, 516-524.

Bray, R. M., Brown, J. M., \& Williams, J. (2013). Trends in binge and heavy drinking, alcohol-related problems, and combat exposure in the U.S. Military. Substance Use \& Misuse, 48, 799-810. doi:10.3109/10826084.2013.796990

Bray R. M., Marsden M. E., Peterson M. R. (1991). Standardized comparison of the use of alcohol, drugs, and cigarettes among military personnel and civilians. American Journal of Public Health, 81, 865-9.

Bray, R. M., Pemberton, M. R., Lane, M. E., Hourani, L. L., Mattiko, M. J., \& Babeu, L. a. (2010). Substance use and mental health trends among U.S. military active duty personnel: key findings from the 2008 DoD Health Behavior Survey. Military Medicine, 175(6), 390-399.

Bureau of Labor Statistics (2014). Charts from American Time Use Survey. Retrieved from: http://www.bls.gov/tus/charts/. 
Carey, K. B., \& Correia, C. J. (1997). Drinking Motives Predict Alcohol-Related Problems in College Students * VAST of college students drink. Journal of Studies on Alcohol, 58, 100-105.

Carey, K. B., Scott-Sheldon, L. a J., Carey, M. P., \& DeMartini, K. S. (2007). Individual-level interventions to reduce college student drinking: A meta-analytic review. Addictive Behaviors, 32(11), 2469-2494. doi:10.1016/j.addbeh.2007.05.004

Carney, M. A, Armeli, S., Tennen, H., Affleck, G., \& O’Neil, T. P. (2000). Positive and negative daily events, perceived stress, and alcohol use: a diary study. Journal of Consulting and Clinical Psychology, 68(5), 788-798. doi:10.1037/0022006X.68.5.788

Carrao, G., Bagnardi, V., Zambon, A., \& La Vecchia, C. (2004). A meta-analysis of alcohol consumption and the risk of 15 diseases. Preventive Medicine, 28, 613-619.

Catanzaro, S. J., \& Laurent, J. (2004). Perceived family support, negative mood regulation expectancies, coping, and adolescent alcohol use: Evidence of mediation and moderation effects. Addictive Behaviors, 29(9), 1779-1797. doi:10.1016/j.addbeh.2004.04.001

Chavez, L. J., Williams, E. C., Lapham, G. T., Rubinsky, A. D., Kivlahan, D. R., \& Bradley, K. a. (2016). Changes in patient-reported alcohol-related advice following Veterans Health Administration implementation of Brief Alcohol Interventions. Journal of Studies on Alcohol and Drugs, 77, 500-508. 
Cherpitel, C. J. (1993). Alcohol and injuries: A review of international emergency room studies. Addiction, 88, 923-937.

Clerkin, E. M., Werntz, A. J., Magee, J. C., Lindgren, K. P., \& Teachman, B. A. (2014). Evaluating Age Differences in Coping Motives as a Mediator of the Link Between Social Anxiety Symptoms and Alcohol Problems. Psychology of Addictive Behaviors, 28(3), 880-886.

Cohen, S., \& Lemay, E. P. (2007). Why would social networks be linked to affect and health practices? Health Psychology, 26(4), 410-417. doi:10.1037/02786133.26.4.410

Cohen, S., \& Wills, T. A. (1985). Stress, social support, and the buffering hypothesis. Psychological Bulletin, 98(2), 310-357.

Conger, J. J. (1956). Alcoholism: Theory, problem and challenge: II. Reinforcement theory and the dynamics of alcoholism. Quarterly Journal of Studies on Alcohol, $17,296-305$.

Cooper, M. L. (1994). Motivations for alcohol use among adolescents: Development and validation of a four-factor model. Psychological Assessment, 6, 117-128.

Cooper, M. L. (2002). Alcohol use and risky sexual behavior among college students and youth. Journal of Studies on Alcohol, 14 (Suppl.) 101-117.

Cooper, M. L., Agocha, V. B., \& Sheldon, M. S. (2000). A motivation perspective on risky behaviors: The role of personality and affect regulatory processes. Journal of Personality, 68, 1059-1088. 
Cooper, M. L., Frone, M. R., Russell, M., \& Mudar, P. (1995). Drinking to regulate positive and negative emotions: A motivational model of alcohol use. Journal of Personality and Social Psychology, 69, 990-1005.

Cooper, M. L., Kuntsche, E., Levitt, A., Barber, L., \& Wolf, S. (in press). A motivational perspective on substance use: Review of theory and research. In K. J. Sher (Ed.), Oxford Handbook of Substance Use Disorders. New York, NY: Oxford University Press.

Cooper, M. L., Russell, M., \& George, W. H. (1988). Coping, expectancies, and alcohol abuse: A test of social learning formulations. Journal of Abnormal Psychology, $97(2), 218-230$.

Cooper, M. L., Russell, M., Skinner, J. B., Frone, M. R., \& Mudar, P. (1992a). Stress and alcohol use: Moderating effects of gender, coping, and alcohol expectancies. Journal of Abnormal Psychology, 101, 139- 152.

Cooper, M. L., Russell, M. A. H., Skinner, J. B., \& Windle, M. (1992b). Development and validation of a three-dimensional measure of drinking motives. Psychological Assessment, 4(2), 123-132.

Corrao, G., Bagnardi, V., Zambon, A., \& La Vecchia, C. (2004). A meta-analysis of alcohol consumption and the risk of 15 diseases. Preventive Medicine, 38(5), 613-9.

Cox, M., \& Klinger, E. (1988). A motivational model of alcohol use. Journal of Abnormal Psychology, 97, 168-180. 
Coyne, J. C., \& DeLongis, A. (1986). Going beyond social support: the role of social relationships in adaptation. Journal of Consulting and Clinical Psychology, 54(4), 454-60. Retrieved from http://www.ncbi.nlm.nih.gov/pubmed/3745597

Crum-Cianflone, N.F., Powell, T.M., LeardMann, C.A., Russell, D.W., \& Boyko, E.J.(2016). Mental health and comorbidities in U.S. military members. Military Medicine, 181, 537-545.

Crutzen, R., \& Kuntsche, E. (2013). Validation of the four-dimensional structure of drinking motives among adults. European Addiction Research, 19(4), 222-226. doi:10.1159/000345457

Cucciare, M.A., Darrow, M., \& Weingardt, K.R. (2011). Characterizing binge drinking among U.S., military veterans receiving a brief alcohol intervention. Addictive Behaviors, 36, 362-367.

David, J. P., \& Suls, J. (1999). Coping efforts in daily life: Role of big five traits and problems appraisals. Journal of Personality, 67, 265-294.

DeLongis, A., \& Holtzman, S. (2005). Coping in context: The role of stress, social support, and personality in coping. Journal of Personality, 73, 2-24.

Demers, A. (2011). When Veterans Return: The Role of Community in Reintegration. Journal of Loss and Trauma, 16(February 2015), 160-179.

doi:10.1080/15325024.2010.519281 
Dvorak, R.D., Pearson, M.R., \& Day, A.M. (2014). Ecological momentary assessment of acute alcohol use disorder symptoms: Associations with mood, motives, and use on planned drinking days. Experimental and Clinical Psychopharmacology, 22, 285-297.

Feil, J., \& Hasking, P. (2008). The relationship between personality, coping strategies and alcohol use. Addiction Research and Theory, 16, 526-537.

Fisher, C. A., Hoffman, K. J., Austin-Lane, J., \& Kao, T. (2000). The relationship between heavy alcohol use and work productivity loss in active duty military personnel: A secondary analysis of the 1995 Department of Defense Worldwide Survey. Military Medicine, 5, 355-361.

Foran, H. M., Heyman, R. E., Slep, A. M. S., \& Snarr, J. D. (2012). Hazardous alcohol use and intimate partner violence in the military: Understanding protective factors. Psychology of Addictive Behaviors, 26(3), 471-83.

Fredrickson, B. L. (2001). The role of positive emotions in positive psychology. American Psychologist, 56(3), 218-226.

Frone, M. R. (1999). Work Stress and Alcohol Use. Alcohol Research \& Health, 23, 284291.

Gaher, R. M., Simons, J. S., Hahn, A. M., Hofman, N. L., Hansen, J., \& Buchkoski, J. (2014). An Experience Sampling Study of PTSD and Alcohol-Related Problems. Psychology of Addictive Behaviors, 28, 1013-1025. 
Ganster, D. C., \& Rosen, C. C. (2013). Work Stress and Employee Health: A

Multidisciplinary Review. Journal of Management. doi:10.1177/0149206313475815

Grant, V. V, Stewart, S. H., \& Mohr, C. D. (2009). Coping-anxiety and coping-depression motives predict different daily mood-drinking relationships. Psychology of Addictive Behaviors, 23(2), 226-37.

Greeley, J., \& Oei, T. (1999). Alcohol and tension reduction. In K. E. Leonard \& H. T. Blane (Eds.), Psychological theories of drinking and alcoholism (2nd ed.) (pp. 14-53). New York, NY: Guilford Press.

Halbesleben (2006). Sources of social support and burnout: A meta-analytic test of COR Model. Journal of Applied Psychology, 91, 1134-1145.

Harwood, H. J., Zhang, Y., Dall, T. M., Olaiya, R. S. T., \& Fagan, N. K. (2009). Economic implications of reduced binge drinking among the military health system's TRICARE Prime plan beneficiaries. Military Medicine, 174, 728-736.

Helgeson, V. S. (2003). Social support and quality of life. Quality of Life Research : An International Journal of Quality of Life Aspects of Treatment, Care and Rehabilitation, 12 Suppl 1, 25-31. Retrieved from http://www.ncbi.nlm.nih.gov/pubmed/12803308

Hien, D. A., Jiang, H., Campbell, A. N. C., Hu, M., Miele, G. M., Cohen, L., ... \& Nunes, E. V. (2010). Do treatment improvements in PTSD severity affect substance use outcomes? A secondary analysis from a randomized clinical trial in NIDA's Clinical Trials Network. American Journal of Psychiatry, 167, 95-101. 
Hoge, C. W., Castro, C. A., Messer, S. C., McGurk, D., Cotting, D. I., \& Koffman, R. L. (2004). Combat duty in Iraq and Afghanistan, mental health problems, and barriers to care. The New England Journal of Medicine, 351(1), 13-22.

Hull, J. G. (1981). A self-awareness model of the causes and effects of alcohol consumption. Journal of Abnormal Psychology, 90(6), 586-600.

Hussong, A. M., Galloway, C. A., \& Feagans, L. A. (2005). Coping Motives as a Moderator of Daily Mood-Drinking Covariation. Journal of Studies on Alcohol, (66), 344-353. IOM (Institute of Medicine). (2012). Substance use disorders in the U.S. Armed Forces. Washington, DC: The National Academies Press. Jacobson, I. G., Ryan, M. A. K., Hooper, T. I., Smith, T. C., Amoroso, P. J., Boyko, E. J., ... Bell, N. S. (2008). Alcohol Use and Alcohol-Related Problems Before and After Military Combat Deployment. JAMA, 300(6), 663-675.

Jones, E., \& Fear, N. T. (2011). Alcohol use and misuse within the military: a review. International Review of Psychiatry, 23(2), 166-72. doi:10.3109/09540261.2010.550868

Kaplan, M. S., Huguet, N., Feeny, D., McFarland, B. H., Caetano, R., Bernier, J., ... Ross, N. (2012). Alcohol use patterns and trajectories of health-related quality of life in middle-aged and older adults: a 14-year population-based study. Journal of Studies on Alcohol and Drugs, 73(4), 581-90. 
Kassel, J. D., Jackson, S. I., \& Unrod, M. (2000). Generalized Expectancies for Negative Mood Regulation and Problem Drinking among College Students * are motivated to engage. Journal of Studies on Alcohol, 61, 332-340.

Kehle, S. M., Ferrier-Auerbach, A. G., Meis, L. A., Arbisi, P. A., Erbes, C. R., \& Polusny, M. A. (2012). Predictors of postdeployment alcohol use disorders in National Guard soldiers deployed to Operation Iraqi Freedom. Psychology of Addictive Behaviors, 26(1), 42-50.

Kelley, M.L., Runnals, J., Pearson, M.R., Miller, M., Fairbank, J.A., ... \& Brancu, M. (2013). Alcohol use and trauma exposure among male and female veterans before, during, and after military service. Drug and Alcohol Dependence, 133, 615-624.

Kenny, D., Kashy, D., \& Bolger, N. (1998). Data analysis in social psychology. In D. Gilbert, S. Fiske, \& G. Linzey (Eds.), Handbook of Social Psychology (4 ${ }^{\text {th }}$ ed., pp. 233-265). New York: McGraw-Hill.

King, L. A, King, D. W., Fairbank, J. A, Keane, T. M., \& Adams, G. A. (1998). Resiliencerecovery factors in post-traumatic stress disorder among female and male Vietnam veterans: hardiness, postwar social support, and additional stressful life events. Journal of Personality and Social Psychology, 74(2), 420-34.

Kuntsche, E., Gabhainn, S. N., Roberts, C., Windlin, B., Vieno, A., Bendtsen, P., ... Wicki, M. (2014). Drinking motives and links to alcohol use in 13 European countries. Journal of Studies on Alcohol and Drugs, 75(3), 428-37. 
Kuntsche, E., Knibbe, R. A., Engels, R. C. M. E., \& Gmel, G. (2007). Bullying and fighting among adolescents - Do drinking motives and alcohol use matter? Addictive Behaviors, 32, 3131-3135.

Kuntsche, E., Knibbe, R., Gmel, G., \& Engels, R. (2005). Why do young people drink? A review of drinking motives. Clinical Psychology Review, 25(7), 841-861.

Kuntsche, E., Wiers, R. W., Janssen, T., \& Gmel, G. (2010). Same wording, distinct concepts? Testing differences between expectancies and motives in a mediation model of alcohol outcomes. Experimental and Clinical Psychopharmacology, 18(5), 436-44. doi:10.1037/a0019724

Kushner, M. G., \& Sher, K. J. (1993). Comorbidity of alcohol and anxiety disorders among college students: Effects of gender and family history of alcoholism. Addictive Behaviors, 18, 543-552.

Lazarus, R. S., \& Folkman, S. (1984). Stress, appraisal, and coping. New York: Springer. Lehavot, K., Stappenbeck, C. A, Luterek, J. A, Kaysen, D., \& Simpson, T. L. (2014). Gender differences in relationships among PTSD severity, drinking motives, and alcohol use in a comorbid alcohol dependence and PTSD sample. Psychology of Addictive Behaviors, 28(1), 42-52. doi:10.1037/a0032266

Levenson, R. W., Sher, K. J., Grossman, L. M., Newman, J., \& Newlin, D. B. (1980). Alcohol and Stress Response Dampening : Pharmacological Effects, Expectancy, and Tension Reduction. Journal of Abnormal Psychology, 89(4), 528-538. 
Levitt, A., \& Cooper, M. L. (2010). Daily alcohol use and romantic relationship functioning: evidence of bidirectional, gender-, and context-specific effects. Personality \& Social Psychology Bulletin, 36(12), 1706-22. doi:10.1177/0146167210388420

Maisto, S. A, Carey, K. B., \& Bradizza, C. M. (1999). Social Learning Theory. In K. E. Leonard \& H. T. Blane (Eds.), Psychological theories of drinking and alcoholism (2nd ed.) (pp. 106-163). New York, NY: Guilford Press.

McCabe, C. T., Roesch, S. C., \& Aldridge-Gerry, A. A. (2013). "Have a drink, you'll feel better." Predictors of daily alcohol consumption among extraverts: the mediational role of coping. Anxiety, Stress \& Coping, 26, 121-135.

McCrae, R. R. (Ed.). (1992). The five-factor model: Issues and applications [Special issue]. Journal of Personality, 60(2), 175-215.

McCreary, D. R., \& Sadava, S. W. (1998). Stress, drinking, and the adverse consequences of drinking in two samples of young adults. Psychology of Addictive Behaviors, $12,247-261$.

McDevitt-Murphy, M. E., Fields, J. A., Monahan, C. J., \& Bracken, K. L. (2015a). Drinking motives among heavy-drinking veterans with and without posttraumatic stress disorder. Addiction Research \& Theory, 23, 148-155. 
McDevitt-Murphy, M. E., Williams, J. L., Murphy, J. G., Monahan, C. J., \& Bracken-Miller, K. L. (2015b). Brief intervention to reduce hazardous drinking and enhance coping among OEF/OIF/OND veterans. Professional Psychology: Research and practice, $46,83-89$.

Miller, P.M., Book, S., Thomas, S., Smith, J., Randall, P., \& Randall, C. (2014). The potential utility of drinking motive questions to screen at-risk drinking in socially anxious patients. Journal of Substance Use, 19, 225-228.

Miller, W. R., \& Rollnick, S. (1991). Motivational interviewing: Preparing people to change addictive behavior. New York: Guilford Press.

Milliken, C. S., Auchterlonie, J. L., \& Hoge, C. W. (2007). Longitudinal assessment of mental health problems among active and reserve component soldiers returning from the Iraq war. JAMA : The Journal of the American Medical Association, 298(18), 2141-2148.

Mohr, C. D., Armeli, S., Tennen, H., Carney, M. A., Affleck, G., \& Hromi, A. (2001). Daily interpersonal experiences, context, and alcohol consumption: crying in your beer and toasting good times. Journal of Personality and Social Psychology, 80(3), 489-500.

Mohr, C. D., Armeli, S., Tennen, H., Temple, M., Todd, M., Clark, J., \& Carney, M. A. (2005). Moving beyond the keg party: a daily process study of college student drinking motivations. Psychology of Addictive Behaviors, 19(4), 392-403. 
Mohr, C. D., Averna, S., Kenny, D. a, \& Del Boca, F. K. (2001). “Getting by (or getting high) with a little help from my friends": an examination of adult alcoholics' friendships. Journal of Studies on Alcohol, 62, 637-645.

Mohr, C. D., Brannan, D., Wendt, S., Jacobs, L., Wright, R., \& Wang, M. (2013). Daily mood-drinking slopes as predictors: A new take on drinking motives and related outcomes. Psychology of Addictive Behaviors. doi:10.1037/a0032633

National Institute on Alcohol Abuse and Alcoholism (2000). Health risks and benefits of alcohol consumption. Alcohol Research \& Health, 24, 5-11.

National Institute on Alcohol Abuse and Alcoholism (2014). Alcohol Facts and Statistics. Retrieved from: http://www.niaaa.nih.gov/alcohol-health/overview-alcoholconsumption/alcohol-facts-and-statistics

Neighbors, C., Lee, C. M., Lewis, M. A., Fossos, N., \& Larimer, M. E. (2007). Are social norms the best predictor of outcomes among heavy- drinking college students? Journal of Studies on Alcohol and Drugs, 68, 556-565.

Nillni, Y. I., Gradus, J. L., Gutner, C. A., Luciano, M. T., Shipherd, J. C., \& Street, A. E. (2014). Deployment Stressors and Physical Health Among OEF / OIF Veterans : The Role of PTSD. Health Psychology, 33(11), 1281-1287.

O’Brien, T. B., \& DeLongis, A. (1996). The interactional context of problem-, emotion-, and relationship-focused coping: The role of the big five personality factors. Journal of Personality, 64, 775-813. 
Park, C. L., Armeli, S., \& Tennen, H. (2004). The daily stress and coping process and alcohol use among college students. Journal of Studies on Alcohol, 65, 126-135.

Patra, J., Taylor, B., Irving, H., Roerecke, M., Baliunas, D., \& Mohapatra, S. (2010).

Alcohol consumption and the risk of morbidity and mortality for different stroke types - a systematic review and meta-analysis. BMC Public Health, 10.

Paul, L. A., Grubaugh, A. L., Frueh, B. C., Ellis, C., \& Egede, L. E. (2011). Associations between binge and heavy drinking and health behaviors in a nationally representative sample. Addictive Behaviors, 36(12), 1240-5.

Pietrzak, R. H., Johnson, D. C., Goldstein, M. B., Malley, J. C., Rivers, A. J., Morgan, C. A., \& Southwick, S. M. (2010). Psychosocial buffers of traumatic stress, depressive symptoms, and psychosocial difficulties in veterans of Operations Enduring Freedom and Iraqi Freedom: The role of resilience, unit support, and postdeployment social support. Journal of Affective Disorders, 120(1-3), 188192. doi:10.1016/j.jad.2009.04.015

Rehm, J., Gmel, G., Sempos, C. T., \& Trevisan, M. (2002). Alcohol-Related Morbidity and Mortality. Alcohol Research \& Health, 27, 39-51.

Russell, D. W., Benedek, D. M., Naifeh, J. A., Fullerton, C. S., Benevides, N.,...\& Cacioppo, J. T. (2016). Social support and mental health outcomes among U.S. Army Special Operations personnel. Military Psychology. http://dx.doi.org/10.1037/mil000014 
Russell, D. W., Russell, C. A., Riviere, L. A, Thomas, J. L., Wilk, J. E., \& Bliese, P. D. (2014). Changes in alcohol use after traumatic experiences: The impact of combat on Army National Guardsmen. Drug and Alcohol Dependence, 139, 47-52. doi:10.1016/j.drugalcdep.2014.03.004

Sadava, S. W., \& Pak, A. W. (1993). Stress-related problem drinking and alcohol problems: A longitudinal study and extension of Marlatt's model. Canadian Journal of Behavioural Science, 25(3), 446-464. doi:10.1037/h0078841.

SAMHSA (2006). Results from the 2005 National Survey on Drug Use and Health: National Findings (DHHS Publication No. SMA 06-4194). Retrieved from http://www.oas.samhsa.gov/NSDUH/ 2k5NSDUH/ 2k5Results.htm

SAMHSA (2012). 2012 National Survey on Drug Use and Health (NSDUH). Table 6.90BHeavy Alcohol Use in the Past Month among Persons Aged 18 to 22, by College Enrollment Status and Demographic Characteristics: Percentages, 2011 and 2012. Available at: http://www.samhsa.gov/data/sites/default/files/NSDUHDetTabs2012/NSDUH-DetTabs2012/HTML/NSDUHDetTabsSect6peTabs55to107-2012.htm\#Tab6.90B

Santiago, P.N., Wilk, J.E., Milliken, C.S., Castro, C.A., Engel, C.C., \& Hoge, CW. (2010). Screening for alcohol misuse and alcohol-related behaviors among combat veterans. Psychiatric Services, 61, 575-581. 
Sayer, N. A., Noorbaloochi, S., Frazier, P., Carlson, K., Gravely, A., \& Murdoch, M. (2010). Reintegration Problems and Treatment Interests Among Iraq and Afghanistan Combat Veterans Receiving VA Medical Care. Psychiatric Services, 61(6), 589597.

Sayette, M. A. (2000). Does drinking reduce stress? Alcohol Research \& Health, 23, 250255.

Schumm, J.A., \& Chard, K.M. (2012). Alcohol and stress in the military. Alcohol Research: Current Reviews, 34, 401-407.

Sher, K. J., Bartholow, B. D., Peuser, K., Erickson, D. J., \& Wood, M. D. (2007). Stressresponse dampening effects of alcohol: Attention as a mediator and moderator. Journal of Abnormal Psychology, 116(2), 362-377.

Simons, J. S., Gaher, R. M., Correia, C. J., Hansen, C. L., \& Christopher, M. S. (2005). An affective-motivational model of marijuana and alcohol problems among college students. Psychology of Addictive Behaviors, 19(3), 326-34.

Simpson, T. L., Stappenbeck, C. A, Luterek, J. A, Lehavot, K., \& Kaysen, D. L. (2014). Drinking motives moderate daily relationships between PTSD symptoms and alcohol use. Journal of Abnormal Psychology, 123(1), 237-47.

Sonnentag, S., \& Frese, M. (2013). Stress in Organizations. In W.C. Borman, D.R. Ilgen, \& R.J. Klimoski (Eds.) Comprehensive Handbook of Psychology, Volume Twelve: Industrial Organizational Psychology. New York: Wiley. 
Stahre, M. A., Brewer, R. D., Fonseca, V. P., \& Naimi, T. S. (2009). Binge drinking among U.S. active-duty military personnel. American Journal of Preventive Medicine, 36, $208-217$.

Steptoe, A., Wardle, J., Pollard, T. M., Canaan, L., \& Davies, G. J. (1996). Stress, social support and health-related behavior: A study of smoking, alcohol consumption and physical exercise. Journal of Psychosomatic Research, 41(2), 171-180.

Stewart, S. H., \& Devine, H. (2000). Relations between personality and drinking motives in young adults. Personality and Individual Differences, 29, 495-511.

Stone, A. A., Schwartz, J. E., Neale, J. M., Shiffman, S., Marco, C. A., Hickcox, M., Paty, J., Porter, L. S., \& Cruise, L. J. (1998). A comparison of coping assessed by ecological momentary assessment and retrospective recall. Journal of Personality and Social Psychology, 74, 1670-1680.

Stone, A. A., \& Shiffman, S. (1994). Ecological momentary assessment (EMA) in behavioral medicine. Annals of Behavioral Medicine, 16, 199-202.

Taylor, S.E. (2010) Health. Handbook of Social Psychology. 2:II:19.

Tennen, H., Affleck, G., Armeli, S., \& Carney, M. a. (2000). A daily process approach to coping. Linking theory, research, and practice. The American Psychologist, 55(6), 626-636. doi:10.1037/0003-066X.55.6.626

Testa, M., \& Derrick, J. L. (2014). A daily process examination of the temporal association between alcohol use and verbal and physical aggression in community couples. Psychology of Addictive Behaviors, 28(1), 127-38. 
Thoits, P. A. (1995). Stress, coping and social support processes: Where are we? What next? Journal of Health and Social Behavior (Extra Issue), 53 - 79.

Thomas, J. L., Wilk, J. E., Riviere, L. A., Mcgurk, D., Castro, C. A., \& Hoge, C. W. (2010). Prevalence of Mental Health Problems and Functional Impairment Among Active Component and National Guard Soldiers 3 and 12 Months Following Combat in Iraq. Archives of General Psychiatry, 67(6), 614-623.

Wechsler, H., Dowdall, G.W., Maenner, G., Gledhill-Hoyt, J., \& Lee, H. (1998). Changes in binge drinking and related problems among American college students between 1993 and 1997: Results of the Harvard School of Public Health College Alcohol Study. Journal of American College Health, 47(2), 57-68.

Wechsler, H., \& Nelson, T. F. (2008). What We Have Learned from the Harvard School of Public Health College Alcohol Study: Focusing Attention on College Student Alcohol Consumption and the Environmental Conditions That Promote It. Journal of Studies on Alcohol and Drugs, 69, 481-490.

Whiteman, S.D., \& Barry A.E. (2011). A comparative analysis of student service member/veteran and civilian student drinking motives. Journal of Student Affairs Research and Practice, 48, 297-313.

Wilcox, S. (2010). Social relationships and PTSD symptomatology in combat veterans. Psychological Trauma: Theory, Research, Practice, and Policy, 2(3), 175-182. doi:10.1037/a0019062 
Wright, K. M., Foran, H. M., Wood, M. D., Eckford, R. D., \& McGurk, D. (2012). Alcohol Problems, Aggression, and Other Externalizing Behaviors After Return From Deployment: Understanding the Role of Combat Exposure, Internalizing Symptoms, and Social Environment. Journal of Clinical Psychology, 68(7), 782800.

Wurdak, M., Wolstein, J., \& Kuntsche, E. (2016). Effectiveness of a drinking-motivetailored emergency-room intervention among adolescents admitted to acute alcohol intoxication - A randomized controlled trial. Preventive Medicine Reports, 3, 83-89. 
PTSD Checklist - Military version (PCL-M) (Bliese, Wright, Adler, Cabrera, Castro, \& Hoge, 2008)

Below is a list of reactions that veterans sometimes have in response to stressful military experiences. Please read each one carefully and select how much you have been bothered by that problem in the PAST 30 DAYS.

$1=$ Not at all

$2=$ A little bit

$3=$ Moderately

4 = Quite a bit

$5=$ Extremely

1. Repeated disturbing memories, thoughts, or images of the stressful experience

2. Having physical reactions (like heart pounding, trouble breathing, sweating) when something reminded you of the stressful experience)

3. Avoiding activities or situations because they reminded you of the stressful experience

4. Having difficulty concentrating

\section{Alcohol use}

The following questions ask about your behavior over the PAST 30 DAYS...

1. On how many days did you consume alcohol?

2. How many alcoholic beverages did you have on a typical day in the past $\mathbf{3 0}$ days?

3. What was the largest number of alcoholic beverages that you drank in a single day?

4. Binge drinking (yes/no)

- DO IF (genderRx_v=0).

RECODE highalc_v (5 thru Highest=1) (Lowest thru 4=0) INTO Binge.

END IF.

VARIABLE LABELS Binge 'Binge drank (5+ men 4+ women)'.

EXECUTE.

SORT CASES BY Binge (A).

DO IF (genderRx_v=1).

RECODE highalc_v (4 thru Highest=1) (Lowest thru 3=0) INTO Binge.

END IF.

VARIABLE LABELS Binge 'Binge drank (5+ men 4+ women)'.

EXECUTE.

Drinking Motives Questionnaire - Revised - Short Form: Coping (DMQ-R-SF) (Kuntsche \& Kuntsche, 2009) 
Thinking of all the times you drink, how often do you drink for the following reasons?

$1=$ Never or almost never

$2=$ Some of the time

$3=$ Half of the time

$4=$ Most of the time

$5=$ Always or almost always

1. Because it helps you when you feel depressed or nervous

2. To cheer you up when you are in a bad mood

3. To forget about your problems

Alcohol Use Disorders Identification Test (AUDIT) (Saunders, Aasland, Babor, De La Fuente, \& Grant, 1993)

Thinking about your behavior over the PAST YEAR...

1. How often do you have a drink containing alcohol? [item removed from calculation]

$0=$ Never

$1=$ Monthly or less

$2=2$ to 4 a month

$3=2$ to 3 times a week

$4=4$ or more times a week

2. How many drinks containing alcohol do you have on a typical day when you are drinking? [item removed from calculation]

$0=1$ or 2

$1=3$ or 4

$2=5$ or 6

$3=7,8$, or 9

$4=10$ or more

3. How often do you have six or more drinks on one occasion? [item removed from calculation]

$0=$ Never

$1=$ Less than monthly

$2=$ Monthly

$3=$ Weekly

4 = Daily or almost daily

4. How often have you found that you were not able to stop drinking once you had started?

$0=$ Never

$1=$ Less than monthly

2 = Monthly

$3=$ Weekly 
4 = Daily or almost daily

5. How often have you failed to do what was normally expected from you because of drinking?

$0=$ Never

$1=$ Less than monthly

2 = Monthly

$3=$ Weekly

4 = Daily or almost daily

6. How often have you needed a first drink in the morning to get yourself going after a heavy drinking session?

$0=$ Never

$1=$ Less than monthly

2 = Monthly

$3=$ Weekly

4 = Daily or almost daily

7. How often have you had a feeling of guilt or remorse after drinking?

$0=$ Never

$1=$ Less than monthly

$2=$ Monthly

$3=$ Weekly

4 = Daily or almost daily

8. How often have you been unable to remember what happened the night before because you had been drinking?

$0=$ Never

$1=$ Less than monthly

2 = Monthly

$3=$ Weekly

4 = Daily or almost daily

9. Have you or someone else been injured as a result of your drinking?

$0=$ No

$2=$ Yes, but not in the last year

$4=$ Yes, during the last year

10. Has a relative or friend or a doctor or another health worker been concerned about your drinking or suggested you cut down?

$0=$ No

$2=$ Yes, but not in the last year

$4=$ Yes, during the last year 
Perceived Social Support Scale (PSS) (Procidano \& Heller, 1983)

The following statements refer to feelings and experiences which occur to most people at one time or another in their relationships with FRIENDS. Please read each statement carefully, and fill in the bubble which best represents your answer.

$1=$ Strongly disagree

$2=$ Disagree

$3=$ Neither agree nor disagree

$4=$ Agree

$5=$ Strongly agree

1. My friends give me the moral support I need

2. My friends enjoy hearing about what I think

3. I rely on my friends for emotional support

4. There is a friend I could go to if I were just feeling down, without feeling funny about it later

5. My friends are sensitive to my personal needs

6. My friends are good at helping me solve problems

7. I've recently gotten a good idea about how to do something from a friend

The following statements refer to feelings and experiences which occur to most people at one time or another in their relationships with FAMILY. Please read each statement carefully, and fill in the bubble which best represents your answer

$1=$ Strongly disagree

$2=$ Disagree

$3=$ Neither agree nor disagree

$4=$ Agree

$5=$ Strongly agree

1. My family give me the moral support I need

2. My family enjoy hearing about what I think

3. I rely on my family for emotional support

4. There is a family I could go to if I were just feeling down, without feeling funny about it later

5. My family are sensitive to my personal needs

6. My family are good at helping me solve problems

7. I've recently gotten a good idea about how to do something from a family

\section{Demographics}

1. What is your age? (Years) 
2. What is your gender

$$
\begin{aligned}
& 0=\text { Male } \\
& 1=\text { Female }
\end{aligned}
$$

Neuroticism (N) (Donnellan, Oswald, Baird, \& Lucas, 2006)

The following are statements generally used to describe people's behaviors. Please use the rating scale below to indicate how accurately each statement describes YOU.

$1=$ Very accurate

2 = Moderately accurate

$3=$ Neither inaccurate or accurate

4 = Moderately inaccurate

$5=$ Very inaccurate

1. I have frequent mood swings

2. I am relaxed most of the time

3. I get upset easily

4. I seldom feel blue

Combat Exposure Scale (CES) (Hoge et al., 2004)

How often did you experience the following during your MOST RECENT DEPLOYMENT?

$$
\begin{aligned}
& 1=\text { Never } \\
& 2=\text { One time } \\
& 3=2 \text { to } 4 \text { times } \\
& 4=5 \text { or more times }
\end{aligned}
$$

1. Being attacked or ambushed

2. Receiving small arms fire

3. Seeing dead bodies or human remains

4. Seeing dead or seriously injured Americans

5. Knowing someone seriously injured or killed

6. Improvised explosive device (IED)/booby trap exploded near you

7. Being physically moved or knocked over from an explosion

8. Being in threatening situations where you were unable to respond because of rules of engagement

9. Shooting or directing fire at the enemy

10. Engaging in hand-to-hand combat

11. Clearing/searching homes or buildings

12. Witnessing brutality/mistreatment toward non-combatants

13. Being wounded/injured

14. Seeing ill/injured women or children who you were unable to help

15. Receiving incoming artillery, rocket, or mortar fire 
16. Being directly responsible for the death of an enemy combatant

17. Feeling directly responsible for the death of a non-combatant

18. Feeling responsible for the death of US or ally personnel

19. Having a member of your own unit become a casualty

20. Had a close call, was shot or hit but protective gear saved you

21. Had a buddy shot or hit who was near you

22. Had a close buddy seriously injured or killed

23. Participating in IED/mine clearing operations

24. Saved the life of a Soldier or civilian

25. Observing abuse of Laws of War/Geneva Convention (e.g., weapons cached in Mosques, schools, or hospitals)

26. Encountering sniper fire

27. Believed you would be seriously injured or killed 
APPENDIX B: Study 3 Measures

Drinking Motives Questionnaire - Revised - Short Form: Coping (DMQ-R-SF) (Kuntsche \& Kuntsche, 2009)

Thinking of all the times you drink, how often do you drink for the following reasons?

$1=$ Never or almost never

2 = Some of the time

$3=$ Half of the time

$4=$ Most of the time

5 = Always or almost always

1. Because it helps you when you feel depressed or nervous

2. To cheer you up when you are in a bad mood

3. To forget about your problems

Role Hassles Index: Role overload (Zohar, 1997)

How emotionally or physically disruptive were the following events TODAY?

$0-\mathrm{N} / \mathrm{A}$

1 - Not Disruptive

2 - Slightly Disruptive

3 - Quite Disruptive

4 - Very Disruptive

1. Felt under time pressure, had a difficulty due to insufficient time

2. Had too much work - too many things to take care of

3. Had to stay too many extra hours or do inconvenient shift-work schedules

Role Hassles Index: Interpersonal conflict at work (Zohar, 1997)

How emotionally or physically disruptive were the following events TODAY?

$0-N / A$

1 - Not Disruptive

2 - Slightly Disruptive

3 - Quite Disruptive

4 - Very Disruptive

1. Had an argument or confrontation about differing views

2. Encountered a lack of cooperation or an inconsideration

3. Had to interact with an inconsiderate or rude person

\section{Daily alcohol use}


1. How many alcoholic drinks did you consume YESTERDAY from 5:00 pm to 11:59 pm [1 drink equals one 12-oz. can or bottle of beer, one 5-oz. glass of wine, one 12-oz. wine cooler or 1 1/2-oz. of liquor straight or in a mixed drink]?

2. Drink day $\mathrm{Y} / \mathrm{N}$

- $\quad D O I F\left(d r \_y 5 L>=1\right)$.

COMPUTE drday=1.

END IF.

EXECUTE.

DO IF (dr_y5L=0).

COMPUTE drday=0.

END IF.

EXECUTE.

VALUE LABELS drday 0 'No' 1 'Yes'.

Instrumental Support (Carver, 1997)

This is a list of some possible ways to deal with stress and negative experiences. Think about the most negative event(s) you experienced in the past 24 hours and please rate the extent to which you used these strategies to cope with this event.

1 - Not at all

2 - A little

3 - Somewhat

4 - A moderate amount

5 - A lot

1. I tried to get advice from someone about what to do

\section{Emotional Support (Carver, 1997)}

This is a list of some possible ways to deal with stress and negative experiences. Think about the most negative event(s) you experienced in the past 24 hours and please rate the extent to which you used these strategies to cope with this event.

1 - Not at all

2 - A little

3 - Somewhat

4 - A moderate amount

5 - A lot

1. I tried to get emotional support from friends or relatives

\section{Demographics}

1. What is your age? (Years) 
2. What is your gender

$$
\begin{aligned}
& 0=\text { Male } \\
& 1=\text { Female }
\end{aligned}
$$

3. Shift worker status

$$
\begin{aligned}
& 1=\text { Regular shift worker } \\
& 2=\text { Shift worker }
\end{aligned}
$$

\section{Day of Week Dummy Codes}

COMPUTE SUn $=0$.

COMPUTE Mon=0.

COMPUTE Wed $=0$.

COMPUTE Thurs $=0$.

COMPUTE Fri=0.

COMPUTE Sat $=0$.

IF (Dayweek=1) Sun=1.

IF (Dayweek=2) Mon=1.

IF (dayweek=4) Wed=1.

IF (dayweek=5) Thurs=1.

IF (dayweek=6) Fri=1.

IF (dayweek=7) Sat=1.

EXECUTE. 